\title{
Plant Growth and LAl Estimation using quantized Embedded Regression models for high throughput phenotyping
}

Dhruv Sheth ( $\sim$ dhruvsheth.linkit@gmail.com )

Ambassador, Edgelmpulse Inc. San Jose, California https://orcid.org/0000-0001-7485-1455

\section{Research Article}

Keywords: Precision Agriculture, Embedded Machine Learning, Embedded Systems, Image Regression

Posted Date: November 9th, 2021

DOI: https://doi.org/10.21203/rs.3.rs-1060088/v1

License: (c) (i) This work is licensed under a Creative Commons Attribution 4.0 International License.

Read Full License 


\title{
Plant Growth and LAI Estimation using quantized Embedded Regression models for high throughput phenotyping
}

\author{
$1^{\text {st }}$ Dhruv. M. Sheth Udayachal High School, Senior Secondary \\ Embedded Machine Learning Ambassador - EdgeImpulse Inc. San Jose. California \\ Mumbai, India \\ dhruvsheth.linkit@gmail.com
}

\begin{abstract}
Phenotyping involves the quantitative assessment of the anatomical, biochemical, and physiological plant traits. Natural plant growth cycles can be extremely slow, hindering the experimental processes of phenotyping. Deep learning offers a great deal of support for automating and addressing key plant phenotyping research issues. Machine learning-based highthroughput phenotyping is a potential solution to the phenotyping bottleneck, promising to accelerate the experimental cycles within phenomic research. The influence of climate change, and due to it's unpredictable nature, majority of agricultural crops have been affected in terms of production and maintenance. Hybrid and cost-effective crops are making their way into the market, but monitoring factors which affect the increase in yield of these crops, and conditions favorable for growth have to be manually monitored and structured to yield high throughput. Farmers are showing transition from traditional means to hydroponic systems for growing annual and perennial crops. These crop arrays possess growth patterns which depend on environmental growth conditions in the hydroponic units. Semi-autonomous systems which monitor these growth may prove to be beneficial, reduce costs and maintenance efforts, and also predict future yield beforehand to get an idea on how the crop would perform. These systems are also effective in understanding crop drools and wilt/diseases from visual systems and traits of plants.Forecasting or predicting the crop yield well ahead of its harvest time would assist the strategists and farmers for taking suitable measures for selling and storage. Accurate prediction of crop development stages plays an important role in crop production management. Such predictions will also support the allied industries for strategizing the logistics of their business. Several means and approaches of predicting and demonstrating crop yields have been developed earlier with changing rate of success, as these don't take into considerations the weather and its characteristics and are mostly empirical.Crop yield estimation is also affected by taking into account a few other factors. Plant diseases enormously affect the agricultural crop production and quality with huge economic losses to the farmers and the country. This in turn increases the market price of crops and food, which increase the purchase burden of customers. Therefore, early identification and diagnosis of plant diseases at every stage of plant life cycle is a very critical approach to protect and increase the crop yield.In this article, I propose an Embedded Machine Learning approach to predicting crop yield and biomass estimation of crops using an Image based Regression approach using EdgeImpulse that runs on Edge system, Sony Spresense, in real time. This utilizes few of the 6 Cortex M4F cores provided in the Sony Spresense board for Image processing, inferencing and predicting a regression output in real time. This system uses Image processing to analyze the plant in a semi-autonomous environment and predict the numerical serial of the biomass allocated to the plant growth.
\end{abstract}

This numerical serial contains a threshold of biomass which is then predicted for the plant. The biomass output is then also processed through a linear regression model to analyze efficacy and compared with the ground truth to identify pattern of growth. The image Regression and linear regression model contribute to an algorithm which is finally used to test and predict biomass for each plant semi-autonomously.

\section{INTRODUCTION}

Advancements in computer vision and machine learning technologies have transformed plant scientists ability to incorporate high-throughput phenotyping into plant breeding. Detailed phenotypic profiles of individual plants can be used to understand plant growth under different growing conditions. As a result, breeders can make rapid progress in plant trait analysis and selection under controlled and semi-controlled conditions, thus accelerating crop improvements. In contrast to existing invasive methods for accurate biomass calculation that rely on plant deconstruction, this system used non-invasive alternative are in commercial applications which leaves the crops intact. Unfortunately, current commercially available platforms are large and very expensive. The upfront investment limits breeders' use of high-throughput phenotyping in modern breeding programs.

For agricultural applications the biomass is a powerful index due to its immediate connection with the crops health condition and growth state. Predicting sequential biomass of plants may serve as important indices to co-relate environmental growth with crop biomass. This approach presents using economical and cost-effective methods to approximate biomass using a Regression approach in Computer Vision DNN models. The regression model uses 2 Dimensional Convolutional Layers. Vision based Regression models help not only in calculating mean difference and increase in biomass but also understand visual cues in plants and predict the biomass evolution based on such cues. The objective of such an approach is to enable temporal analysis of biomass from frames to allow adapting to the planned environment and factors objectively. To take into consideration, wilting leaves observed progressively over frames suggests a decrease in biomass of plant over time, and can be monitored semi autonomously in farms. While existing approaches involve 
unimplementable algorithms or intensive computation, costly hardware or offline or batch processing, which is delayed calculation, this approach attempts to be implementable and not stress on inefficacious data from plants or inference.

Taking One step further to fulfill UN Sustainable Development Goals -

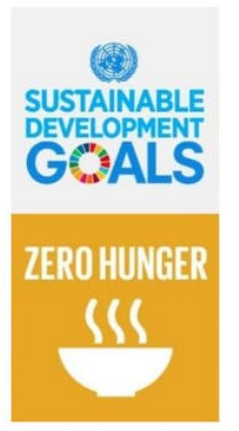

- By 2030 , double the ogricuttural productivity and incomes small-scale food producers, in particular women, indigenous peoples, family farmers, pastoralists and fishers, including through secure and equal access to land, other productive resources and inputs, knowledge, financial services, markets and opportunities for value addition and non-farm employmen

By 2030, ensure sustainable food production systems and implement resilient agricultural practices that increase productivity and production, that help maintain ecosystems, productivity and production, that help maintain ecosystems. that strengthen capacity for adaptation to climate change, extreme weather, drought, flooding and other disasters and that progressively improve land and soll quality

SONY

Fig. 1. Explaining UN's SDG's and how this project is bringing it one step closer to life

This project aims to expand scope of UN's second SDG and entail few of the Goal Targets by bringing in semi-autonomous monitoring systems for food production monitoring and yield production methodology to "increase productivity and production by implementing resilient agricultural practice." as highlighted in second UN SDG goal target.

A.

1) :

a) Material and Methods::

Data Accumulation :: Most of the dataset used to train this model was adopted from the Paper "Growth monitoring of greenhouse lettuce" by Lingxian Zhang et al. There were 3 kinds of datasets offered in this paper, one of them being the raw dataset curated under unmonitored sunlight conditions. The other dataset was an augmented version of the raw dataset synthesized and generated with all images having similar light, illuminance and saturation. The third dataset contains spatial and depth information of these plants under the same environment and observed growth patterns. In this approach, we'll be using the augmented data set to increase efficacy of model and couple images in a similar visual pattern.
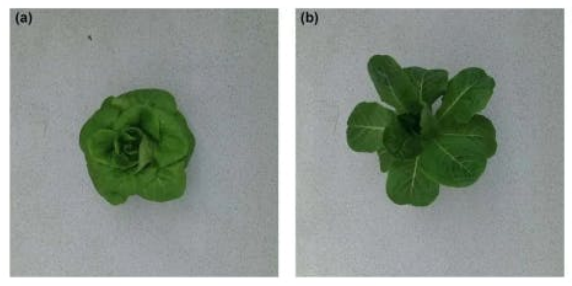

Fig. 2. (Left) Lettuce Flandria Cultivar. (Right) Lettuce Tiberius Cultivar. source - [Growth monitoring of greenhouse lettuce paper]

Greenhouse lettuce image collection and preprocessing -: The experiment was conducted at the experimental greenhouse of the Institute of Environment and Sustainable Development in Agriculture,

Chinese Academy of Agricultural Sciences, Beijing, China $\left(\mathrm{N} 39^{\circ} 57^{\prime}, \mathrm{E} 116^{\circ} 19^{\prime}\right)$. Three cultivars of greenhouse lettuce, i.e., Flandria, Tiberius, and Locarno, were grown under controlled climate conditions with $29 / 24{ }^{\circ} \mathrm{C}$ day/night temperatures and an average relative humidity of $58 \%$. During the experiment, natural light was used for illumination, and a nutrient solution was circulated twice a day. The experiment was performed from April 22, 2019, to June 1, 2019. Six shelves were adopted in the experiment. Each shelf had a size of $3.48 \times 0.6 \mathrm{~m}$, and each lettuce cultivar occupied two shelves. [4]

The number of plants for each lettuce cultivar was 96, which were sequentially labeled. Image collection was performed using a low-cost Kinect 2.0 depth sensor. During the image collection, the sensor was mounted on a tripod at a distance of $78 \mathrm{~cm}$ to the ground and was oriented vertically downwards over the lettuce canopy to capture digital images and depth images. The original pixel resolutions of the digital images and depth images were $1920 \times 1080$ and $512 \times 424$, respectively. The digital images were stored in JPG format, while the depth images were stored in PNG format. The image collection was performed seven times 1 week after transplanting between 9:00 a.m. and 12:00 a.m. Finally, two image datasets were constructed, i.e., a digital image dataset containing 286 digital images and a depth image dataset containing 286 depth images. The number of digital images for Flandria, Tiberius, and Locarno was 96, 94 (two plants did not survive), and 96, respectively, and the number of depth images for the three cultivars was the same.

Since the original digital images of greenhouse lettuce contained an excess of background pixels, this study manually cropped images to eliminate the extra background pixels, after which images were uniformly adjusted to $900 \times 900$ pixel resolution.The Figure below shows examples of the cropped digital images for the three cultivars.

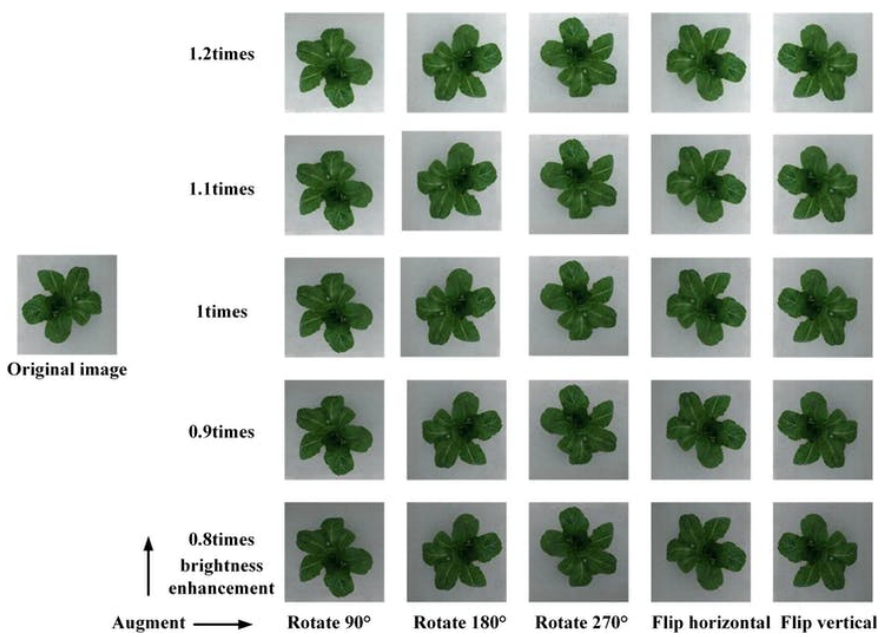

Fig. 3. source - [Growth Monitoring of Greenhouse Lettuce] Lingxian Zhang et al.

Prior to the construction of the CNN model, the original digital image dataset was divided into two datasets in a 
ratio of $8: 2$, i.e., a training dataset and a test dataset. The two datasets both covered all three cultivars and sampling intervals. The number of images for the training dataset was 229 , where $20 \%$ of the images were randomly selected for the validation dataset. The test dataset contained 57 digital images. To enhance data diversity and prevent overfitting, a data augmentation method was used to enlarge the training dataset.. The augmentations were as follows: first, the images were rotated by $90^{\circ}, 180^{\circ}$, and $270^{\circ}$, and then flipped horizontally and vertically. To adapt the CNN model to the changing illumination of the greenhouse, the images in the training dataset were converted to the HSV color space, and the brightness of the images was adjusted by changing the $\mathrm{V}$ channel. The brightness of the images was adjusted to $0.8,0.9$, 1.1 , and 1.2 times that of the original images to simulate the change in daylight. [4]

The raw dataset acquired was thus augmented and optimized to be fed into the Convolutional Neural Network for Regressional analysis. These aligned image pairs serve as input dataset in EdgeImpulse Studio. The below Figure illustrates how raw images perform as compared to Augmented ones with equalized lighting and saturation throughout the images.

\section{(Lettuce Flandria)}

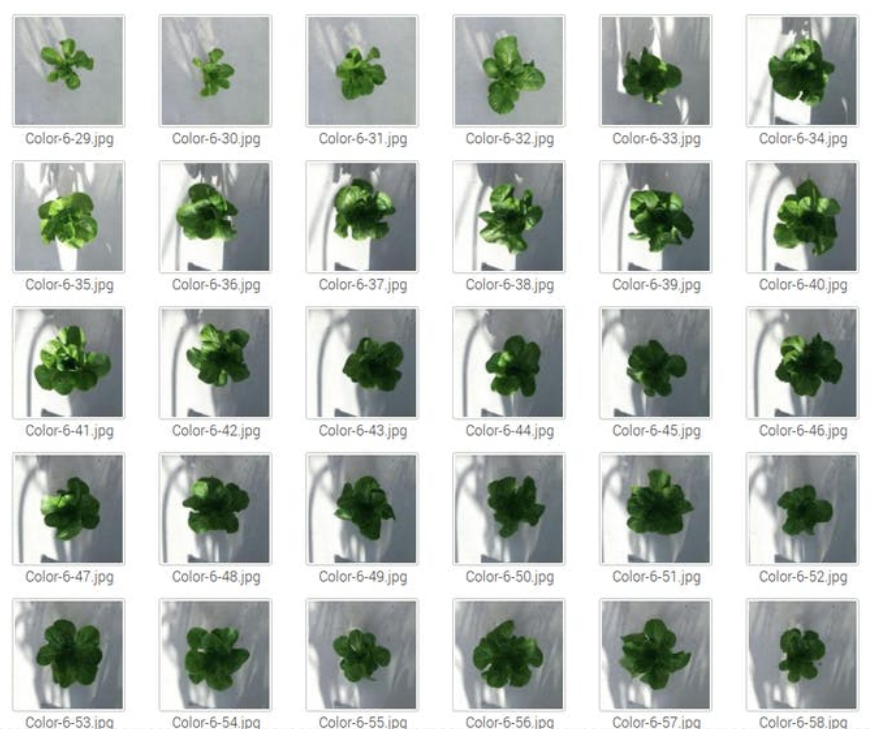

Fig. 4. Illustrates how raw images show varying illuminance which might be a major drawback for the neural network.

Figure given below Illustrates Lettuce Tiberius Dataset-

The above figure demonstrates Synthesized Images for Neural Network possessing equalized illuminance, RGB Channels and saturation to maintain consistent Data input for CNNs.

The synthesized images were further augmented and a translational blur parameter of 0.01 was added to the images to be able to predict the biomass of lettuce even while the source from which the image is being captured is in motion. An example of translational motion blur are noticed on motorized plates or Machine Motion Drivers illustrated below -

The figure shown below demonstrates how the data for the Lettuce variants are captured and ingested. The camera angles were placed vertically perpendicular with respect to the ground

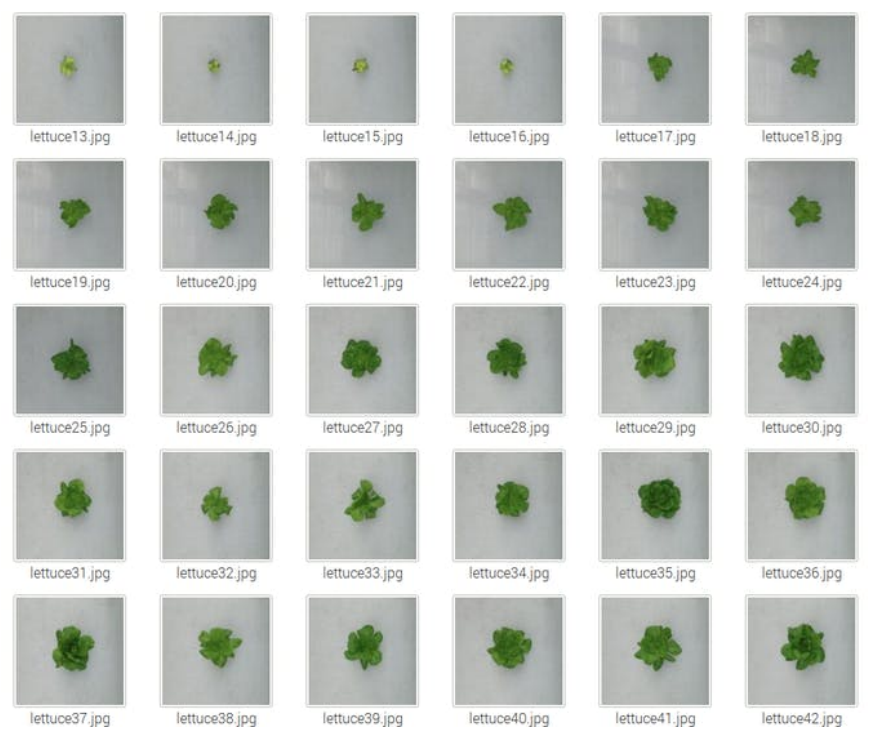

Fig. 5. Fig - Synthesized Images for Neural Network possessing equalized illuminance, RGB Channels and saturation to maintain consistent Data input for Neural Network.

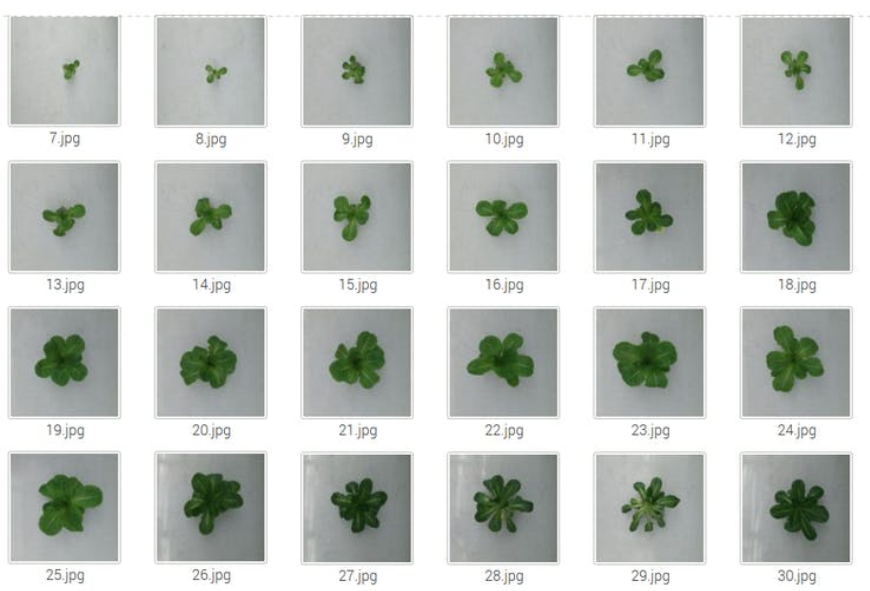

Fig. 6. Lettuce Tiberius dataset used for training model specific to test on Tiberius plant subtype.
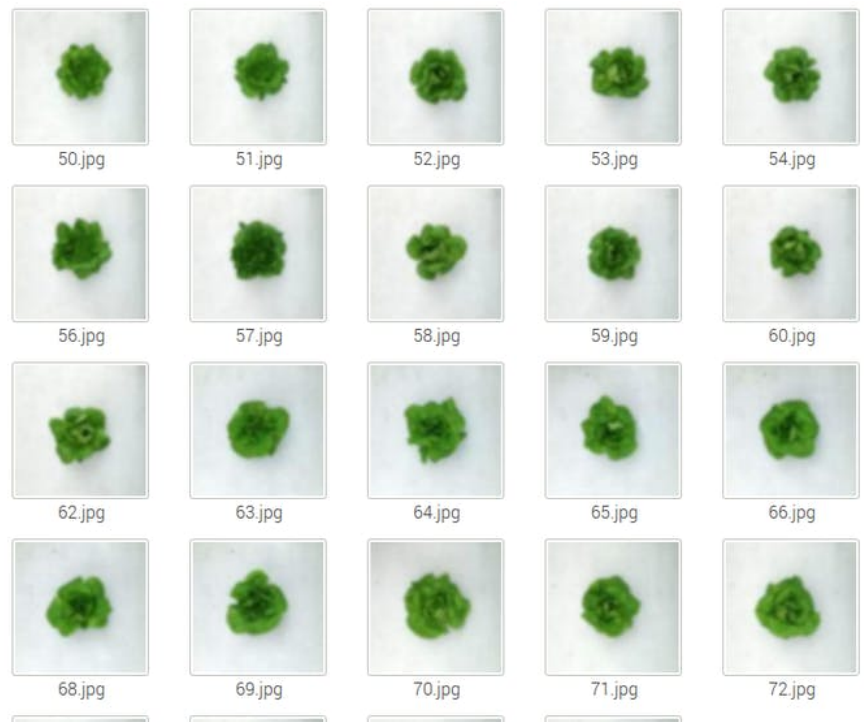

Fig. 7. A translational motion blur of 0.01 added to synthesized dataset. 


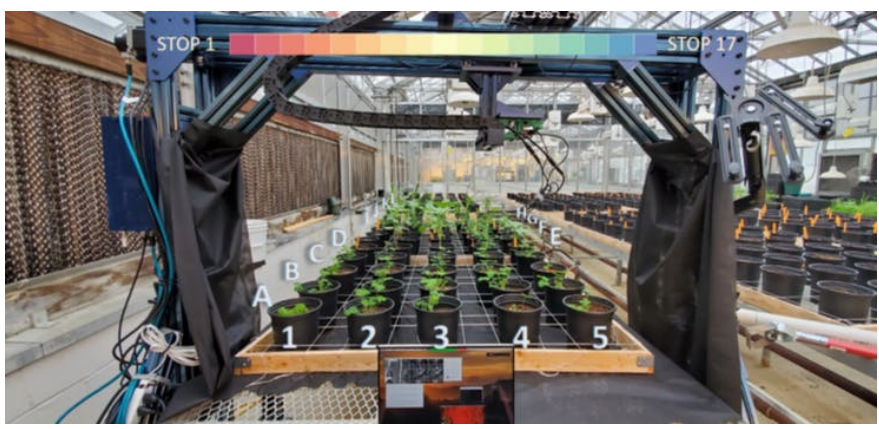

Fig. 8. Translational Data Collection from motorized plates. Source - Paula Ramos Et Al. Precision Sustainable Agriculture

plane and the distance between ground plane was adjusted to $78 \mathrm{~cm}$ or $0.78 \mathrm{~m}$ for each plant to ensure standardized images and ensure deviation in biomass increase and Leaf growth is in constant incrementation. The approximate image area covered by each cultivar is 4.176 meter squared and the approximate image area covered by each lettuce shoot is 0.0435 meter squared. The images captured are standardized to $128 * 128$ pixels to make it easier for DNNs to scale and process. This implies that each $128 * 128$ pixel image occupies 0.0435 meter squared or $435 \mathrm{~cm}$ squared area. Each pixel would hence occupy $2.65 \times 10^{\wedge}-2 \mathrm{~cm}$ squared area. This conversion to ground scale is essential for computing not only relative but also absolute Leaf Area Index and Biomass for each plant predicted and verify it with ground truth. The process below has to be imitated while capturing test images or if the laboratory conditions vary, then adjust Field of Object in the image frame and either zoom in or zoom out corresponding to distance between/ depth of camera and plant.
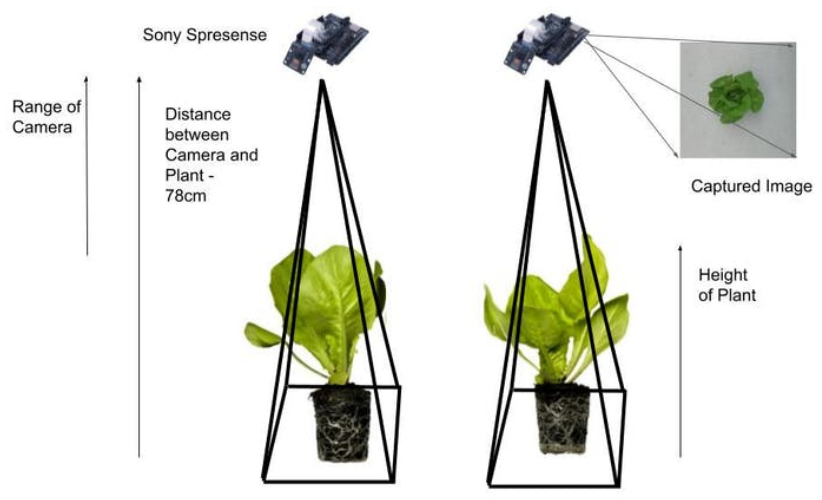

Fig. 9. Field of Object in Image frame and image capturing process illustrated

\section{Embedded Inference Board used for Real time process- ing:}

The Sony Spresense Neural Inference board, main board and Camera system:

The Sony Spresense is a suite of Embedded systems mainly suited and widely used for Vision based solutions consisting image classification, inferencing and regression based prediction. It is ideally suited for low power, real time inferencing applications, suited for this system. The Sony Spresense comes with on-board $1536 \mathrm{kB}$ RAM and $8192 \mathrm{kB}$ ROM for Infer- ence. The system allows processing multiple pipelines and DNNs/CNNs withing the data range. The main board itself is compact enough to be dropped into many production-grade systems without much fuss, and from a software standpoint, there are several options available, from using $\mathrm{C} / \mathrm{C}++\mathrm{SDK}$ or Python API, whichever goes best with the system. This application features using pre-compiled $\mathrm{C}++$ binaries for the system from EdgeImpulse Studio, leaving compilation headaches to the EdgeImpulse compiling studio.

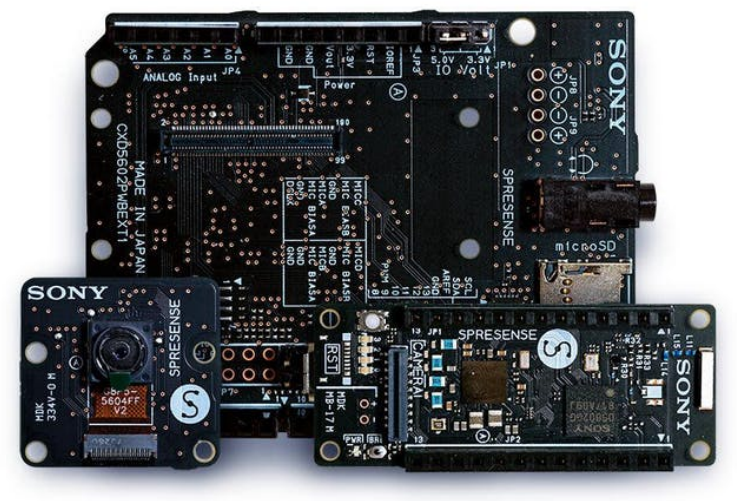

Fig. 10. The Sony Spresense suite featuring a main board, camera shield and neural processing board

The proceeding diagram demonstrates test data accumulation and live classification setup using Sony Spresense which will be elaborated in the latter part.

Sony Spresense Camera setup for collection and test on on Deployed Regression Model

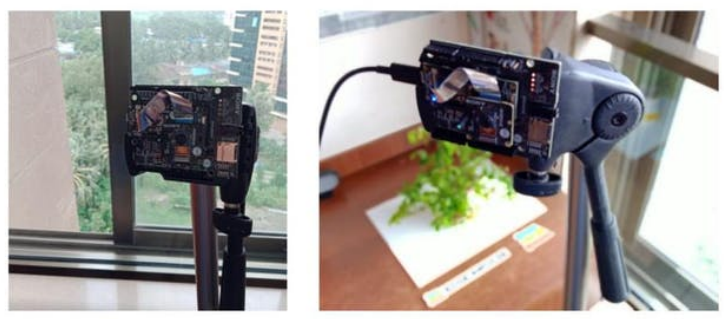

Fig. 11. Live Classification and data accumulation process for the regression model

\section{Pre-Processing and Feature Extraction:}

A. Ingesting the Cultivar Dataset to EdgeImpulse Studio for pre-processing and Feature Extraction:

For starters, EdgeImpulse has provided more details on how to get started with EdgeImpulse in their docs section - Getting Started

There are two methods of data upload on the EdgeImpulse Studio, one of them including the EdgeImpulse Ingestion uploader API - https://docs.edgeimpulse.com/referenceuploader API and the other being the visual uploader which in most 
cases is more preferred, for ease and simplicity. The figure demonstrates visual data ingestion process.

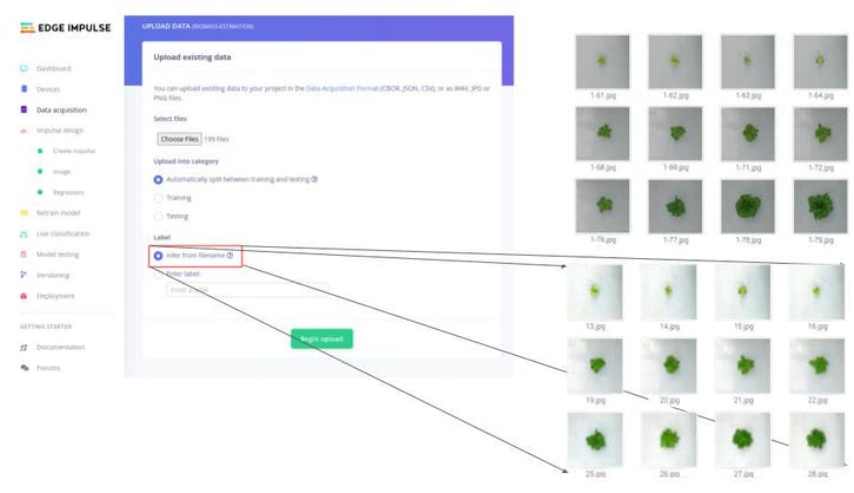

Fig. 12. EdgeImpulse Dashboard Data ingestion process

Here, the infer from filename selection is used for creating a regression dataset. Regression dataset classes are numerically named in progression of images to be fed in the CNN. The $\mathrm{CNN}$ uses numerical class interpolation instead of class name to train the regression model.

The dashboard sorts and orders data and is displayed with number of classes, image signature and label and a few filters to sort dataset.

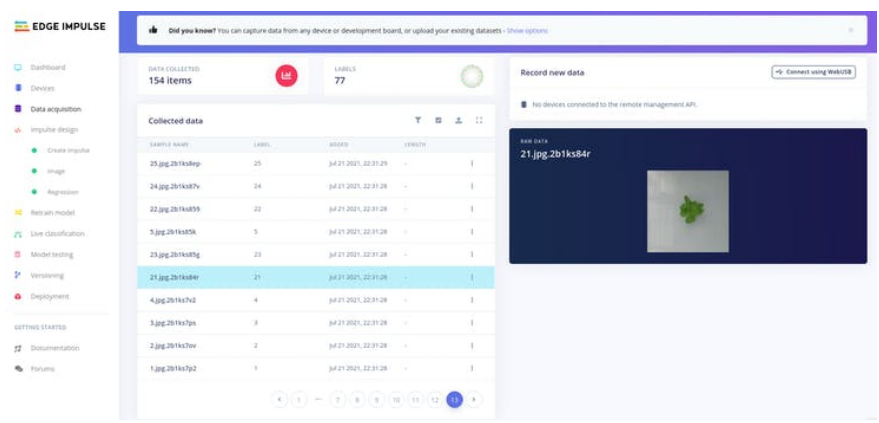

Fig. 13. EdgeImpulse Dashboard after the dataset is ingested.

Thereafter, an impulse is created in the impulse design tab with the necessary input block, processing block, learning block and output features.

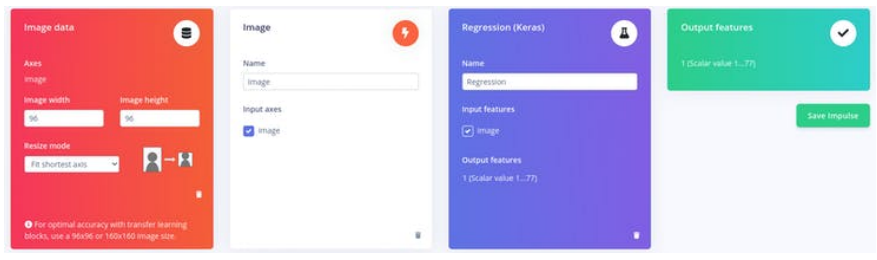

Fig. 14. Creating and Impulse, designing CNN pipeline.

The parameters block takes in pixel-by-pixel input features and converts them to scalar Raw features which can be interpreted by the CNN.

For each image processed, it consists of a feature and EdgeImpulse Studio projects a plot of these features in terms of 3 Visualization layers. A local visualization of the learned

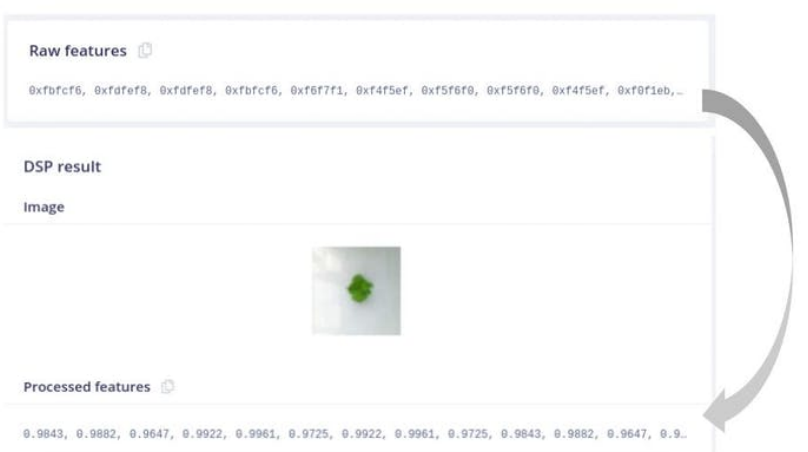

Fig. 15. Processing Raw features as processed features

features is plotted which explains the pixels important for classification.

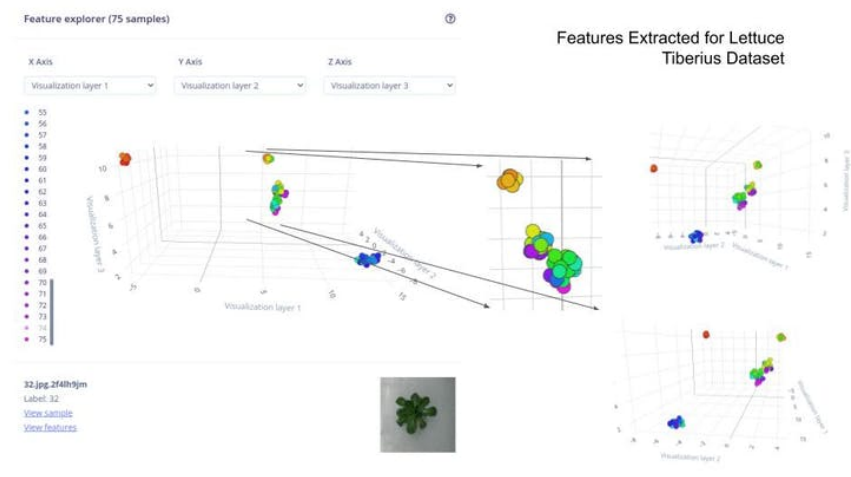

Fig. 16. Features Extracted for Lettuce Tiberius Dataset

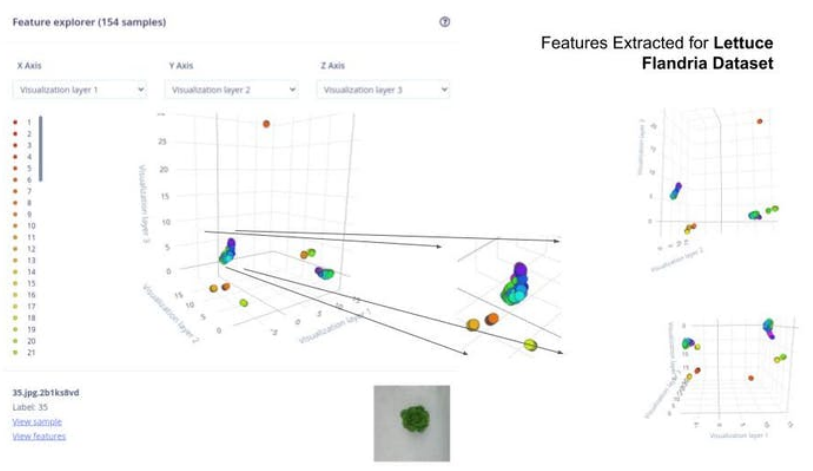

Fig. 17. Pre-processing and Feature Extraction of Lettuce Flandria Dataset

\section{Construction of the CNN and Performance EVALUATION:}

The above image illustrates the Architecture of the CNN Model. It consists of two 2D Convolutional Layers, two pooling layers and one Fully Connected Layer (FCN).

The CNN took pre-processed, 3 Channel images of Greenhouse Lettuce of size 128x128 after the feature-extraction was completed. Each convolution layers was capped with Kernels of size $3 \times 3$ which were used to extract the features. The Max 


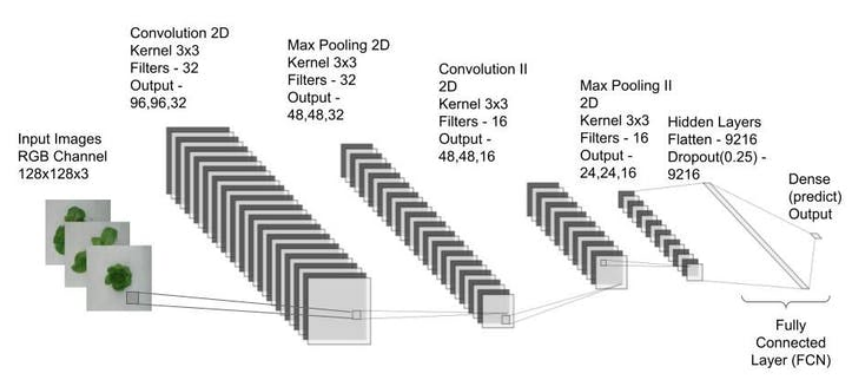

SONY

Fig. 18. Representation of the CNN Architecture used for Training the Regression Model

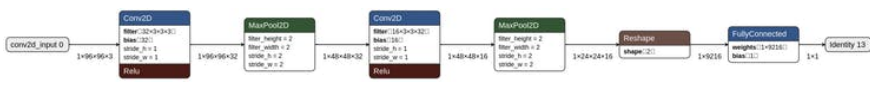

Fig. 19. Netron Neural Network Render of the CNN Architecture

Pooling Layers in the CNN were adapted with Kernels of size $2 \times 2$ and stride of 2, which is set to default in most CNNs. The CNNs were equipped with Max Pooling Function instead of an average polling function. A Dropout of rate 0.25 was used in the CNN to stabilize the rate of Dropout and not exceed to a higher rate of 0.5. A constant Learning Rate of 0.002 was set in the model, and the Batch Size was reduced to 16, which performed marginally better in comparison to 32 . The Neural Network was trained and capped at 225 epochs, after which the loss function started peaking again. Mean Squared Error function was used to evaluate the loss function in the model. A few other hyperparameters were adjusted and evaluated to increase the model efficacy.

EdgeImpulse Studio provides default CNN Architectures selected for the Regression Model which are smaller and allow real time performance. If you wish to edit few of the Architectural layers, you can do so in the EdgeImpulse Studio.

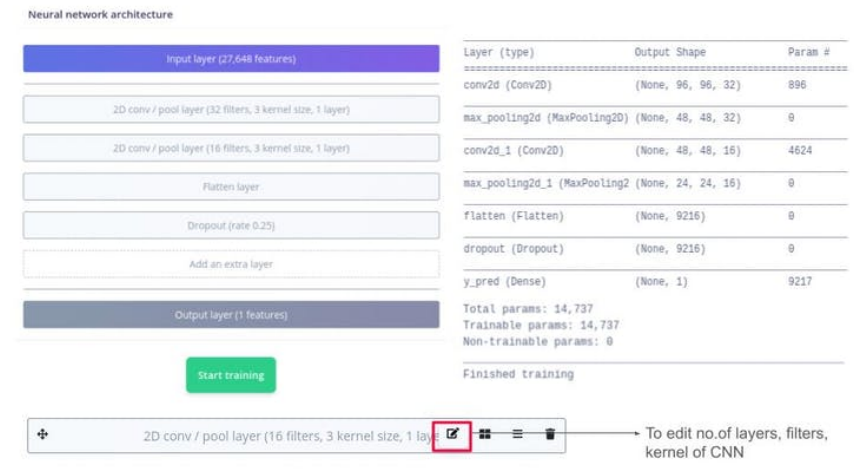

Fig. 20. Flexible Functionality to alter the architecture of the CNN model in EI Studio.

\section{LOSS RATES AND ACCURACY:}

Two models were subsequently trained to compare performance and loss rates between change in parameters used for

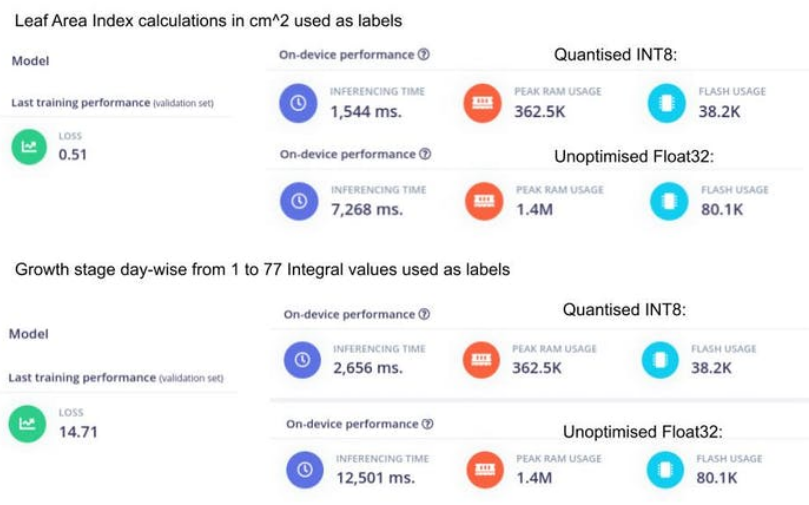

Fig. 21. Comparing Change in model performance with labels set as biomass values, in comparison with (down) Day wise labels of growth stage of plant

labels. For the first model, the actual Leaf Area Index(LAI) (Ground Truth) Calculated via segmentation of the plant was used as labels. These are rational, decimal numbers like $77.8135,90.4532$ in $\mathrm{cm}^{\wedge} 2$ units.

The second model included Day-wise labels corresponding to growth stages of the plant. Eg- for data captured on Day 1 - label 1 was used (unitless integral vales). The second model did not show complete consistency in plant growth progression and hence the model performed poor and had larger weights and features to learn from.

To my surprise, the model which contained labels in terms of LAI achieved near stellar accuracy of 0.51(MSE - Mean Squared Error), and the second model was much heavier, slow in inference and also had a high loss function of 14.71.

The Regression model performed significantly better as compared to usual Regression models which peak at a loss rate of 100. The loss function is calculated using Mean Squared Error gradient. The Epochs were set to 130 for training, while the learning rate to 0.005 , which allowed faster learning and better results. The model loss stabilized after 35 epochs after which it continuously converged to a plateau descent, and remained stable for the rest. Comparing the int8 quantised model which edges at 0.51 loss rate at par with unoptimised float32 model, the quantised model performs much better when deployed on the Sony Spresense. The float 32 model carries an inference time of $7.268 \mathrm{~s}$ per frame which is definitely not suited for real time classification. Comparatively, the int8 model outweighs with $1.544 \mathrm{~s}$ per frame, $362.5 \mathrm{~K}$ memory usage and $38.2 \mathrm{~K}$ Flash.

For the following research, a lot of data analysis and feature engineering has been done for the data ingested into the EdgeImpulse Studio. The following plot is an example of this. While comparing the model performance, this is the relation between the labels of model 1 ( $\mathrm{x}$-axis ) to labels of model 2 ( $y$-axis ). The linear plot is not completely linear, and hence there is a deviation in results. Data analysis of the segmented LAI helped in finding out model efficacy in this example.

\section{Data Analysis and Adaptive Thresholding:}

The Leaf Area Index or Biomass for each plant was calculated using image segmentation which was achieved by 


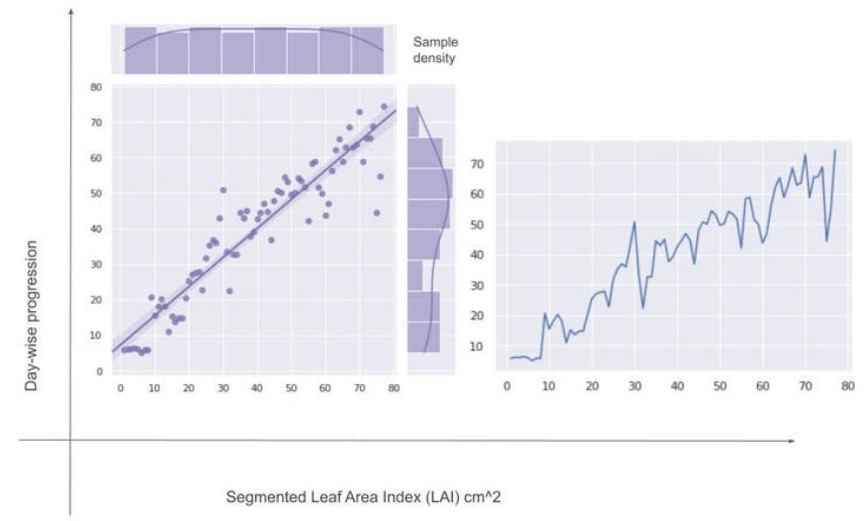

Fig. 22. Relplot using Seaborn to analyze inter-relation between LAI labels and Day-wise progression labels

using the adaptive threshold method for the color information, specifically the Otsu Threshold, followed by a floodfill algorithm in OpenCV and finally using pixel-by-pixel segmented area calculation methods. This pipeline is illustrated below:

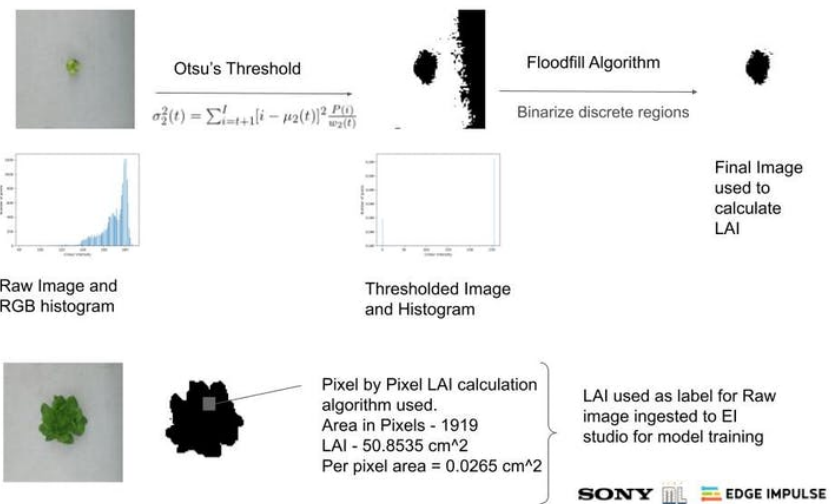

Fig. 23. Pipeline created to estimate Ground Truth LAI

The above figure demonstrates the pipeline created by me to process and output Ground Truth LAI using Thresholding method for segmentation. The LAI (Leaf Area Index ) calculated corresponding to the raw image is later used as label for the image and ingested to the EdgeImpulse Studio. The pipeline used in the above process is as follows. An adaptive thresholding mechanism known as Otsu's threshold is used to segment the image from the contrastive background. This is comparatively easy due to the color contrast between the object i.e the plant and the background. However, for instances where the LAI is less than $10 \mathrm{~cm}^{2}$, the Otsu threshold segments the image leaving some noise at the periphery of the confined region. This hampers the overall LAI estimation. Hence, for these images where the plant area is $\ll<$ average area, a Floodfill algorithm is used to binarize the noise or holes in image and allow smoother LAI calculation.

This is the defined pipeline for all the samples in the cultivar accumulated, and the process of calculation of LAI for Ground Truth Samples. Post floodfill algorithm being applied, a pixel by pixel area calculation function is applied
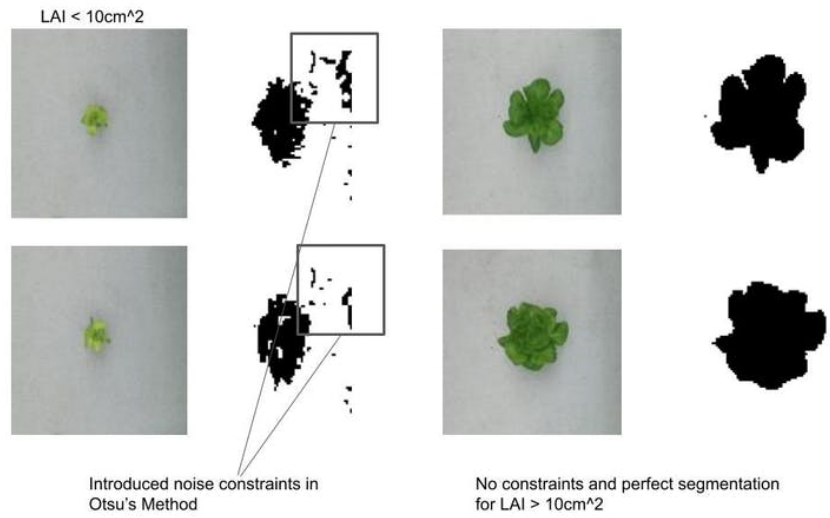

Fig. 24. Comparing Otsu's threshold in Samples with LAI $<$ or $>10 \mathrm{~cm}^{2}$

on binarized images using numpy. The area is calculated in pixels, and using a transformation formula mentioned in the data collection topic, the area in pixels is transformed to LAI.

$$
\begin{aligned}
& L A I_{(\text {perplant })}=[[2 *(3.48 * 0.6 \text { metre }) / 100 n] * \text { pixels }] \mathrm{cm}^{2} \\
& \mathrm{n}=\text { number of plants in cultivar } \\
& \text { pixels }=\text { Area in pixels }
\end{aligned}
$$

Fig. 25. formulating LAI Index measure caclulation per pixel

The formula can be reduced to $2.605 \times 10^{-2} \times$ Area in pixels $\mathrm{cm}^{2}$. This formula is only confined when the distance between the lettuce cultivar and camera i.e sony spresense is $78 \mathrm{~cm}$.

The final results for Otsu segmentation for all plants in cultivar is as follows

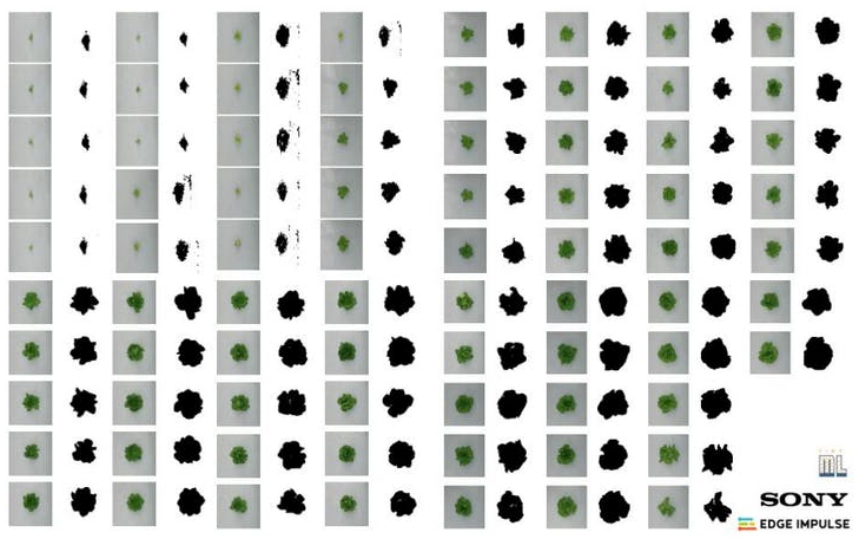

Fig. 26. Adaptive Thresholding segmentation conducted on cultivar of 77 samples

The Adaptive Thresholding procedure was conducted on a cultivar of Lettuce Flandria plants and used as Ground Truth labels for ingesting the Dataset. A more elaborate view of segmented images per 20 samples is given below:

The python script used for segmentation procedure and adaptive thresholding will be provided in the github repository attached with the code. The analysis of this data in a seaborn plot had been performed above comparing the label numerical 


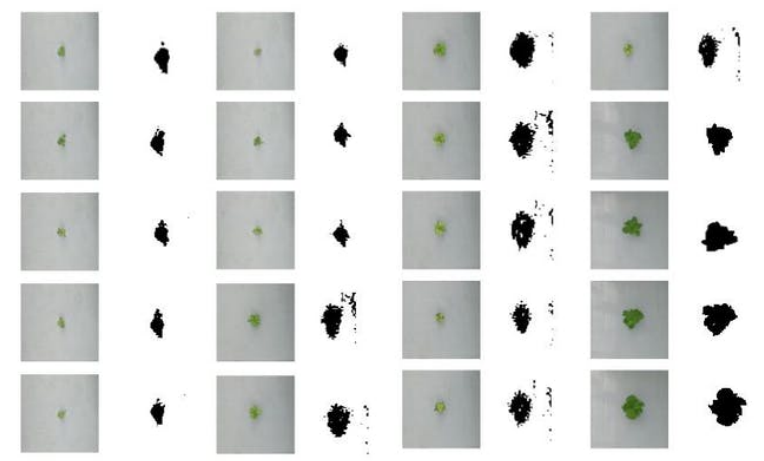

Fig. 27. Thresholded Cultivars from Day 1 to 20

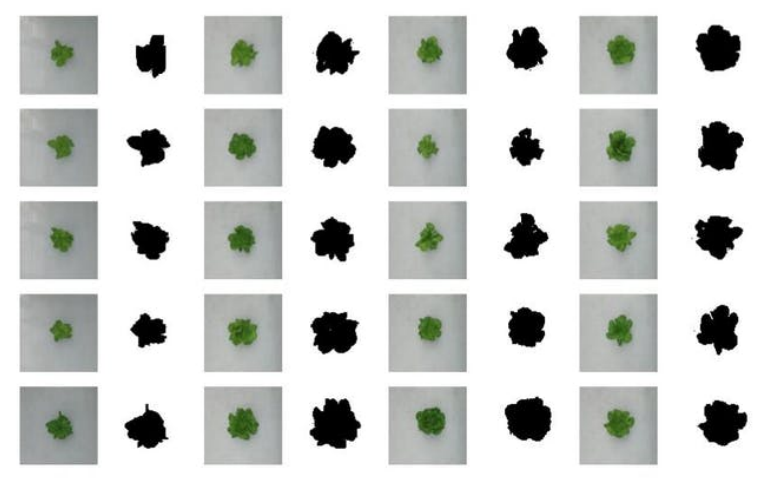

Fig. 28. Thresholded Cultivars from Day 21 to 40

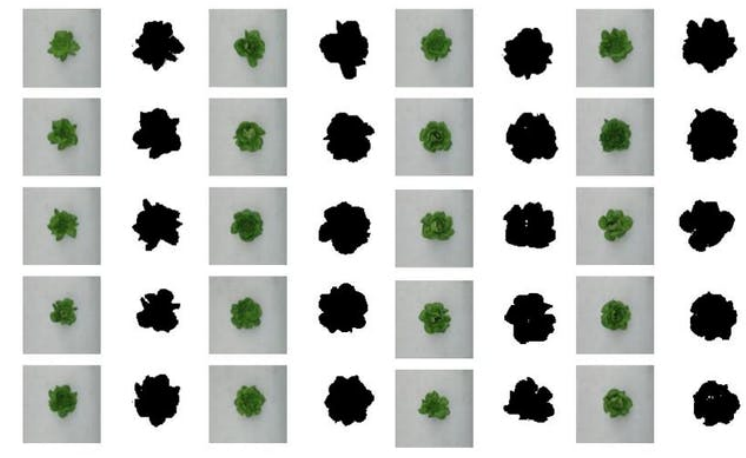

Fig. 29. Thresholded Cultivars from Day 41 to 60

value extracted using LAI adaptive thresholding with Day-wise progression. Refer that plot to see complete data analysis of the above segmented pixel by pixel LAI values.

\section{Vi. Model Testing and Evaluation:}

After the model training and data analysis of the segmented labels has been completed, the values predicted from the Regression model trained in EdgeImpulse Studio can be tested to evaluate efficacy on unseen test data. The model

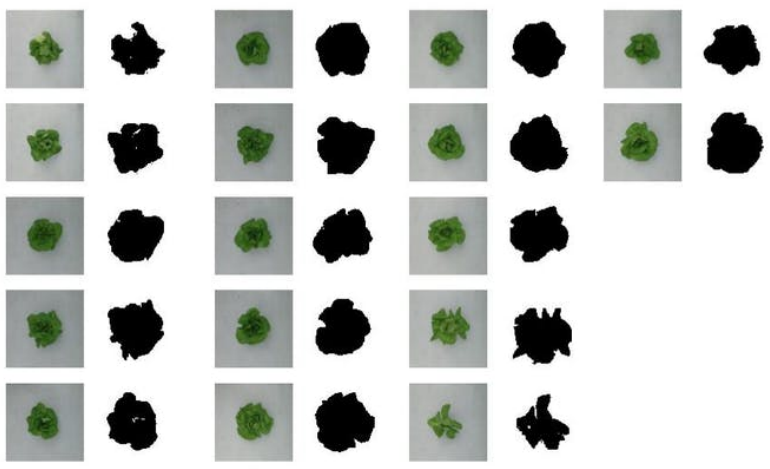

Fig. 30. Thresholded Cultivars from Day 61 to 80

performs with near stellar accuracy on testing data evaluation in EdgeImpulse Studio. A test dataset of 19 samples with unique values from $5 \mathrm{~cm}^{2}$ to $90 \mathrm{~cm}^{2}$. LAI is fed to the model. The model evaluates the image data without the input of labels and proposes it's predictions. The predictions within a mean deviation of around $5 \mathrm{~cm}^{2}$. The maximum deviation or error rate withing limited cluster where it's predicted accurate is 4.78. On an average Lettuce Flandria observed much better performance than Lettuce Tiberius. The RMSE calculated for Lettuce Flandria was found out to be $1.185954306 \mathrm{~cm}^{2}$. The RMSE is calculated using the metrices described below:

$$
\mathrm{RMSD}=\sqrt{\frac{\sum_{i=1}^{N}\left(x_{i}-\hat{x}_{i}\right)^{2}}{N}}
$$

$$
\begin{array}{ll}
\text { RMSD } & =\text { root-mean-square deviation } \\
i & =\text { variable } \mathrm{i} \\
N & =\text { number of non-missing data points } \\
x_{i} & =\text { actual observations time series } \\
\hat{x}_{i} & =\text { estimated time series }
\end{array}
$$

Fig. 31. Formula used to calculate LAI RMSE on predicted outcome

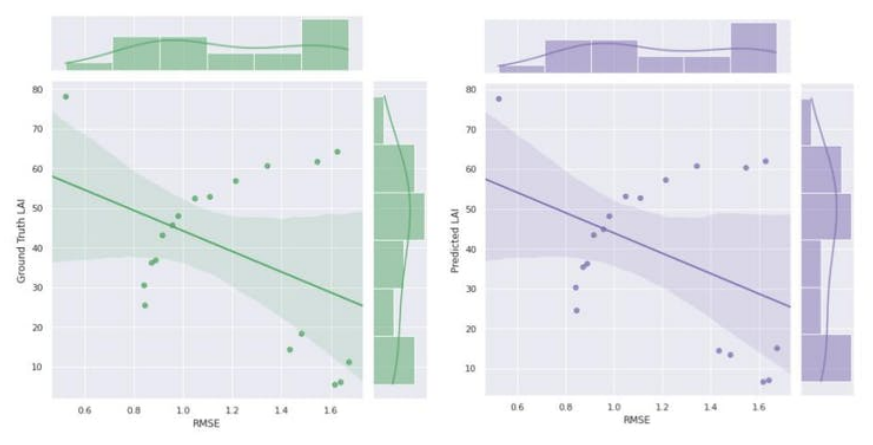

RMSE compared to Ground Truth LAI (Left) and Predicted LAI (Right)

Fig. 32. RMSE Compared to Ground LAI (Left) and Predicted LAI (Right)

The above plot, plotted using seaborn demonstrates a com- 
parative analysis between Ground Truth LAI and RMSE and Predicted LAI and RMSE. The plot explains how the RMSE increases with increase in LAI, and a few anomalies are found in that variation. Overall, its a graph with decreasing slope, and exponentially increasing RMSE with increasing LAI.

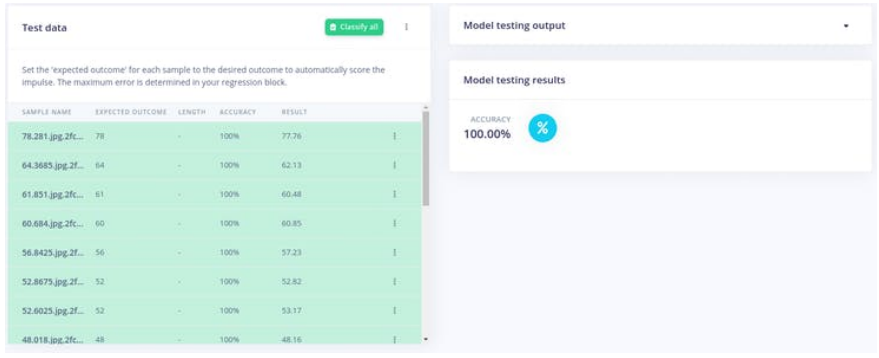

Fig. 33. Prediction summary of over 19 samples in test dataset

The above images are captured from Testing tab in EdgeImpulse Studio. The ingested Test Dataset achieve 100 percentage accuracy which might seem superficial, but it is evaluated on 19 samples with loss of 0.51 which makes is obvious that the model performs exceptionally well! The input Ground Truth Data and predicted Data of LAI by EdgeImpulse Regression model is summarized in the plot following the paragraph:
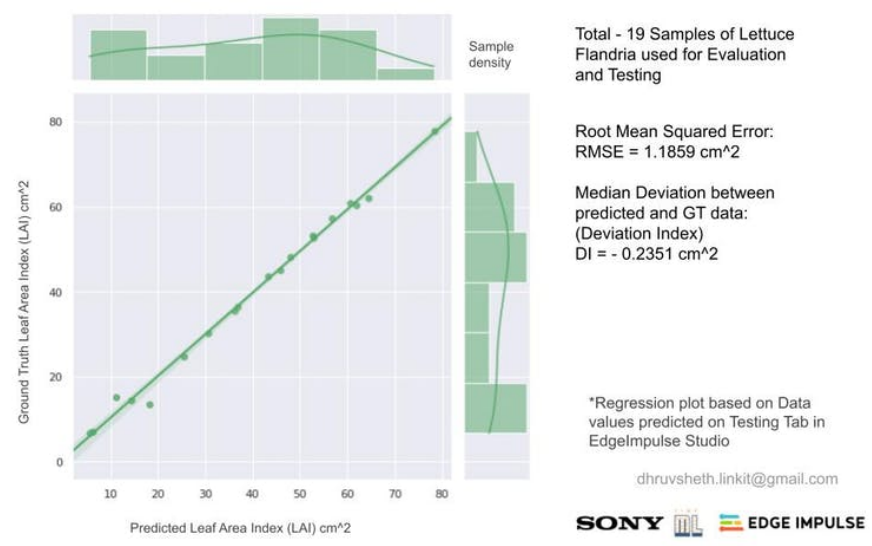

Fig. 34. Plot comparing Ground Truth and predicted LAI values from EdgeImpulse Studio Model Testing section

The plot compares how Predicted LAI performs as compared to Ground Truth LAI for a confined segmented sample. The regression model trained in EI Studio performs and produces accurate predictions for almost all samples. There is an increased error rate in the region between $15-20 \mathrm{~cm}^{2}$ LAI Ground Truth labels, which indicates the increase in noise in data segmentation in Otsu's segmented images. The Regression model, predicts LAI lower than expected, due to noise in threshold samples, which results in an increase in LAI than expected. The Average RMSE was found to be 1.859 $\mathrm{cm}^{2}$ which is an indicative factor of accurate predictions. In RMSE, the function, $\mathrm{X}_{o}-\mathrm{X}_{i}$ which is a difference between observed and predicted data. This index was also found out among the data samples predicted and it averaged at -0.2351 $\mathrm{cm}^{2}$, indicating that the predicted data is on an average 0.235 $\mathrm{cm}^{2}$ less than Ground Truth.

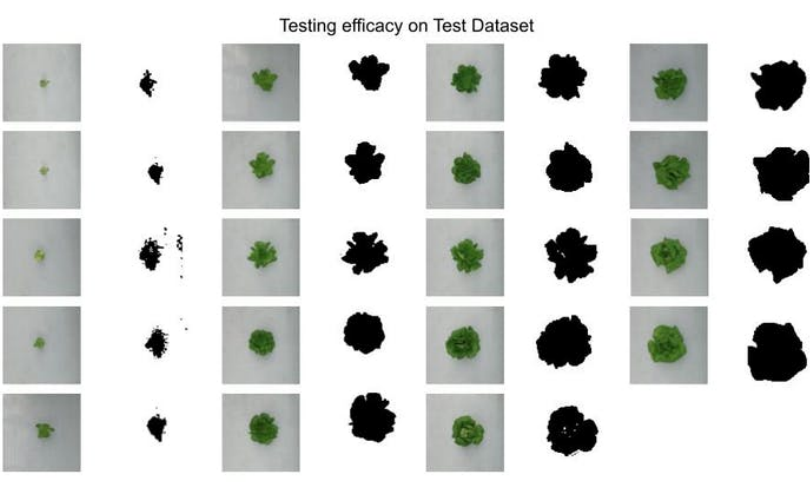

Fig. 35. LAI of segmented images

Above figure represents LAI of segmented images used as test data set to test efficacy. As observed above, the results showed strong correlations between Ground Truth Segmented data and from those predicted by the CNN model. These correlations are measurements concluded from input test images rather than scalar/numerical input data, which makes a huge difference in how effectively CNNs for computer vision models have developed, and become light-weight with increased efficacy without straining on model performance. The quantization of these models done by EdgeImpulse Studio is a massive plus point in Embedded Machine Learning systems most importantly with low power, real time inference in Computer Vision systems.

\section{Deploying Model to Sony SPRESEnSE ANd REAL World DATA TeSTING:}

EdgeImpulse offers a unique compilation system for Embedded ML models which help in quantization of models for upto 55\% less RAM! and 35\% less ROM! while maintaining consistent accuracy and loss scores. This is a feature I adore about the EdgeImpulse Studio. In the deployment section of EdgeImpulse Studio, there are a list of pre-compiles binaries for supported boards or libraries which can be self-compiled.This project utilizes the Sony Spresense PreCompiled Binary which can be directly deployed on the board for real time inference.

With the EON compiler, there is a significant reduction in RAM usage on-board as well as the ROM usage. The RAM usage decreases from $435.6 \mathrm{~K}$ to $362.5 \mathrm{~K}$, nearly $17 \%$ reduction in RAM usage, and from $53.5 \mathrm{~K}$ to $38.2 \mathrm{~K}$ decrease in ROM/Flash usage, $29 \%$ reduction in ROM usage. With the EON Compiler enabled, build the model and flash it over Sony Spresense board.

A complete log of compilation and build process can be found at "Build output". Sony Spresense observes real time inference and result estimation on board in under $1 \mathrm{~s}$, to be precise, nearly $922 \mathrm{~ms}$ !

\section{ViII. Conclusion:}

Demonstrating Low Power Consumption and battery operated remote system: 


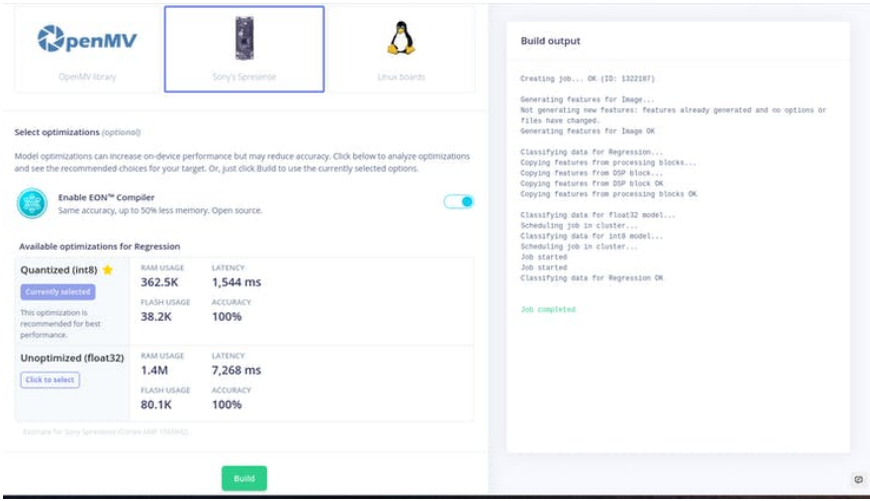

Fig. 36. Compiled quantised binary for Regression model on Sony's Spresense

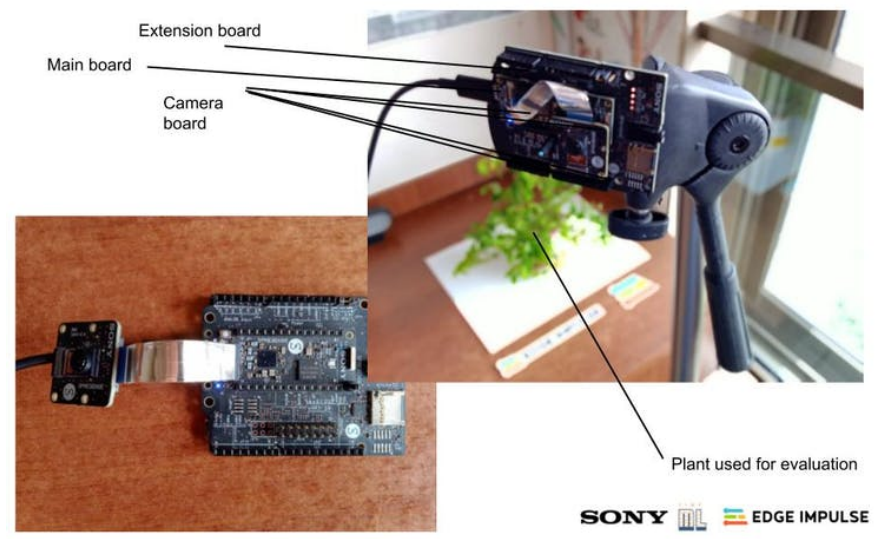

Fig. 37. Demonstrating various components used in the hardware system for Test Data Acquisition

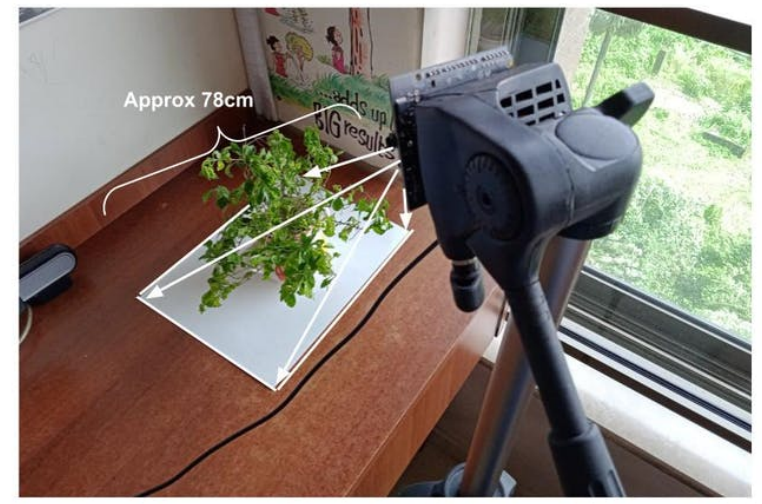

Fig. 38. Demonstrates distance between Camera and Plant for the model to work
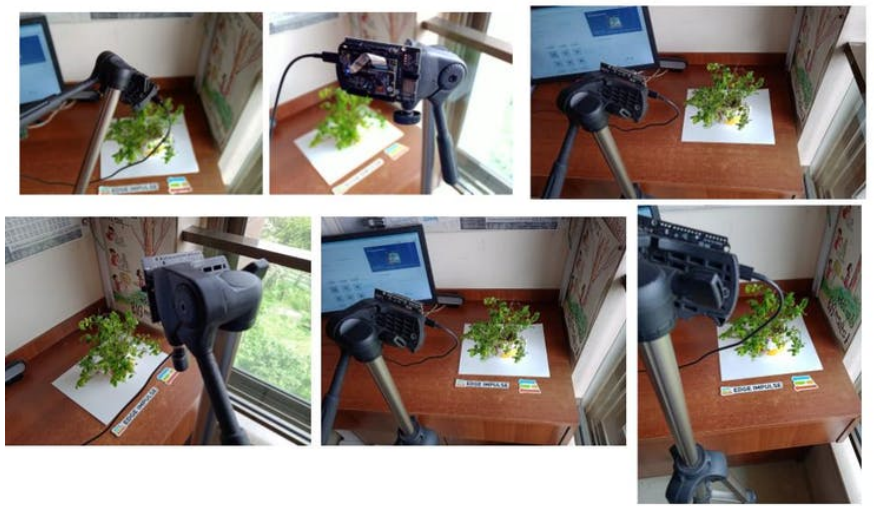

Fig. 39. Various images of live classification and on-board processing algorithms

The preceeding images demonstrate the live classification system and real time inference on the Sony Spresense board. The board acquired images from over the plant, inferences the data, processes it and predicts a suitable LAI outcome in real time. The illustrations in images explain the structure of the system, approximate distance and data acquisition procedure for real time on-board inference. The approximate power usage on board is $0.35 \mathrm{~A}$ per hour which is easily powered by a battery system, here as a powerbank. The tested system lasts for 20.5 hours effortlessly over a single charged powerbank. If the clock frequency of the board is set to $32 \mathrm{MHz}$, the average power consumption reduces significantly. The complete system, while in production is expected to be completely battery operated over a suitable voltage power bank, more preferably 1A, which I have used here

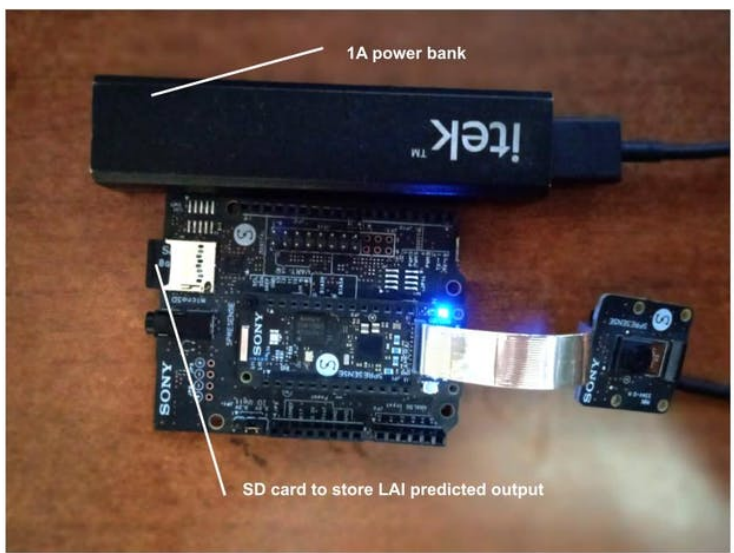

Fig. 40. Battery Operated Telemetry system using SD card to store LAI predicted data

The SD card storage on the Sony Spresense can store results of all LAI acquired from over plants in remote laboratories or semi-autonomous/autonomous hydroponic system. The built system is stationary, but a mobile solution can be designed to acquire images corresponding to GPS information tagged with the plant through Sony Spresense. This mobile autonomous system can use and store LAI information per plant collected at specific GPS co-ordinates. There are differentiably plenty 
applications in the field of auonomous monitoring and growth estimation systems fulfilling UN's SDG's.

\section{Data AVAilability:}

All Raw Image Datasets are available at Dataset Dashboard

All Code and model used for LAI and Biomass estimation is available at - github

EdgeImpulse Public Dashboard - Studio 1

Studio 2

\section{REFERENCES}

[1] R. M. Bilder, F. w. Sabb, T. D. Cannon, E. D. London, J. D. Jentsch, D. S. Parker, R. A. Poldrack, C. Evans, and N. B. Freimer, "Phenomics: The systematic study of phenotypes on a genome-wide scale," $P M C$, vol. 164, no. 1, pp. 30-42, January 2009. [Online]. Available: https://www.ncbi.nlm.nih.gov/pmc/articles/PMC2760679/

[2] A. A. Gomaa1 and Y. M. A. El-Latif, "Early prediction of plant diseases using cnn and gans," (IJACSA) International Journal of Advanced Computer Science and Applications, vol. 12, no. 5, 2021. [Online]. Available: https://thesai.org/Publications/ViewPaper?Volume= $12 \&$ Issue $=5 \&$ Code $=$ IJACSA $\&$ SerialNo $=63$

[3] I. Sa, M. Popovi, R. Khanna, Z. Chen, P. Lottes, F. Liebisch, J. Nieto, C. Stachniss, A. Walter, and R. Siegwart, "Weedmap: A large-scale semantic weed mapping framework using aerial multispectral imaging and deep neural network for precision farming," Remote Sensing MDPI, no. 1, pp. 30-42, September 2018. [Online]. Available: https://www.mdpi.com/2072-4292/10/9/1423

[4] Z. Lingxian, X. Zanyu, X. Dan, M. Juncheng, C. Yingyi, and F. Zetian, "Phenomics: The systematic study of phenotypes on a genome-wide scale," Horticulture Research, vol. 7, no. 124, August 2020. [Online]. Available: https://www.nature.com/articles/s41438-020-00345-6\#citeas

[5] L. Drees, L. V. Junker-Frohn, J. Kierdorf, and R. Roscher, "Temporal prediction and evaluation of brassica growth in the field using conditional generative adversarial networks," arXiv, May 2021. [Online]. Available: https://arxiv.org/abs/2105.07789

[6] R. Yasrab, J. Zhang, P. Smyth, and M. P. Pound, "Predicting plant growth from time-series data using deep learning," Remote Sensing, vol. 13, no. 3, p. 331, Jan 2021. [Online]. Available: http://dx.doi.org/10.3390/rs13030331

[7] R. MM, C. D, G. Z, K. C, and C. M, "Advanced phenotyping and phenotype data analysis for the study of plant growth and development." Front. Plant Sci., 2015. [Online]. Available: https://www.frontiersin.org/articles/10.3389/fpls.2015.00619/full\#B118

[8] M. Fawakherji, C. Potena, I. Prevedello, A. Pretto, D. D. Bloisi, and D. Nardi, "Data augmentation using gans for crop/weed segmentation in precision farming," in 2020 IEEE Conference on Control Technology and Applications (CCTA), 2020, pp. 279-284.

[9] M. Teobaldelli, Y. Rouphael, G. Fascella, V. Cristofori, C. M Rivera, and B. Basile, "Developing an accurate and fast nondestructive single leaf area model for loquat (eriobotrya japonica lindl) cultivars," Plants, vol. 8, no. 7, 2019. [Online]. Available: https://www.mdpi.com/2223-7747/8/7/230

[10] A. Bauer, A. G. Bostrom, J. Ball, C. Applegate, T. Cheng, S. Laycock, S. M. Rojas, J. Kirwan, and J. Zhou, "Combining computer vision and deep learning to enable ultra-scale aerial phenotyping and precision agriculture: A case study of lettuce production," Horticulture Research, vol. 6, no. 1, p. 70, Jun 2019. [Online]. Available: https://doi.org/10.1038/s41438-019-0151-5

[11] M. P. Pound, J. A. Atkinson, A. J. Townsend, M. H. Wilson, M. Griffiths, A. S. Jackson, A. Bulat, G. Tzimiropoulos, D. M. Wells, E. H. Murchie, T. P. Pridmore, and A. P. French, "Deep machine learning provides stateof-the-art performance in image-based plant phenotyping," GigaScience, vol. 6, no. 10, pp. 1-10, Oct 2017, 29020747[pmid]. [Online]. Available: https://pubmed.ncbi.nlm.nih.gov/29020747 


\section{Figures}

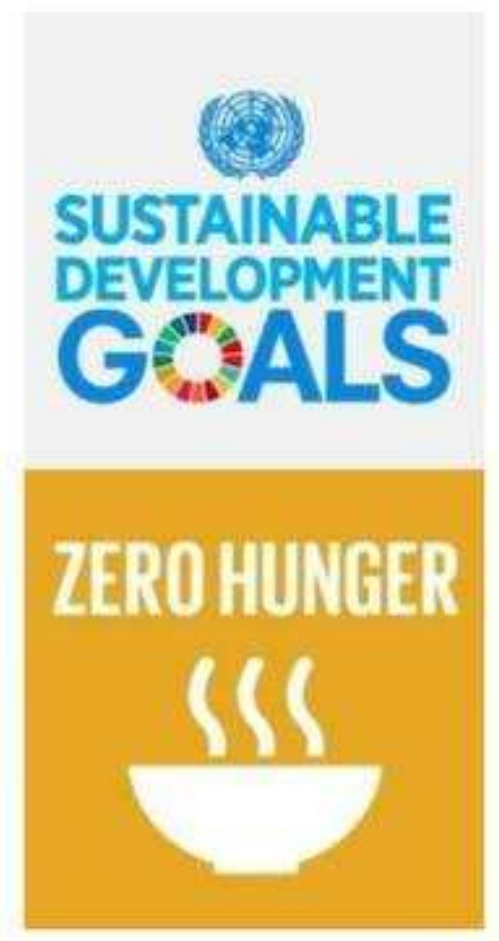

- By 2030 , double the agricultural productivity and incomes of small-scale food producers, in particular women, indigenous peoples, family farmers, pastoralists and fishers, including through secure and equal access to land, other productive resources and inputs, knowledge, financial services, markets and opportunities for value addition and non-farm employment

- By 2030, ensure sustainable food production systems and implement resilient agricultural practices that increase productivity and production, that help maintain ecosystems, that strengthen capacity for adaptation to climate change, extreme weather, drought, flooding and other disasters and that progressively improve land and soll quality

\section{SONY III E EDGE IMPULSE}

\section{Figure 1}

Explaining UN's SDG's and how this project is bringing it one step closer to life
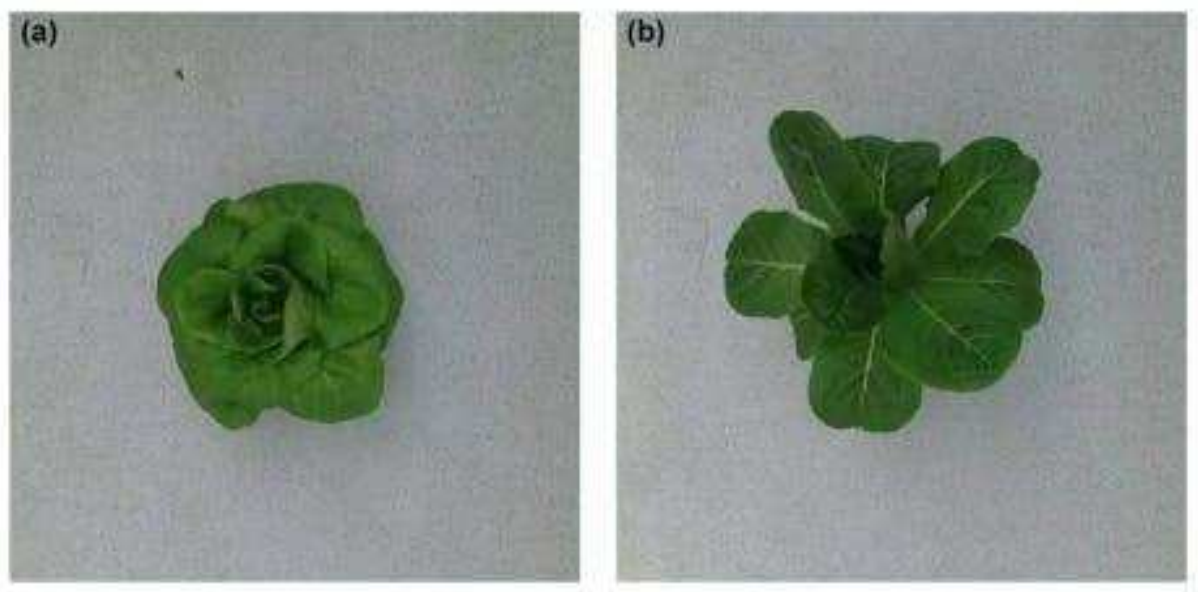

\section{Figure 2}

(Left) Lettuce Flandria Cultivar. (Right) Lettuce Tiberius Cultivar. source - [Growth monitoring of greenhouse lettuce paper] 


\section{2times}
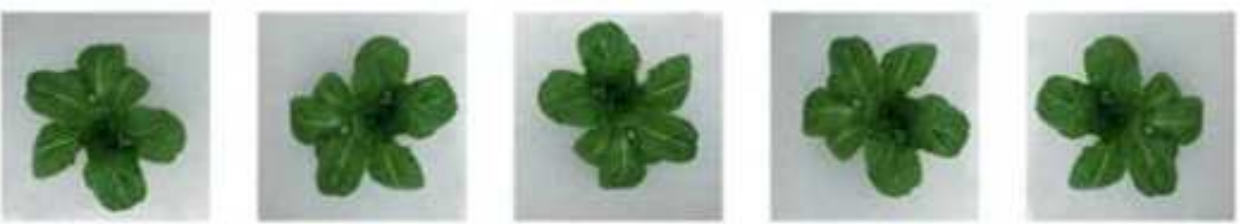

\section{1 times}
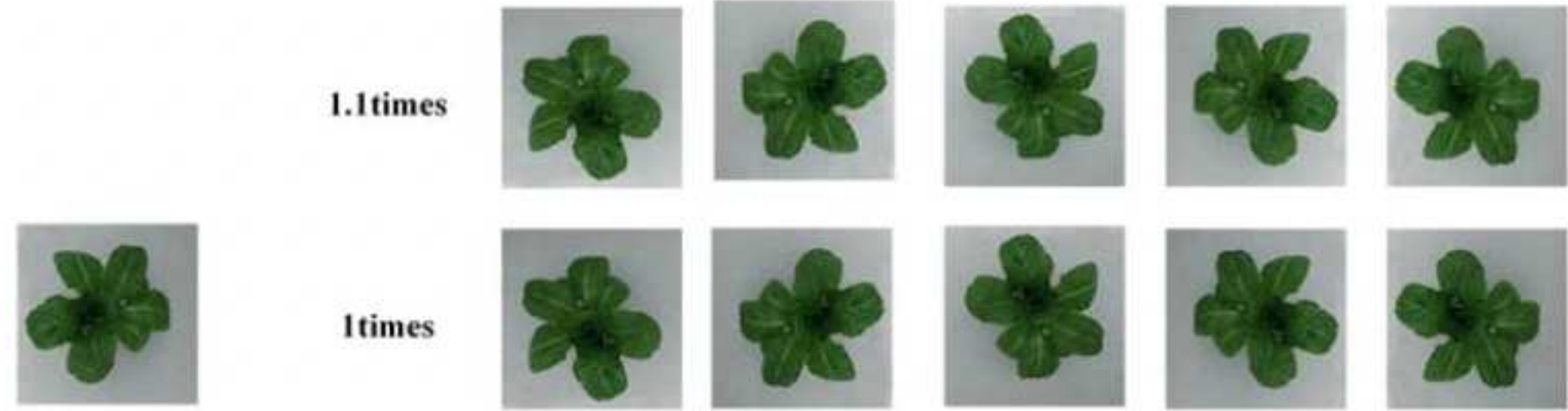

Original image

Itimes
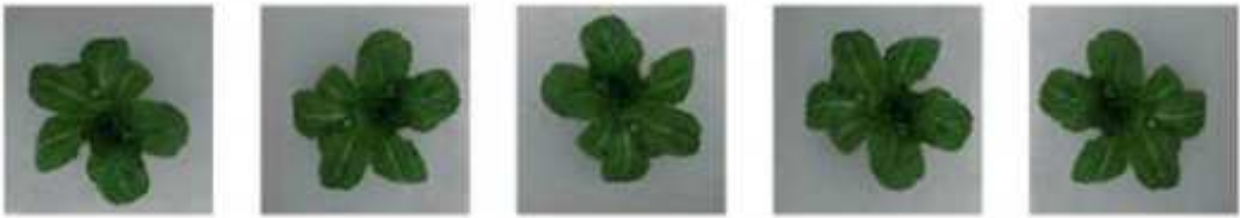

\section{$\uparrow \begin{gathered}0.8 \text { times } \\ \text { brightness } \\ \text { enhancement }\end{gathered}$}
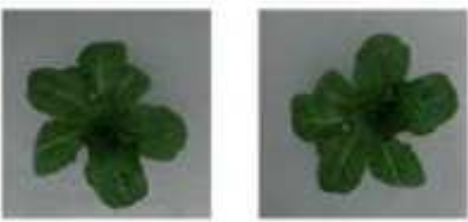

Rotate $180^{\circ}$
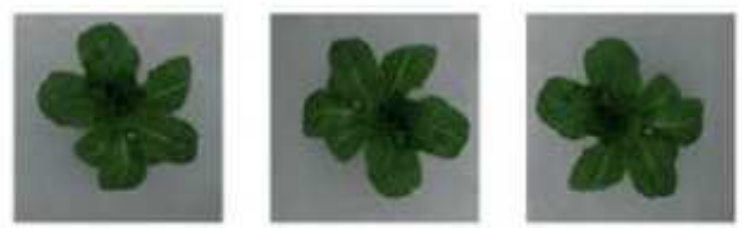

Augment

Rotate $90^{\circ}$

Rotate $270^{\circ}$

Flip horizontal Flip vertical

Figure 3

source - [Growth Monitoring of Greenhouse Lettuce] Lingxian Zhang et al. 


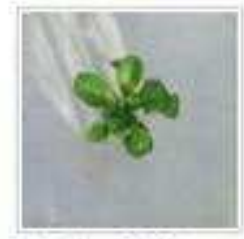

Color:6-29.jpg

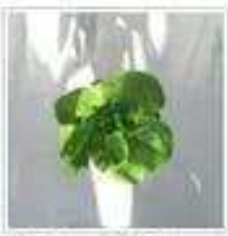

Color-6-35.jpg

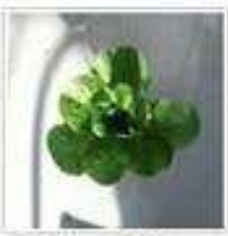

Color-6-41.jpg

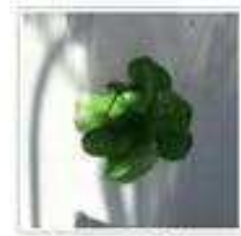

Color-6-47.jpg

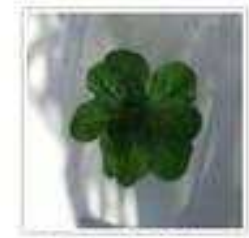

Color-6-53.jpg

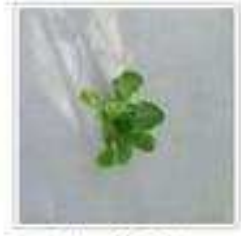

Color-6-30.jpg
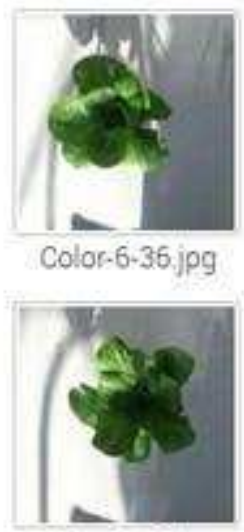

Color-6-42.jpg
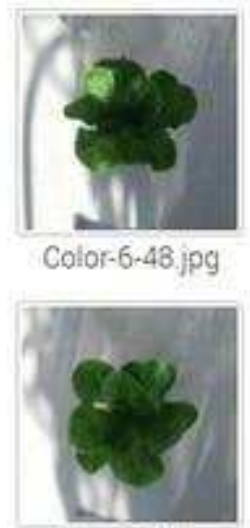

Color-6-54.jpg

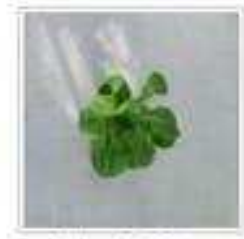

Color-6-31,jpg

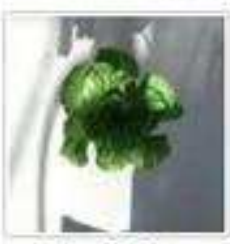

Color-6-37.jpg

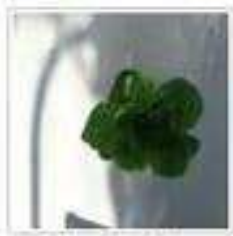

Color-6-43.jpg
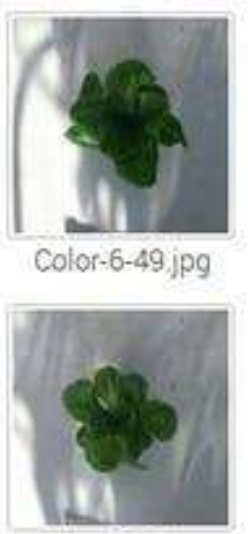

Color-6-55.jpg

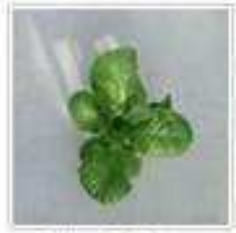

Color-6-32.jpg

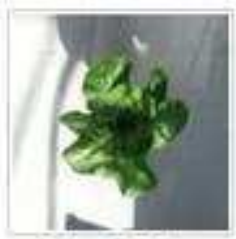

Color-6 38 jpg

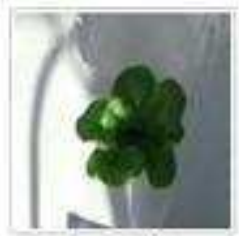

Color-6-44.jpg

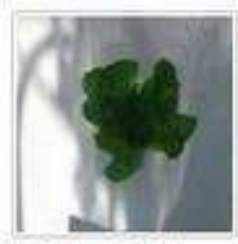

Color-6-50.jpg

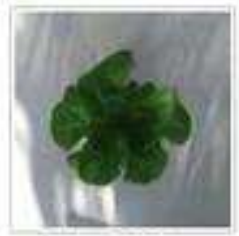

Color-6-56.jpg

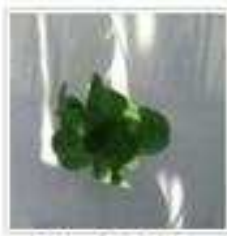

Color-6-33.jpg

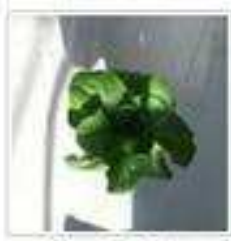

Color-6-39.jpg

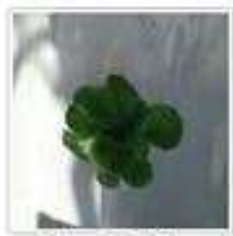

Color-6-45.jpg

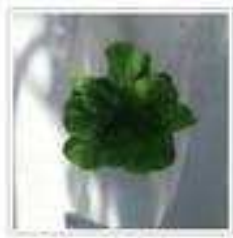

Color-6-51.jpg

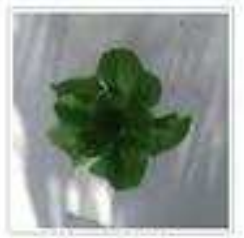

Color-6-57.jpg

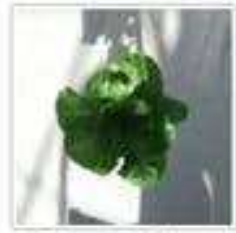

Color-6-34.jpg

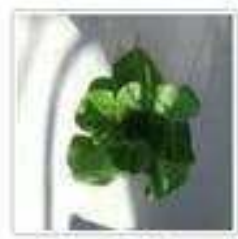

Color-6-40.jpg

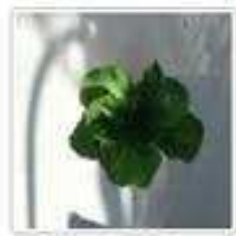

Color-6-46.jpg

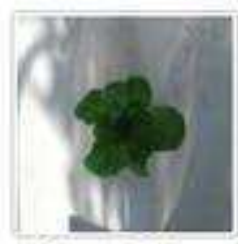

Color-6-52.jpg

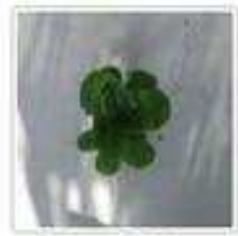

Color-6-58.jpg

\section{Figure 4}

Illustrates how raw images show varying illuminance which might be a major drawback for the neural network. 


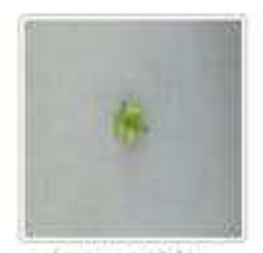

lettuce13.jpg

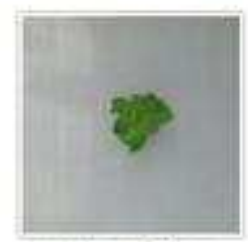

lettuce 19.jpg

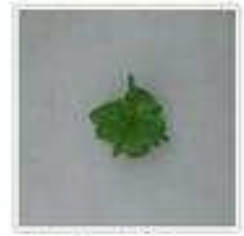

lettuce 25.jpg

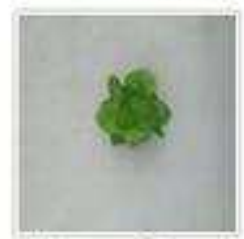

lettuce31.jpg

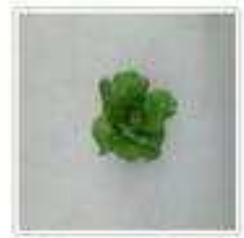

lettuce $37 . j \mathrm{pg}$

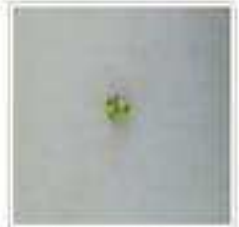

lettuce 14.jpg

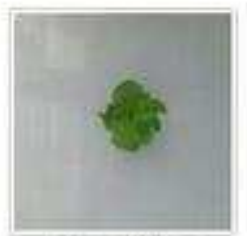

lettuce20.jpg

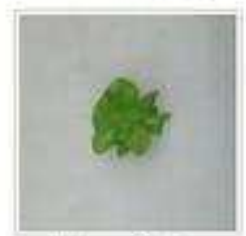

lettuce26.jpg

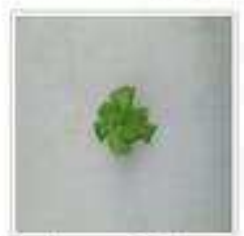

lettuce $32 . j p g$

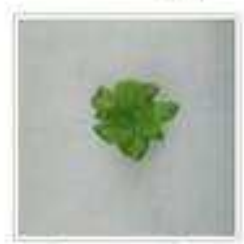

lettuce $38 . j p g$

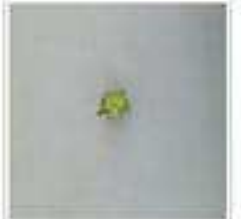

lettuce15.jpg

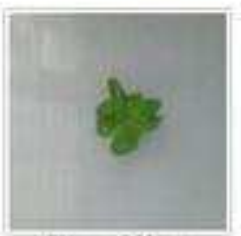

lettuce21.jpg

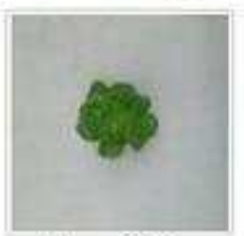

lettuce27.jpg

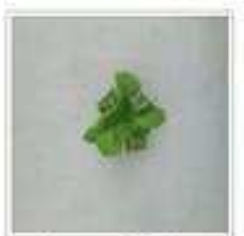

lettuce33.jpg

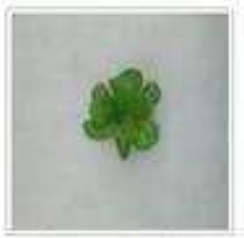

iettuce39.jpg

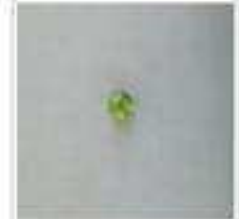

lettuce16.jpg

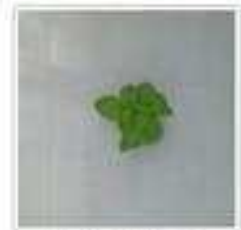

lettuce22.jpg

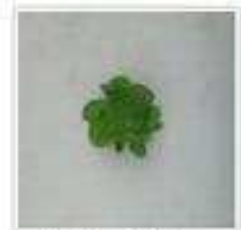

lertuce28.jpg

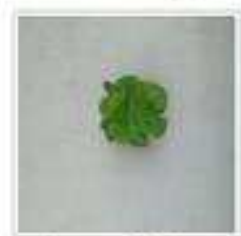

lettuce 34.jpg

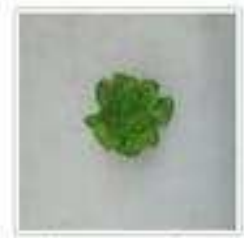

lettuce40,jpg

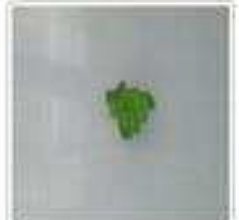

lettuce17.jpg

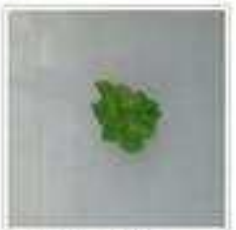

lettuce23.jpg

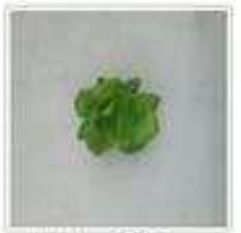

lettuce29.jpg

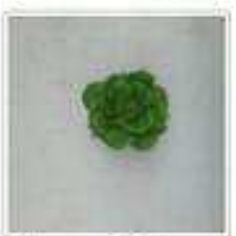

lettuce35.jpg

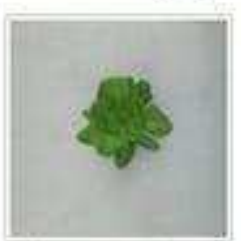

lettuce41.jpg

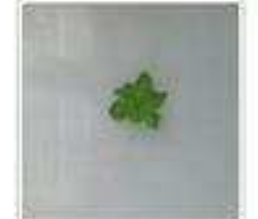

lettuce18.jpg

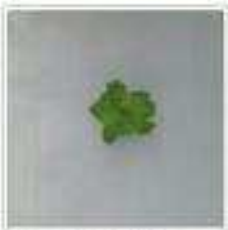

lettuce24.jpg

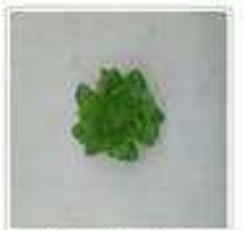

lettuce30.jpg

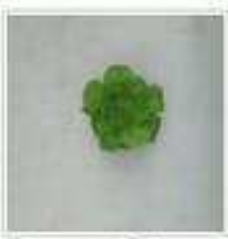

lettuce 36.jpg

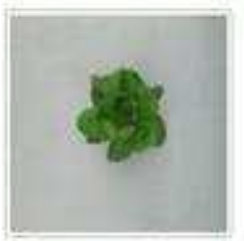

lettuce42.jpg

Figure 5

Synthesized Images for Neural Network possessing equalized illuminance, RGB Channels and saturation to maintain consistent Data input for Neural Network. 


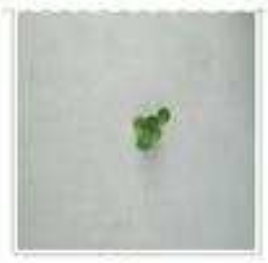

7.jpg

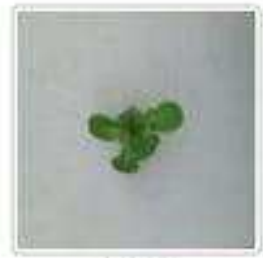

13.jpg

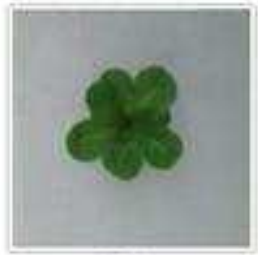

19.jpg

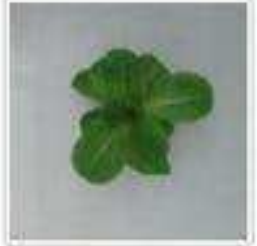

25.jpg

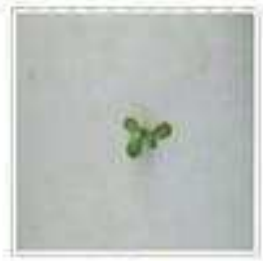

8.jpg

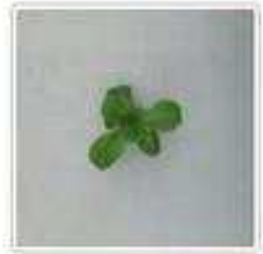

14.jpg

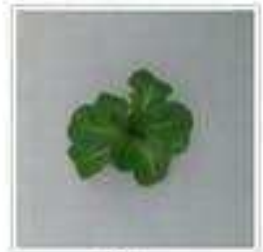

20.jpg

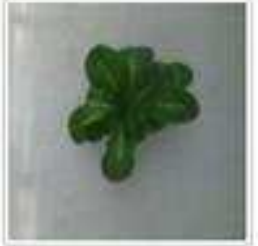

26.jpg

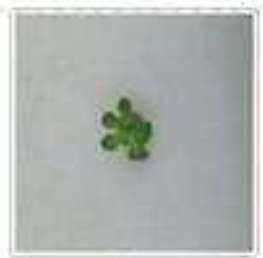

9.jpg

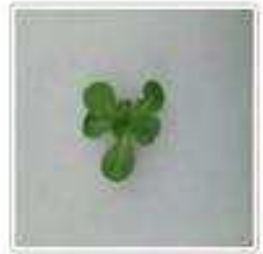

15.jpg

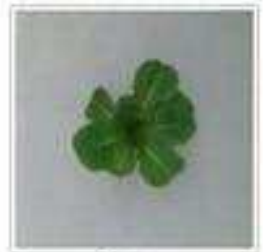

21.jpg

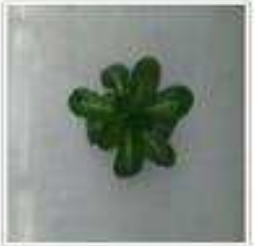

27.jpg

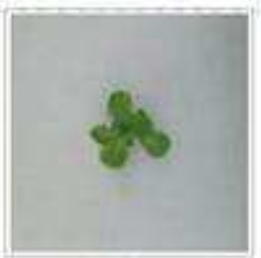

10.jpg

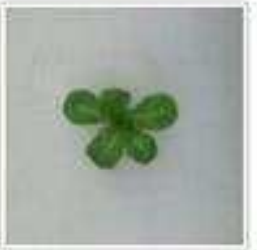

16.jpg

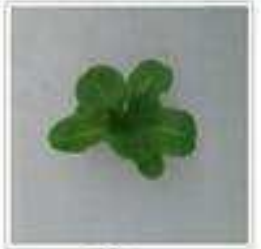

22.jpg

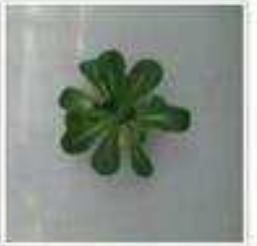

28.jpg

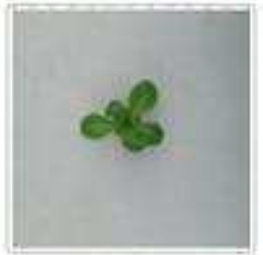

11.jpg

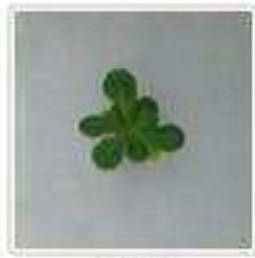

17.jpg

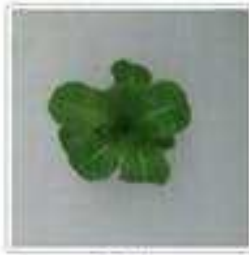

23.jpg

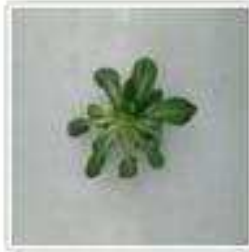

29.jpg

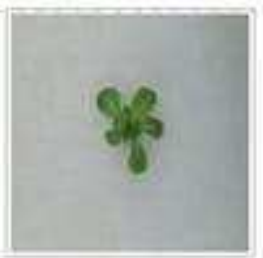

$12 . j p g$

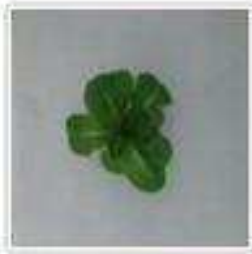

18.jpg

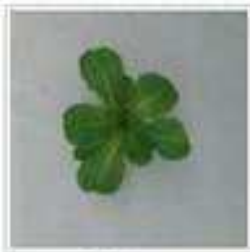

24.jpg

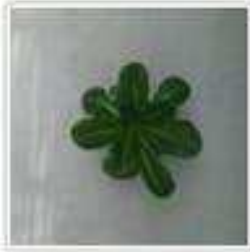

30.jpg

\section{Figure 6}

Lettuce Tiberius dataset used for training model specific to test on Tiberius plant subtype. 


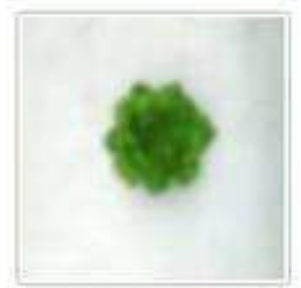

50.jpg

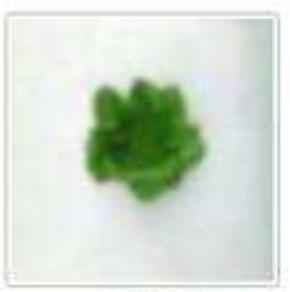

56.jpg

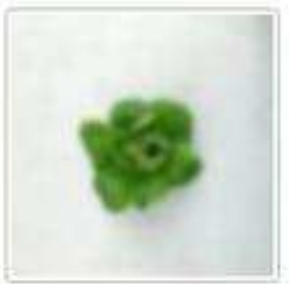

62.jpg

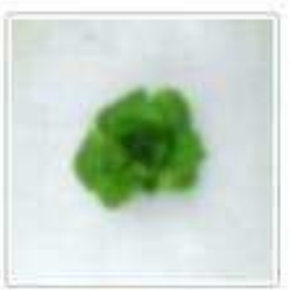

68.jpg

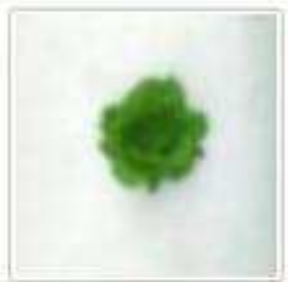

51.jpg

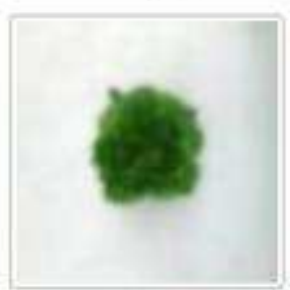

57.jpg

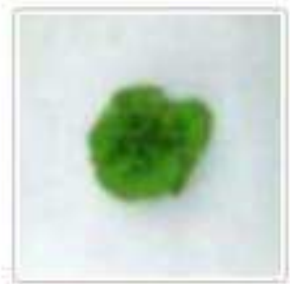

63.jpg

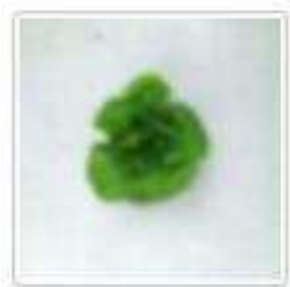

69.jpg

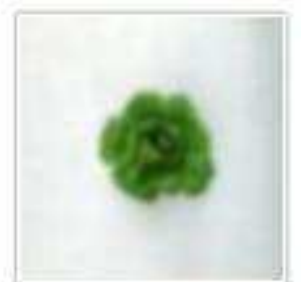

52.jpg

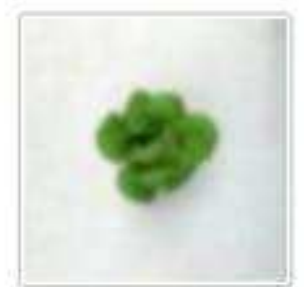

58.jpg

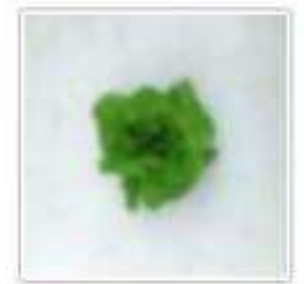

64.jpg

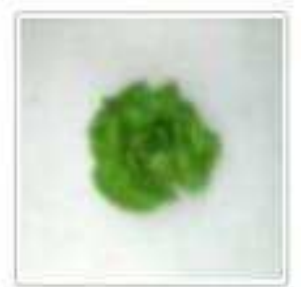

70.jpg

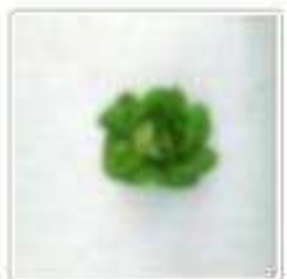

53.jpg

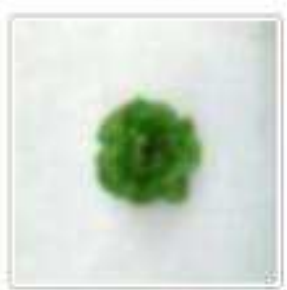

59.jpg

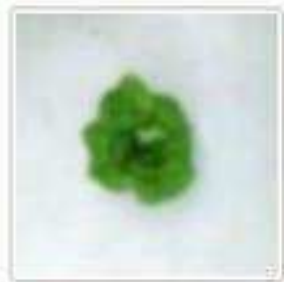

65.jpg

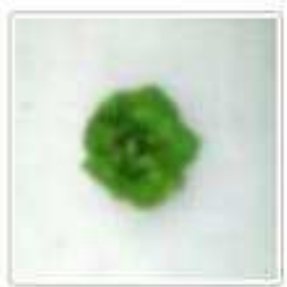

71.jpg

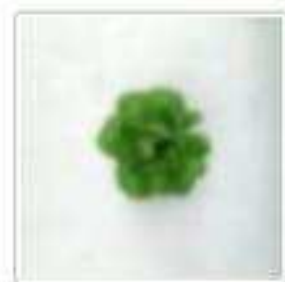

54.jpg

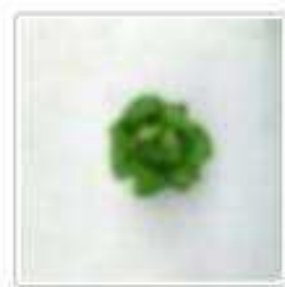

$60 . j p g$

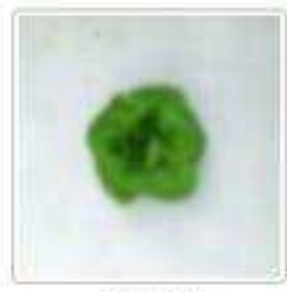

66.jpg

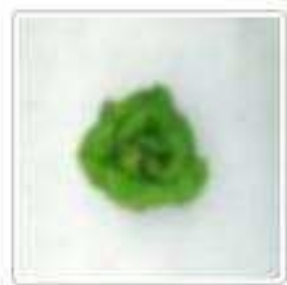

72.jpg

Figure 7

A translational motion blur of 0.01 added to synthesized dataset. 


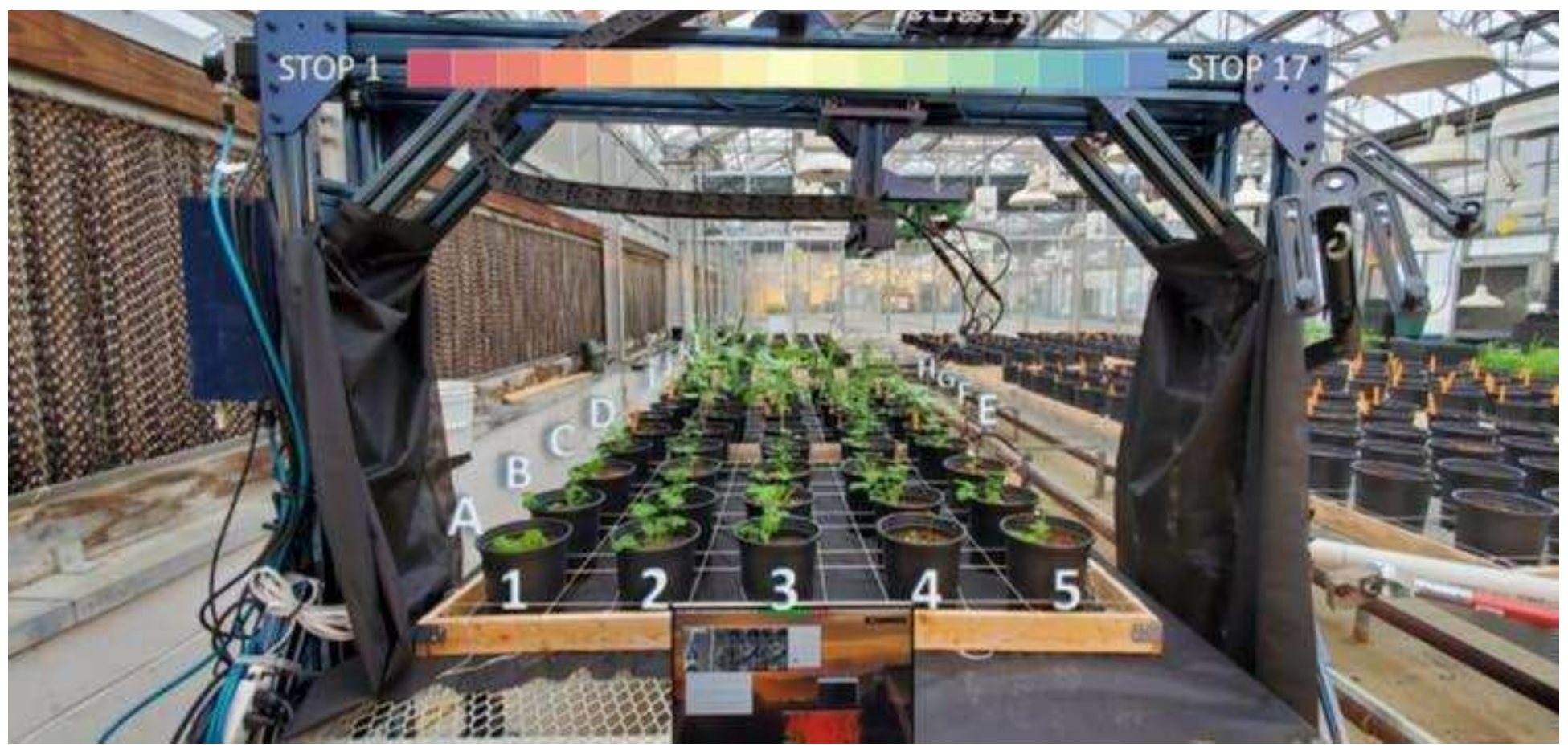

Figure 8

Translational Data Collection from motorized plates. Source - Paula Ramos Et Al. Precision Sustainable Agriculture

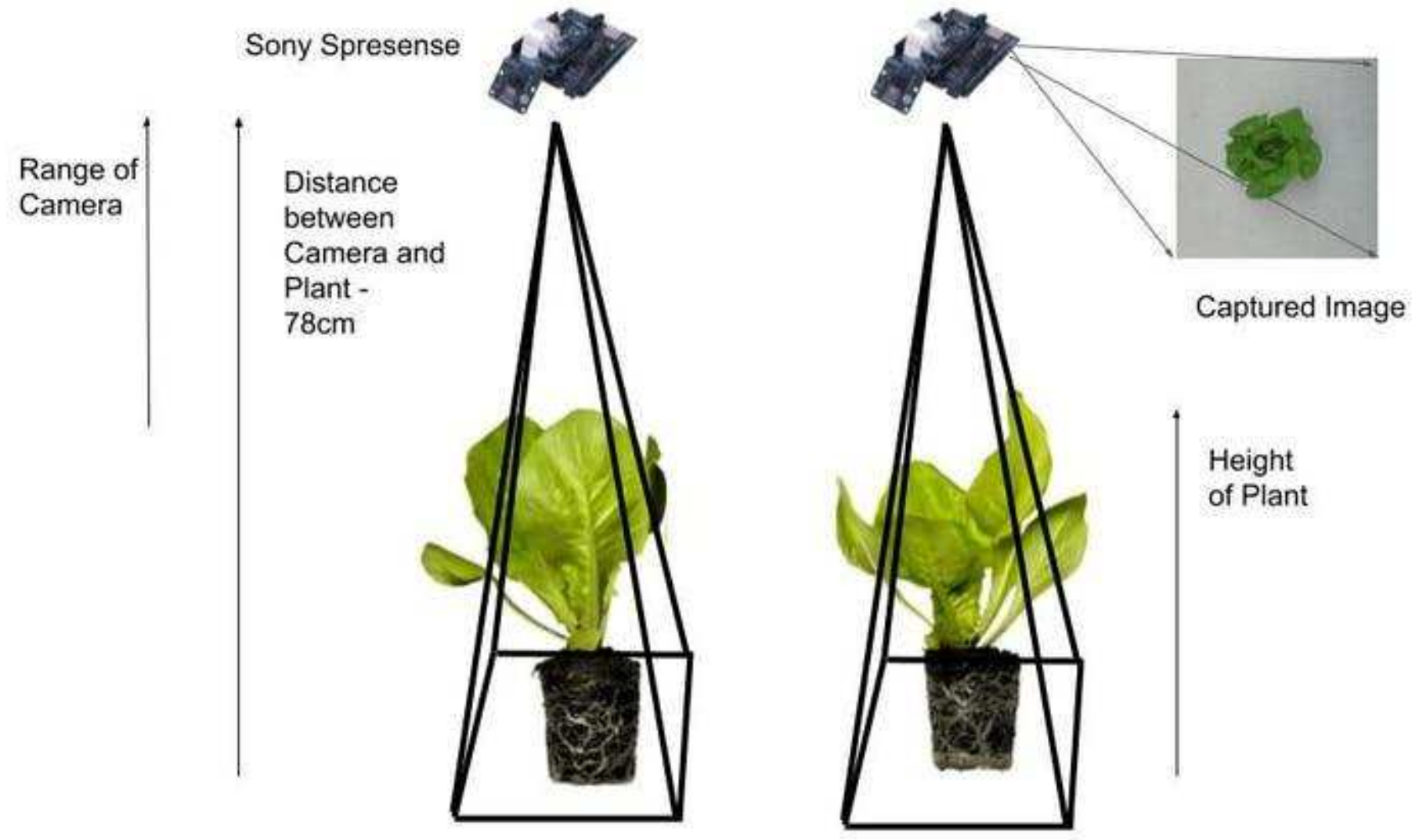

Figure 9 
Field of Object in Image frame and image capturing process illustrated

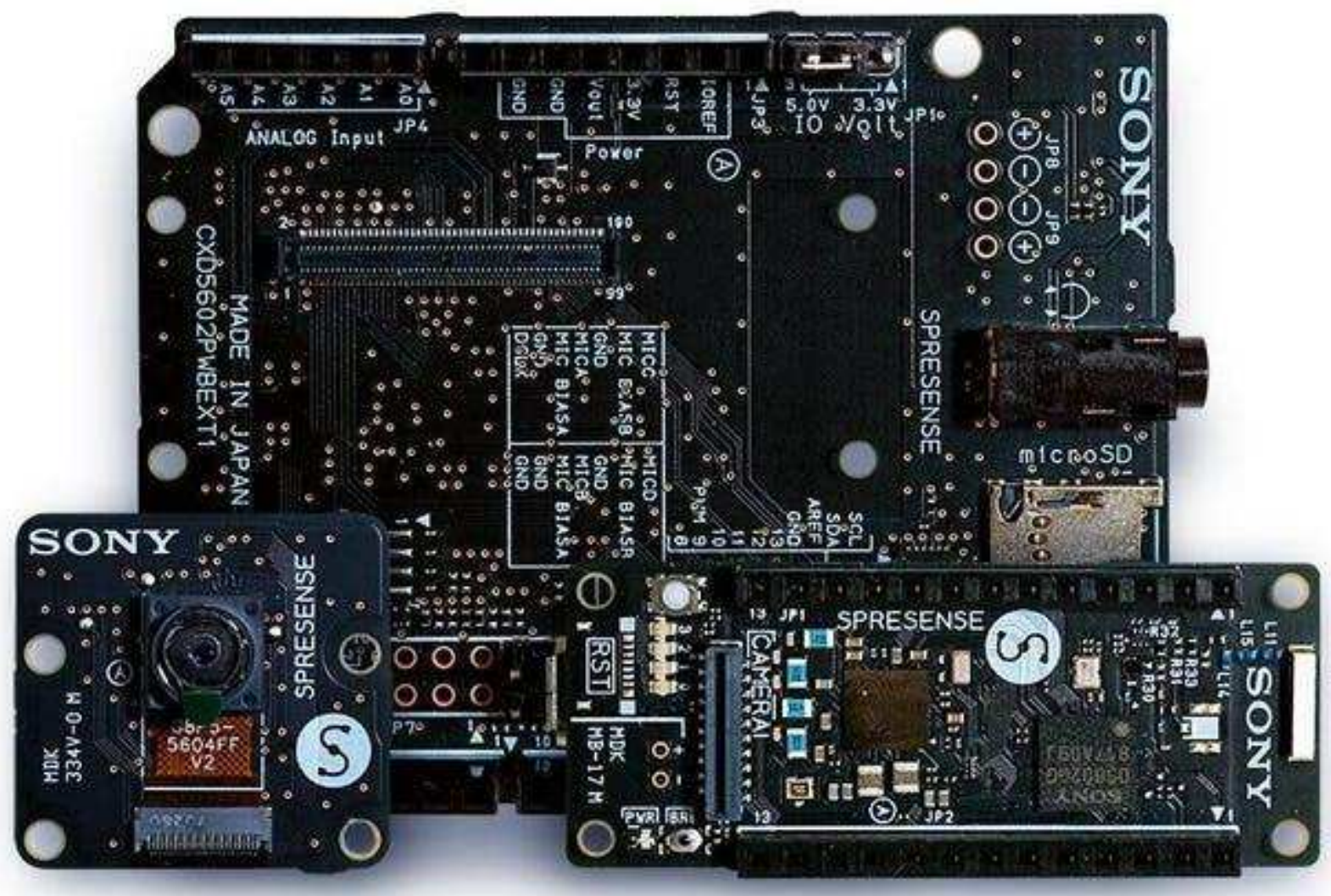

Figure 10

The Sony Spresense suite featuring a main board, camera shield and neural processing board 
Sony Spresense Camera setup for collection and test on on Deployed Regression Model
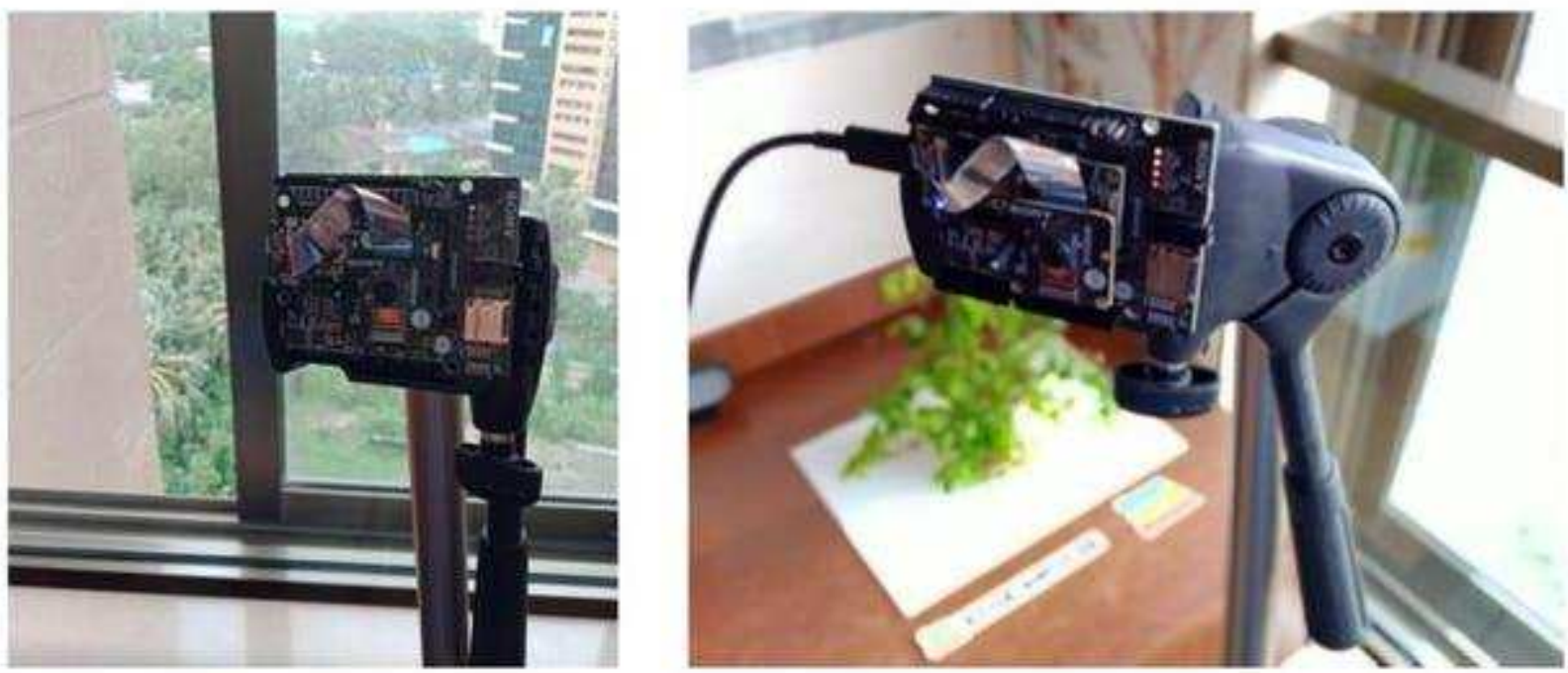

\section{Figure 11}

Live Classification and data accumulation process for the regression model

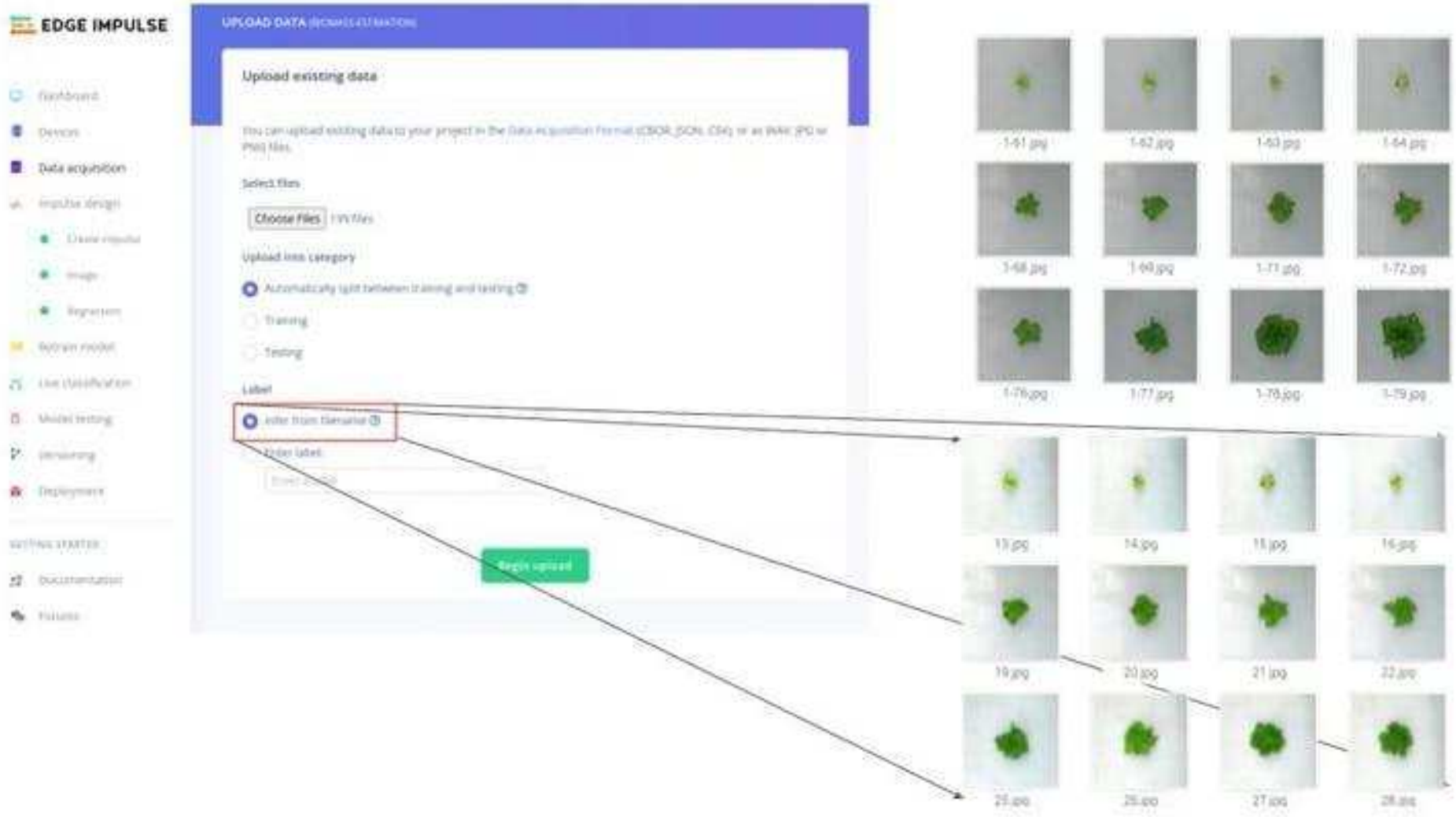


Edgelmpulse Dashboard Data ingestion process

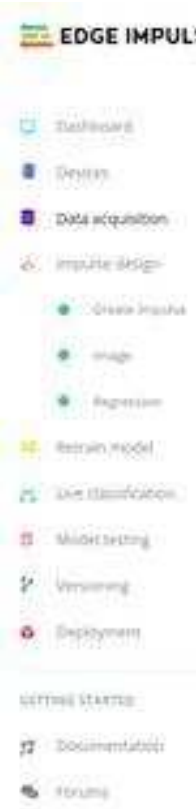

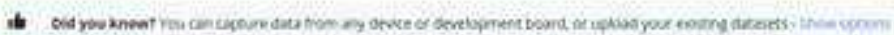

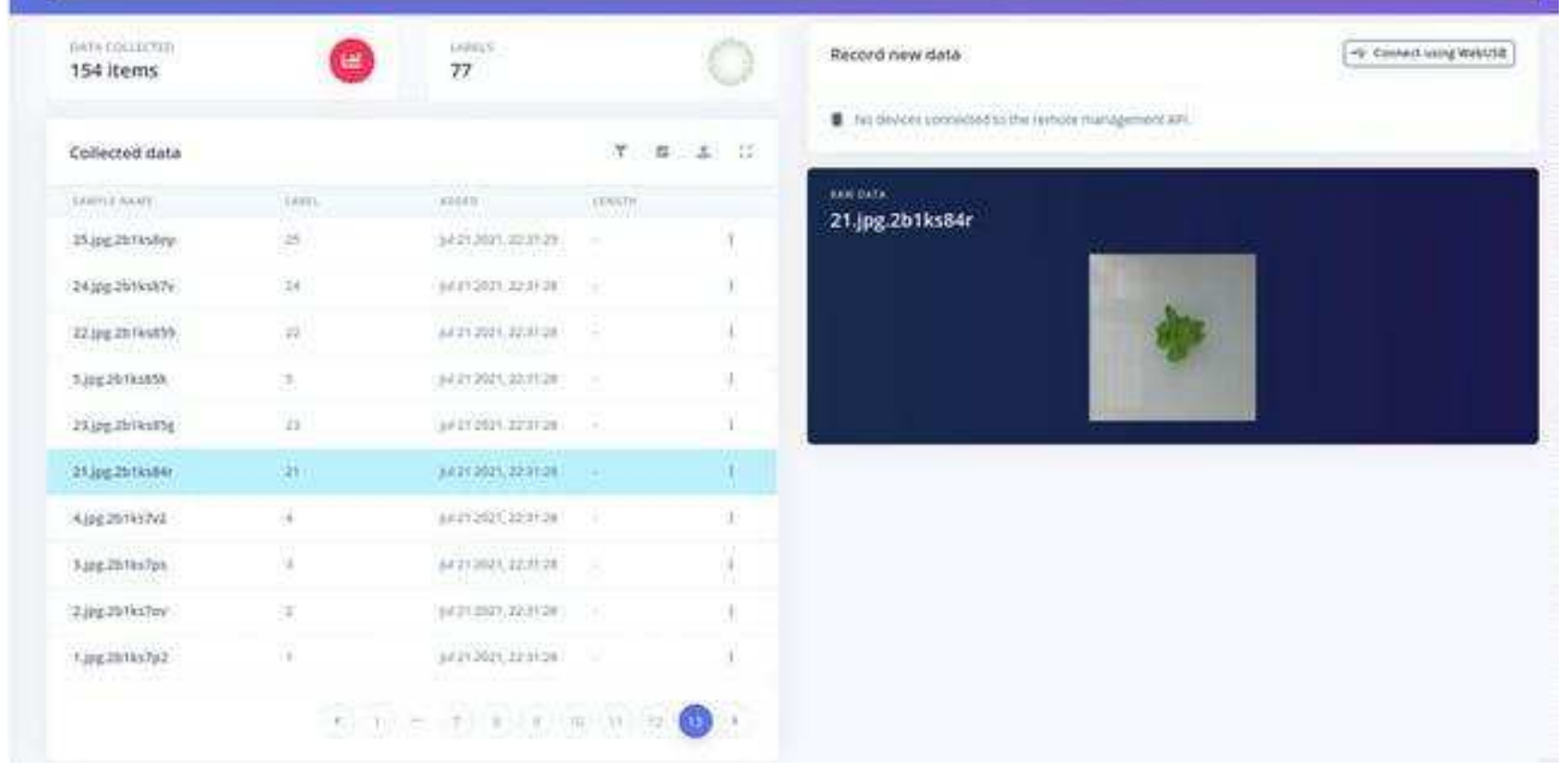

Figure 13

Edgelmpulse Dashboard after the dataset is ingested

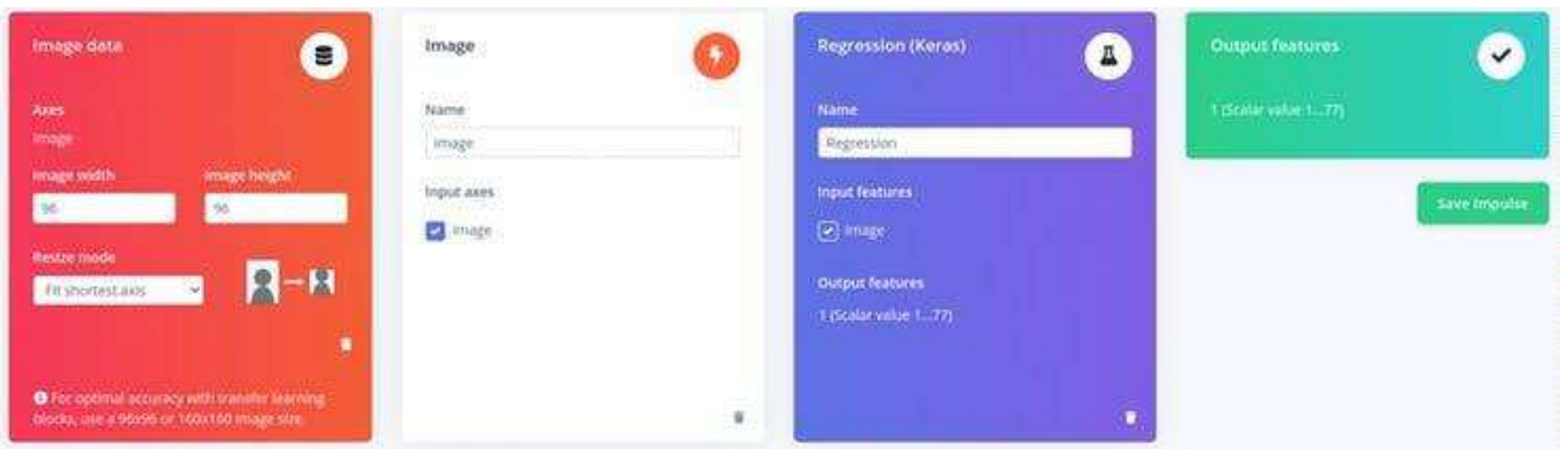

Figure 14

Creating an Impulse, designing CNN pipeline. 


\section{Raw features}

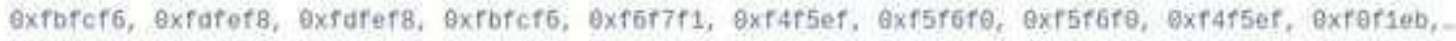

\section{DSP result}

image

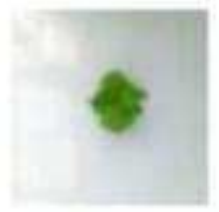

Processed features

$0.9843,8.9882,8.9647,0.9922,8.9951,8.9725,0.9922,8.9961,0.9725,8.9843,8.9882,0.9647,0.9$.

\section{Figure 15}

Processing Raw features as processed features

Feature explorer (75 samples)

Xaks

Woustustiea ower I

$:$ is

.

: 4

- 5

- 60

-01
$-\quad 61$

- 65

- 65

- is

- is

$: \quad 67$

- es

, 7

$: \geqslant$

12

- in

- 75

32. ipe 274h\%m

tabel 12

Verssaiple

iavifoututs:

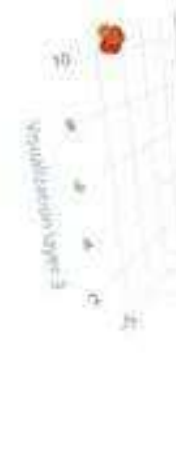

(1)

ZAxos

\section{Features Extracted for Lettuce} Tiberius Dataset
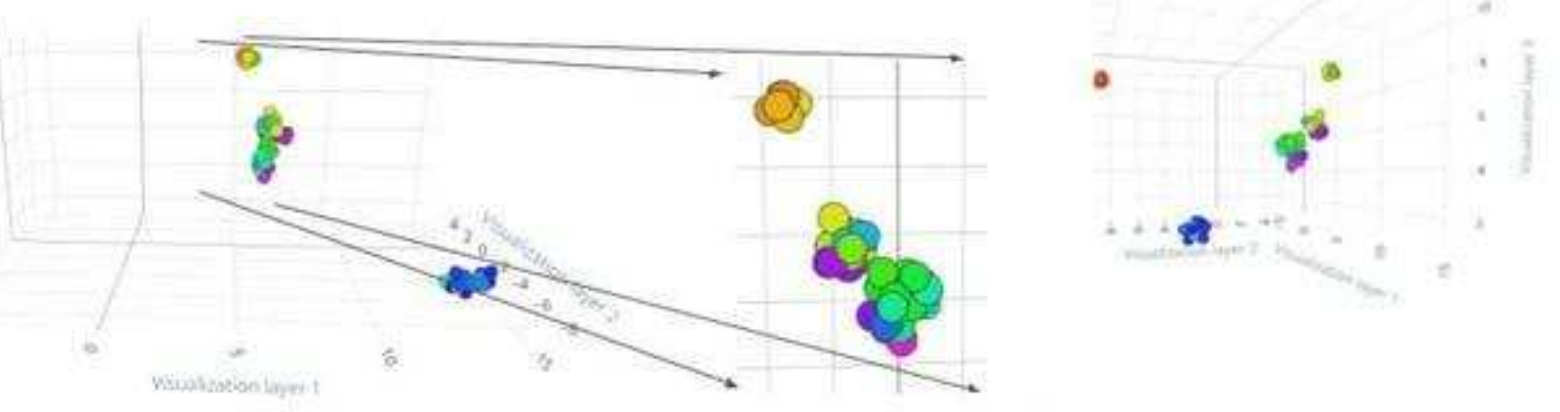

Figure 16
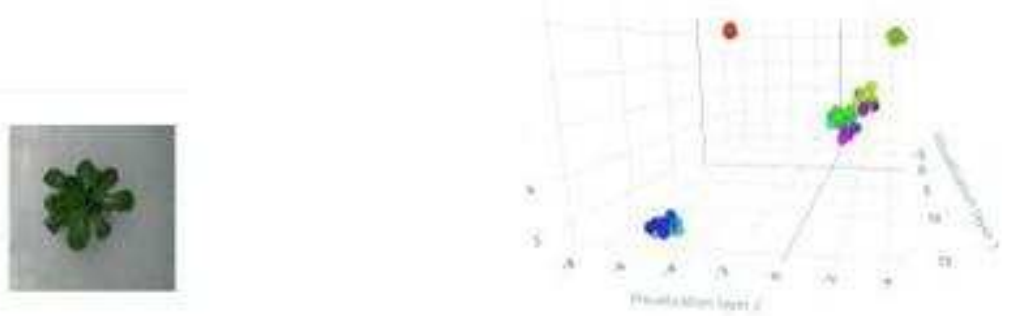
vesateratien (rerr

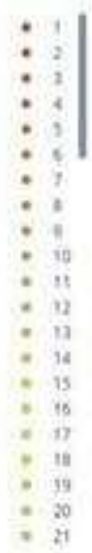

15 jpg $2 \mathrm{~b} 1 \mathrm{ksavd}$

Labecis

Verwinates

himyeoture: yavis

2. Axis
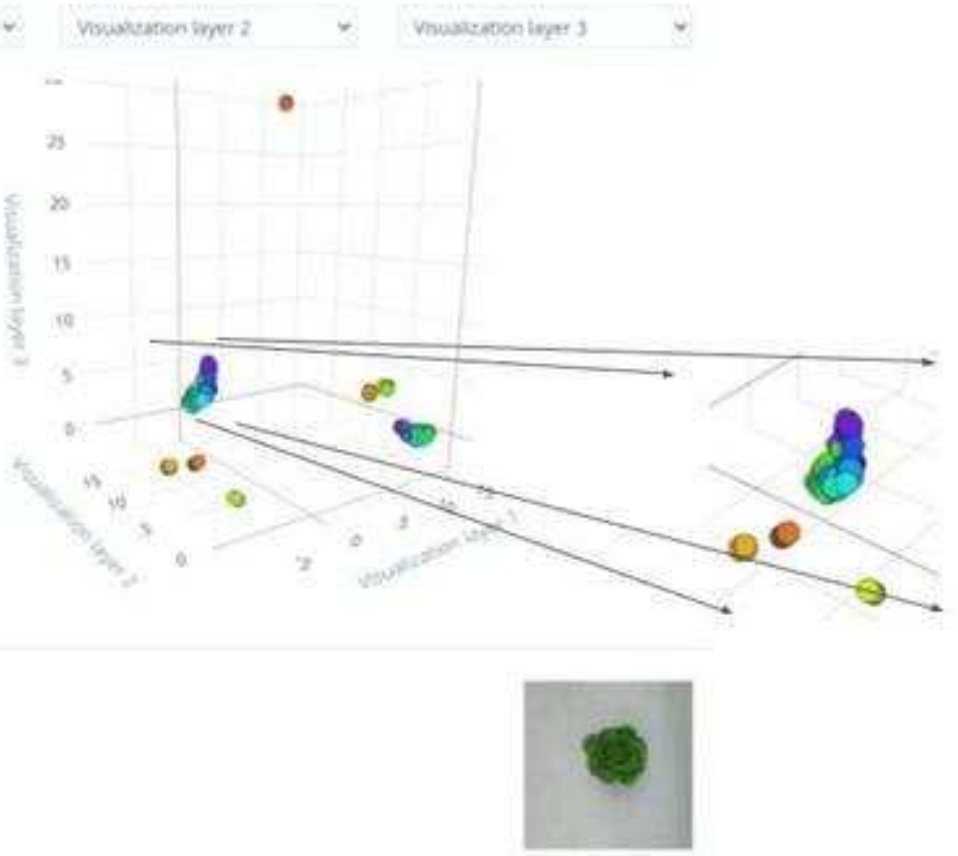

\section{Features Extracted for Lettuce} Flandria Dataset
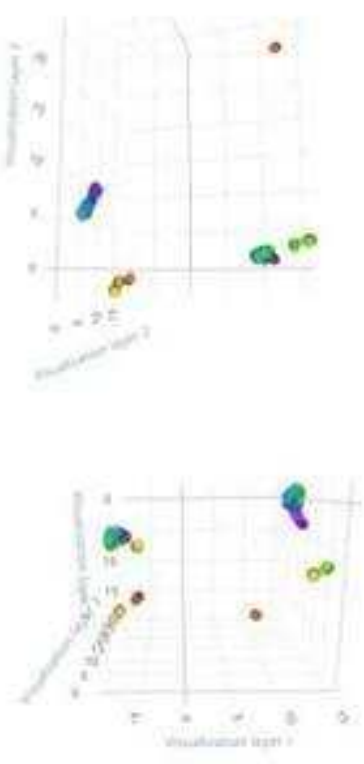

Figure 17

Pre-processing and Feature Extraction of Lettuce Flandria Dataset.

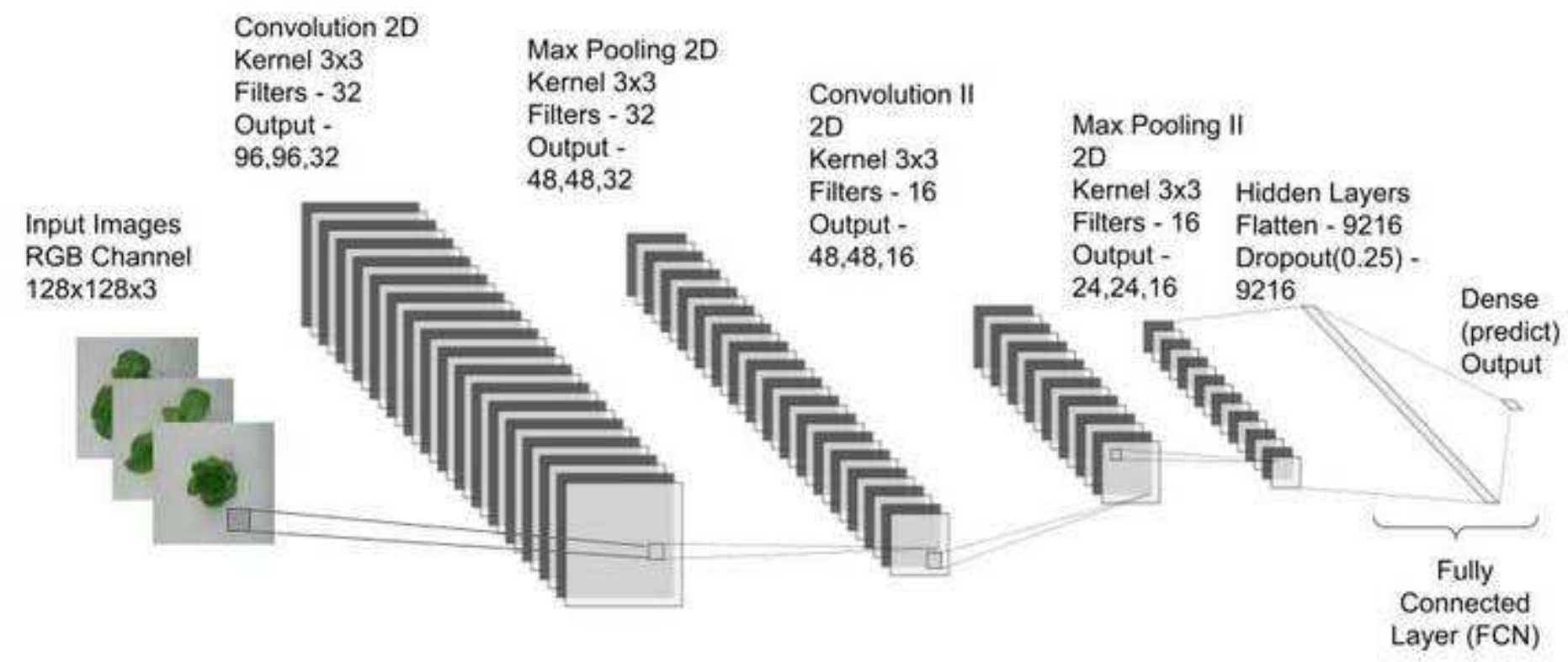


Figure 18

Representation of the CNN Architecture used for Training the Regression Model

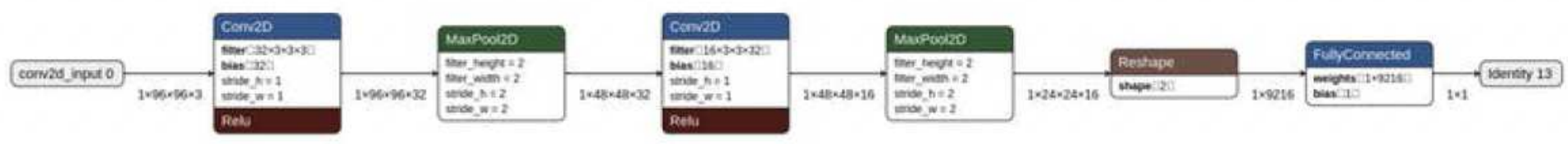

Figure 19

Netron Neural Network Render of the CNN Architecture

Neural network architecture

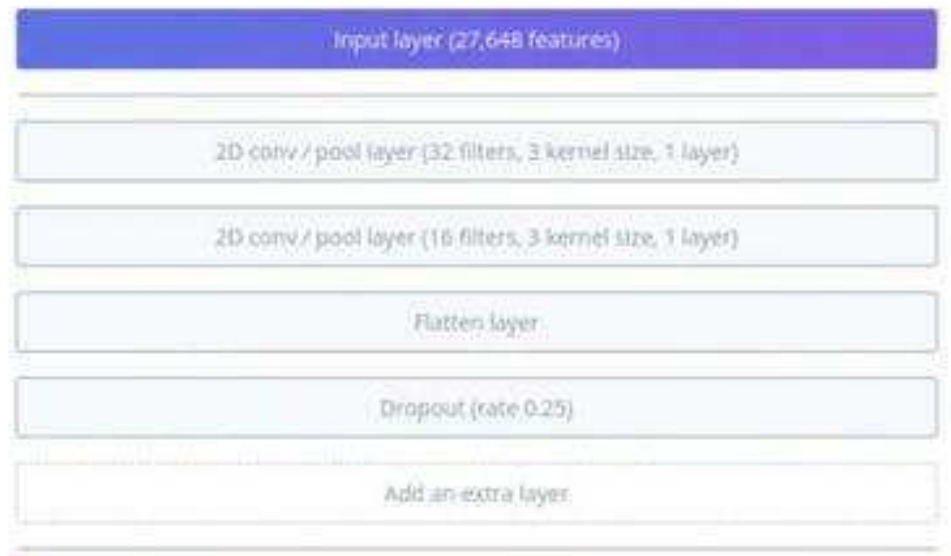

outputajer (1) fevtures)

Geret troining

\begin{tabular}{|c|c|c|}
\hline \multicolumn{3}{|c|}{ 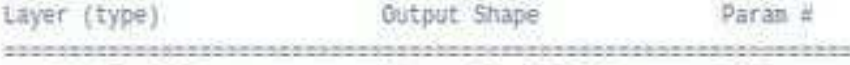 } \\
\hline entrit $(\cos 20)$ & (None, $96,96,32$ ). & 896 \\
\hline $\max$ pooting2d (Naxpooiling20) & (Wone, 48, 48, 32) & $\theta$ \\
\hline corw2t:1 (Conv20) & (None, $48,48,16$ ) & 4624 \\
\hline aax_pooting2d_1 (NaxPooting? & (None, $24,24,16$ ) & 当 \\
\hline flatten (Flatten) & (Wone, 9215) & g \\
\hline dropout (Dropout) & (None, 9216) & $\theta$ \\
\hline y_pred (Dense) & (None, 1) & 9217 \\
\hline \multicolumn{3}{|l|}{ Totat parans: 14,737} \\
\hline \multicolumn{3}{|l|}{ Trasnabte parans: 14,737} \\
\hline Non-trainable paraus: $B$ & & \\
\hline
\end{tabular}

Finished training.

\begin{tabular}{|c|c|c|c|c|}
\hline 4 & 20 conv / pool layer po filters, 3 kernel size, 1 ial & $\mathbb{Z}$ & $\approx \equiv$ - & - To edit no of layers, filters, \\
\hline
\end{tabular}

Figure 20

Flexible Functionality to alter the architecture of the CNN model in El Studio. 
Leaf Area Index calculations in $\mathrm{cm}^{\wedge} 2$ used as labels

Model

On-device performance (2)

Quantised INT8:

Last training performance fvalidation set

INFERENCING TIME $1,544 \mathrm{~ms}$.

PEAK RAM USAGE

$362.5 \mathrm{~K}$

FLASTIUSRGE

$38.2 \mathrm{~K}$

On-device performance (2)

Unoptimised Float32:

(3)

INFERENCING TMAF $7,268 \mathrm{~ms}$.

PLAX RUAM USABS

Growth stage day-wise from 1 to 77 Integral values used as labels

Model

On-device performance (8)

Quantised INT8:

Last training performance (valdation set:

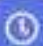

(9)

On-device performance (?)

14.71
UTRENCING TMAE $2,656 \mathrm{~ms}$.
INFERENCING TIME

$12,501 \mathrm{~ms}$.

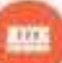

USACT $362.5 K$

\section{Unoptimised Float32:}

PEAK RAM USAGE

$1.4 \mathrm{M}$
FLASH USAGE

$80.1 \mathrm{~K}$

\section{Figure 21}

Comparing Change in model performance with labels set as biomass values, in comparison with (down) Day wise labels of growth stage of plant 


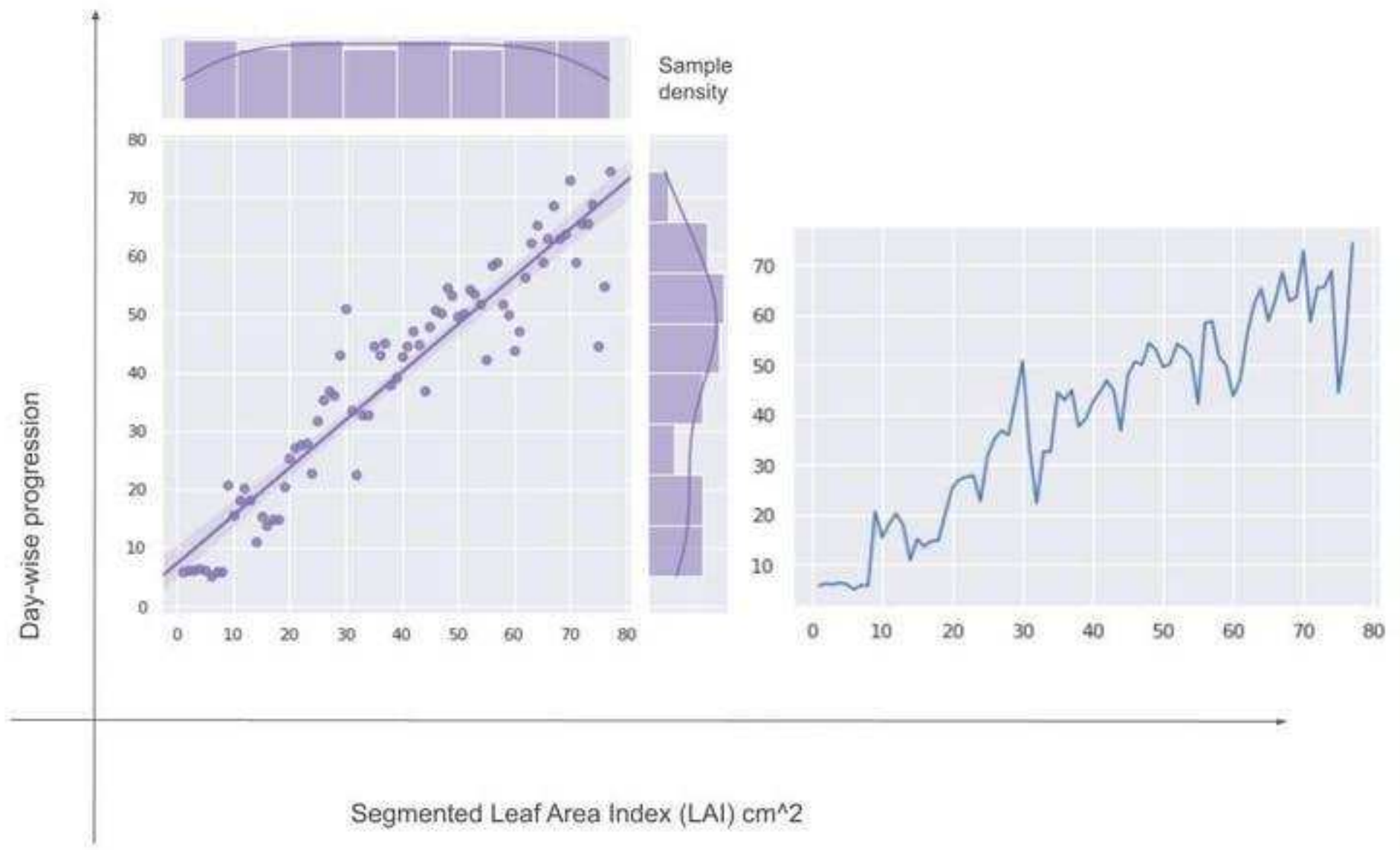

Figure 22

Relplot using Seaborn to analyze inter-relation between LAI labels and Day-wise progression labels 

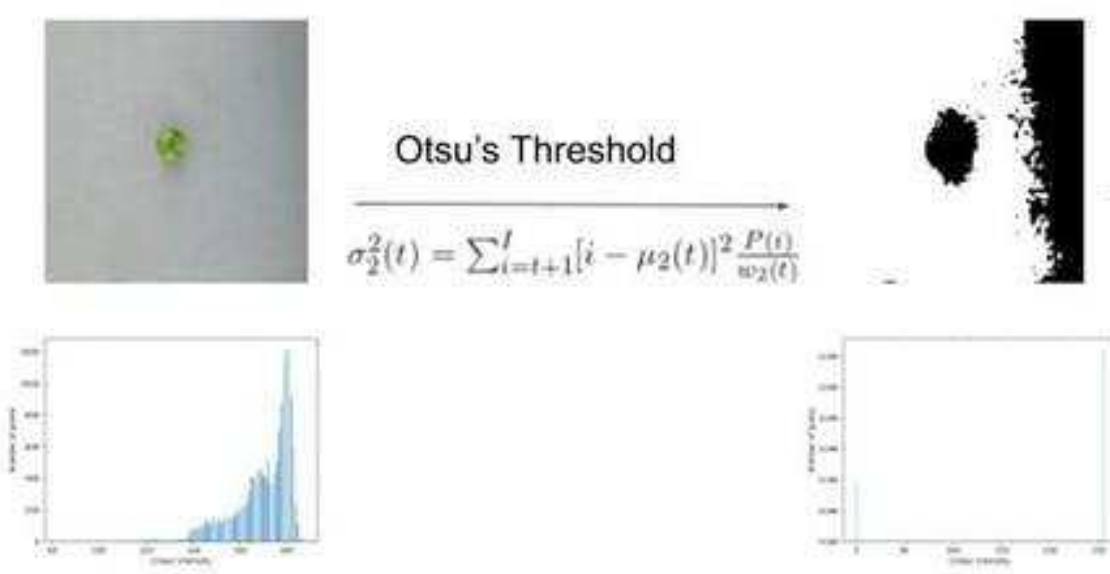

Raw Image and RGB histogram
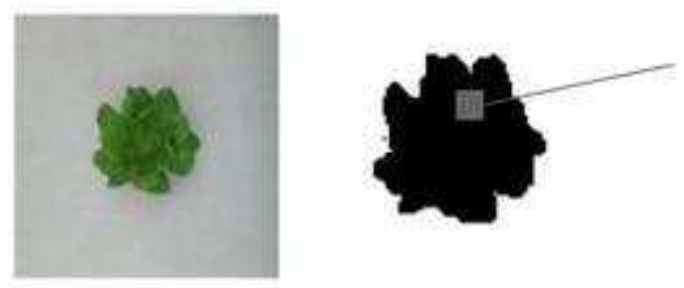

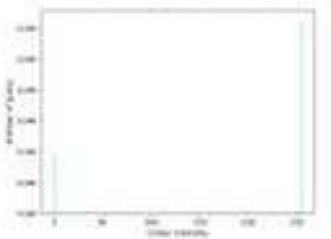

Thresholded Image and Histogram
Floodfill Algorithm

Binarize discrete regions
Final Image used to calculate LAI
Pixel by Pixel LAl calculation algorithm used.

Area in Pixels - 1919

LAl $-50.8535 \mathrm{~cm}^{\wedge} 2$

Per pixel area $=0.0265 \mathrm{~cm}^{\wedge} 2$
LAl used as label for Raw image ingested to $\mathrm{El}$ studio for model training

SONY IIIL EEDG IMPULSE

Figure 23

Pipeline created to estimate Ground Truth LAI 

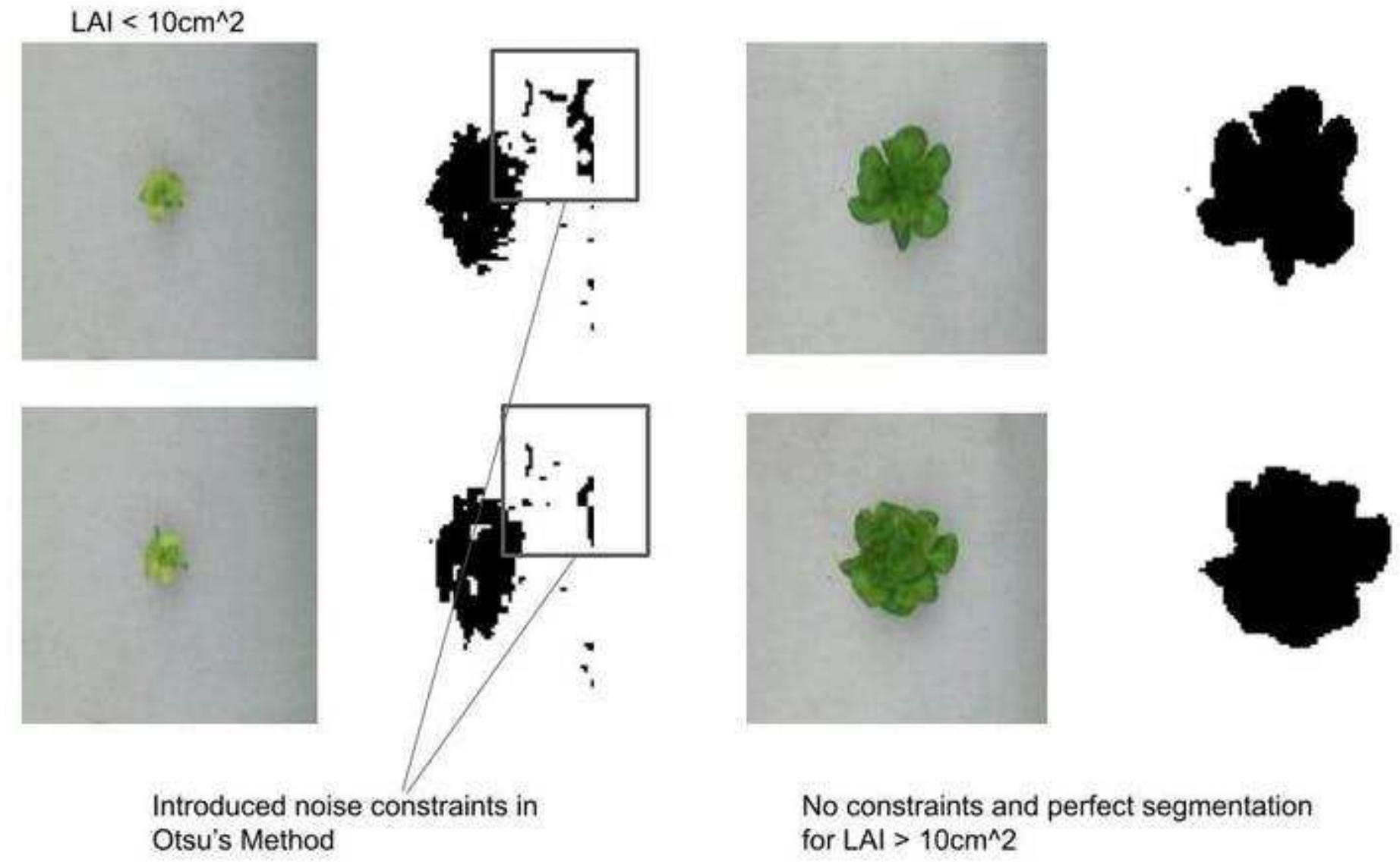

Figure 24

Comparing Otsu's threshold in Samples with $\mathrm{LAl}<$ or $>10 \mathrm{~cm} 2$

$$
\begin{aligned}
& \left.L A I_{(\text {perplant }}\right)=[[2 *(3.48 * 0.6 \text { metre }) / 100 \mathrm{n}] * \text { pixels }] \mathrm{cm}^{2} \\
& \mathrm{n}=\text { number of plants in cultivar } \\
& \text { pixels }=\text { Area in pixels }
\end{aligned}
$$

Figure 25

formulating LAI Index measure caclulation per pixel 


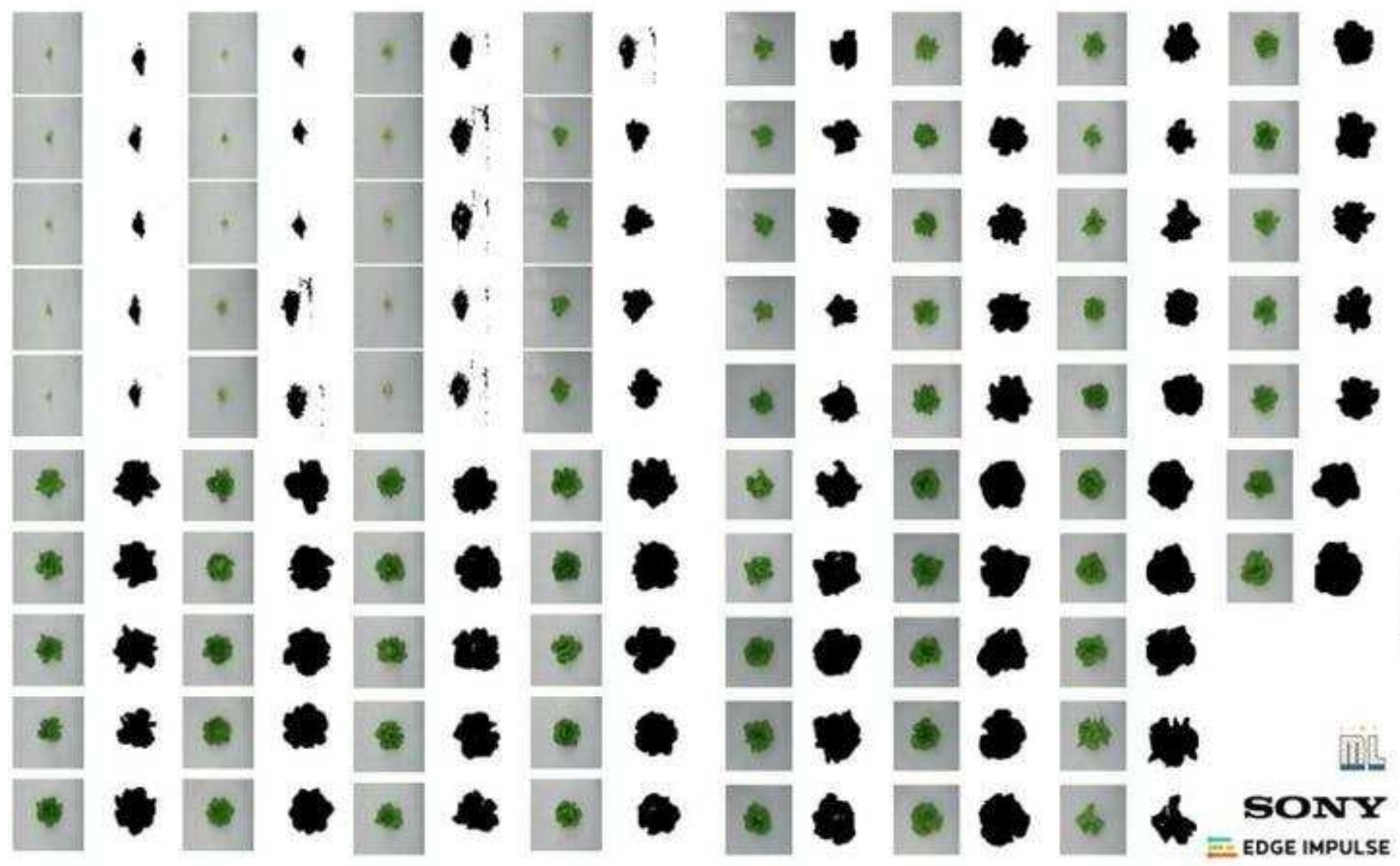

Figure 26

Adaptive Thresholding segmentation conducted on cultivar of 77 samples 

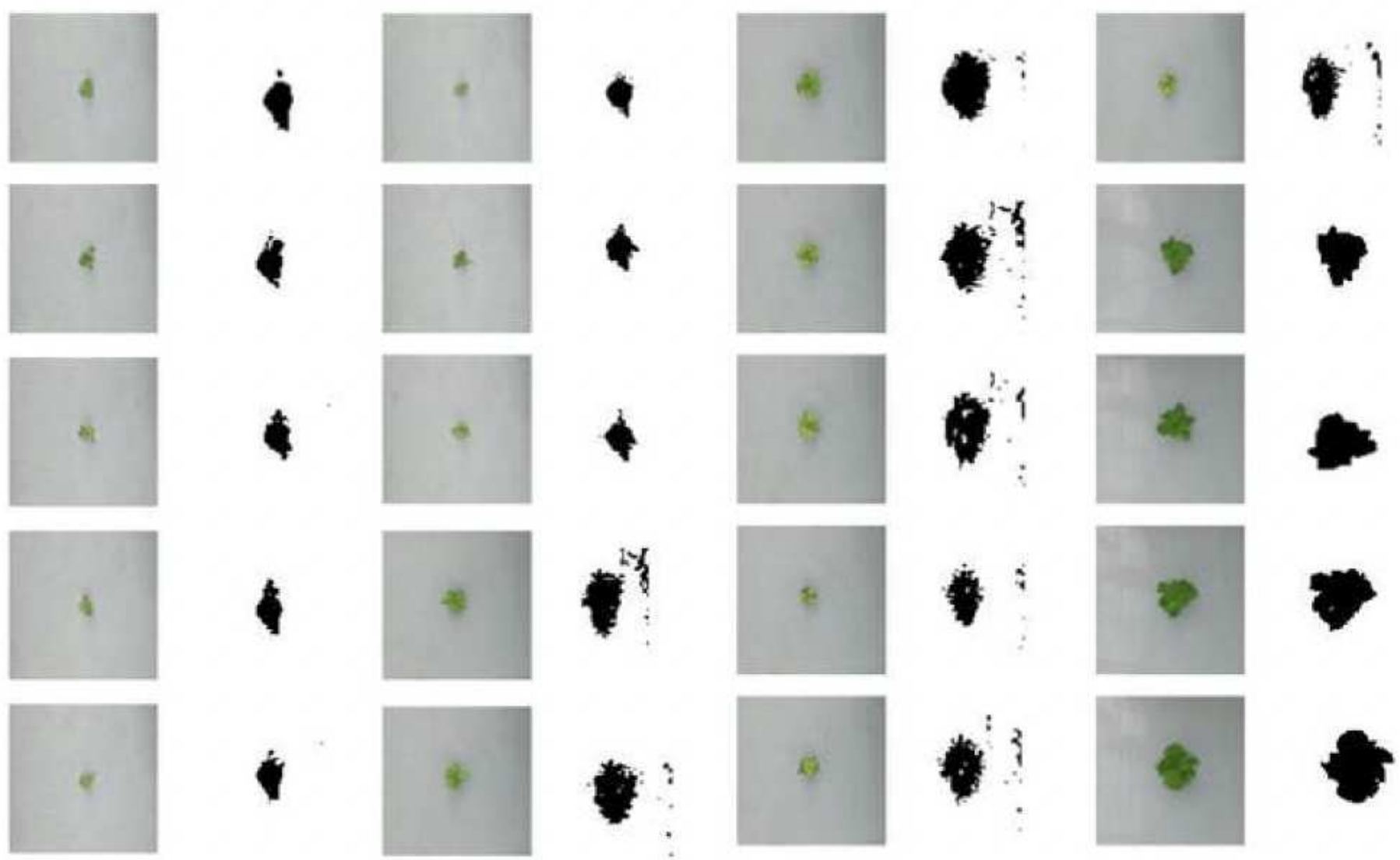

Figure 27

Thresholded Cultivars from Day 1 to 20 

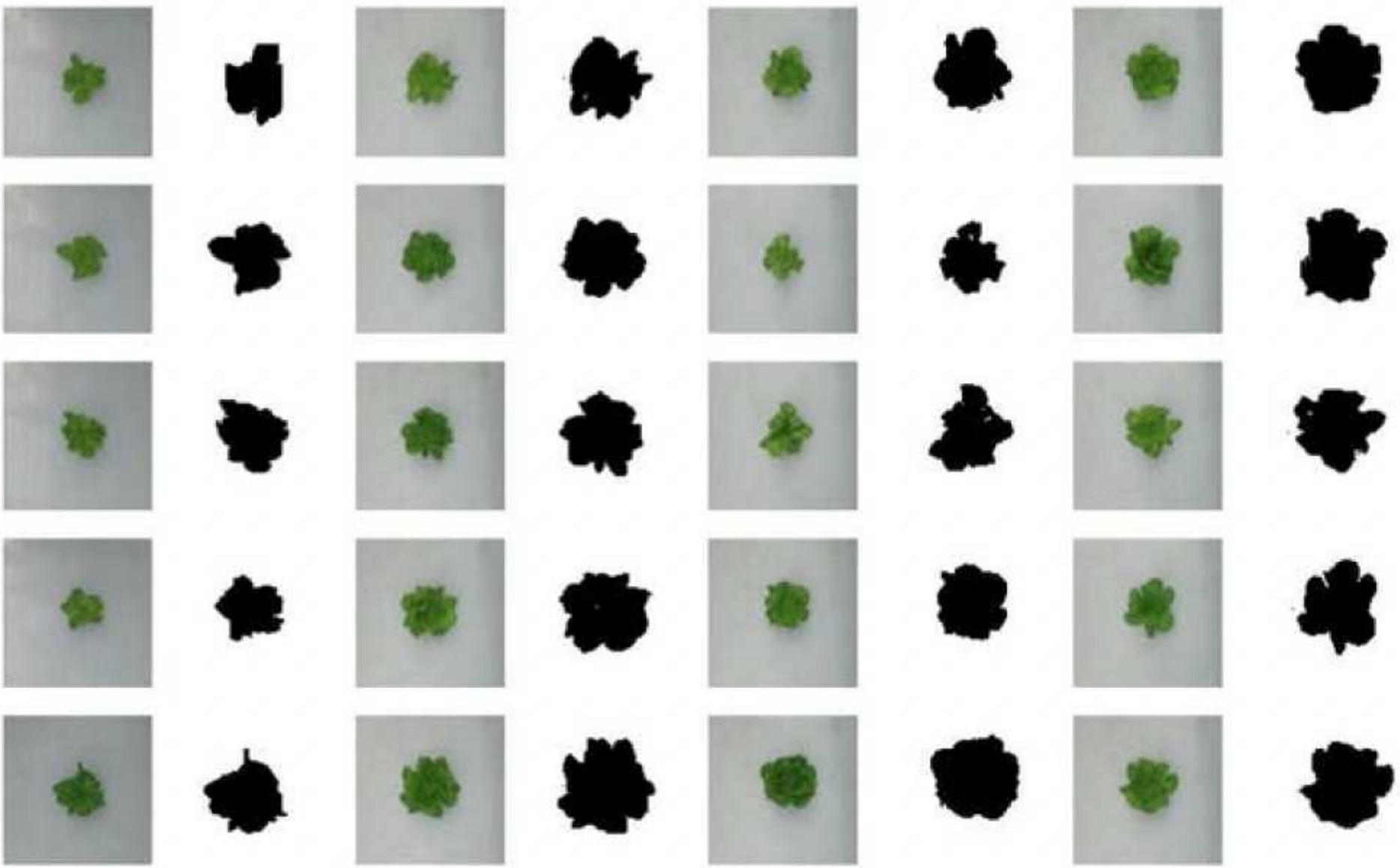

Figure 28

Thresholded Cultivars from Day 21 to 40 

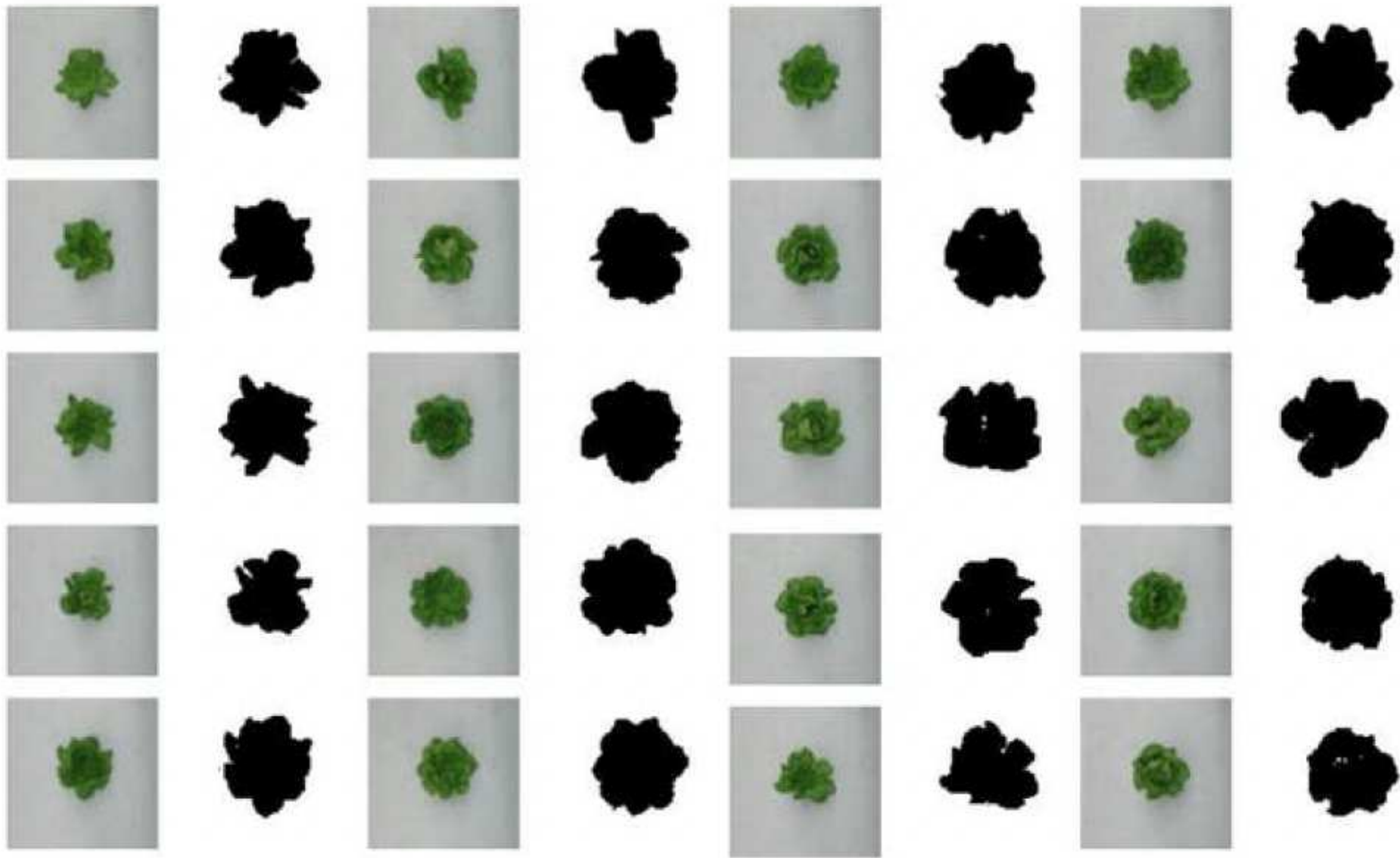

Figure 29

Thresholded Cultivars from Day 41 to 60 

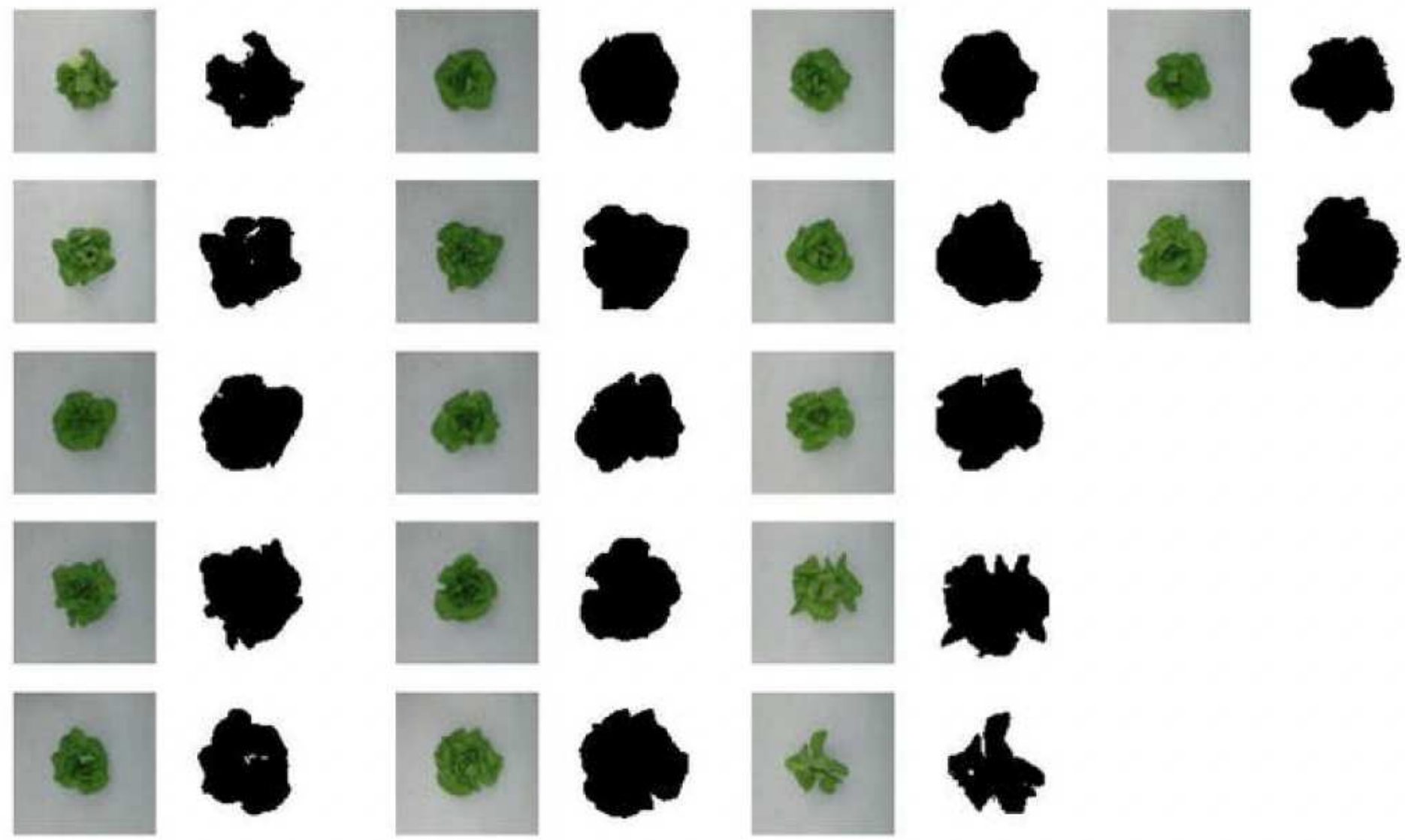

Figure 30

Thresholded Cultivars from Day 61 to 80

$$
\operatorname{RMSD}=\sqrt{\frac{\sum_{i=1}^{N}\left(x_{i}-\hat{x}_{i}\right)^{2}}{N}}
$$

$$
\begin{array}{ll}
\text { RMSD } & =\text { root-mean-square deviation } \\
i & =\text { variable } \mathrm{i} \\
N & =\text { number of non-missing data points } \\
x_{i} & =\text { actual observations time series } \\
\hat{x}_{i} & =\text { estimated time series }
\end{array}
$$

Figure 31

Formula used to calculate LAI RMSE on predicted outcome. 

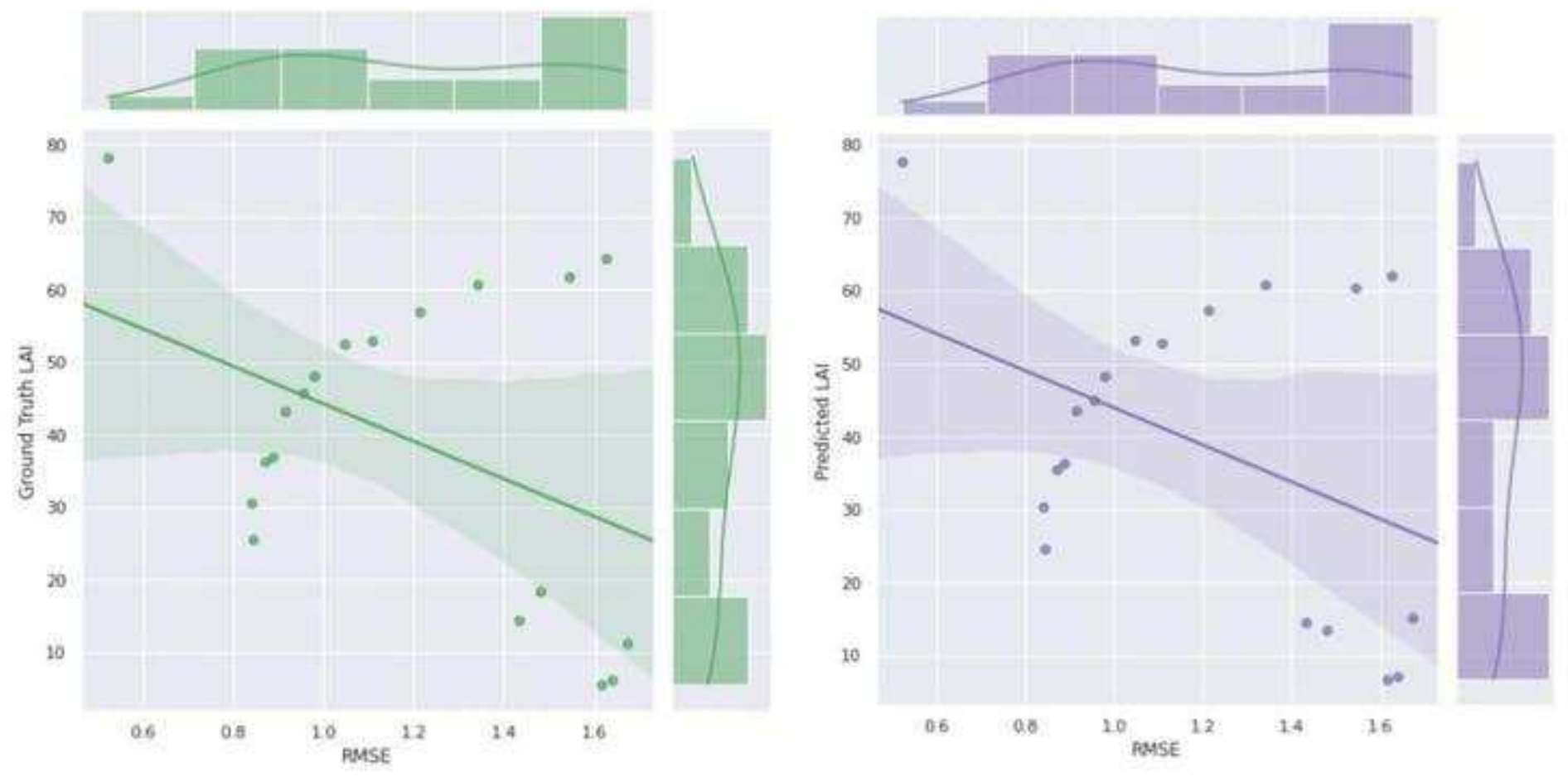

RMSE compared to Ground Truth LAI (Left) and Predicted LAI (Right)

\section{Figure 32}

RMSE Compared to Ground LAI (Left) and Predicted LAl (Right)

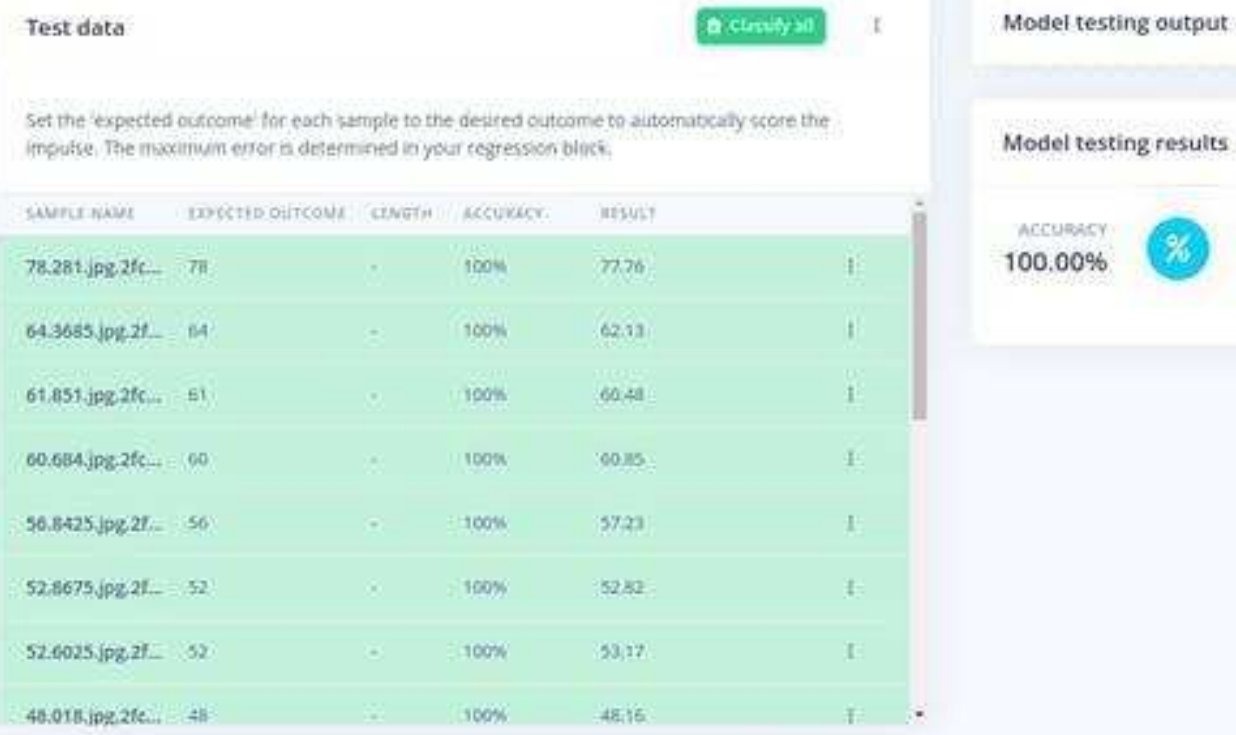

\section{Figure 33}

Prediction summary of over 19 samples in test dataset 


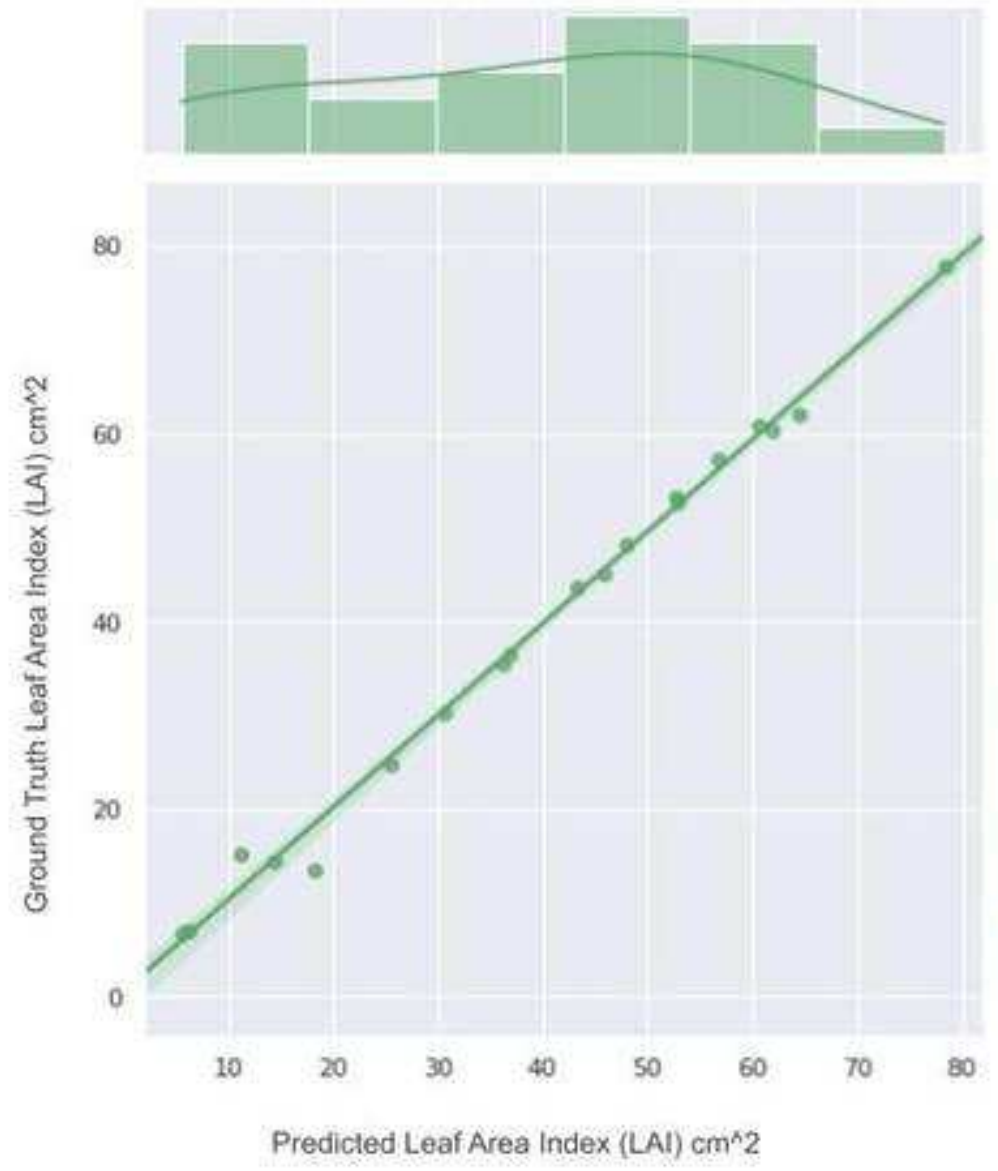
Total - 19 Samples of Lettuce Flandria used for Evaluation and Testing

Root Mean Squared Error:

RMSE $=1.1859 \mathrm{~cm}^{\wedge} 2$

Median Deviation between predicted and GT data:

(Deviation Index)

$\mathrm{DI}=-0.2351 \mathrm{~cm}^{\wedge} 2$

*Regression plot based on Data values predicted on Testing Tab in Edgelmpulse Studio

dhruvsheth.linkit@gmail.com

SONY HIL EDGE IMPULSE

\section{Figure 34}

Plot comparing Ground Truth and predicted LAl values from Edgelmpulse Studio Model Testing section 
Testing efficacy on Test Dataset
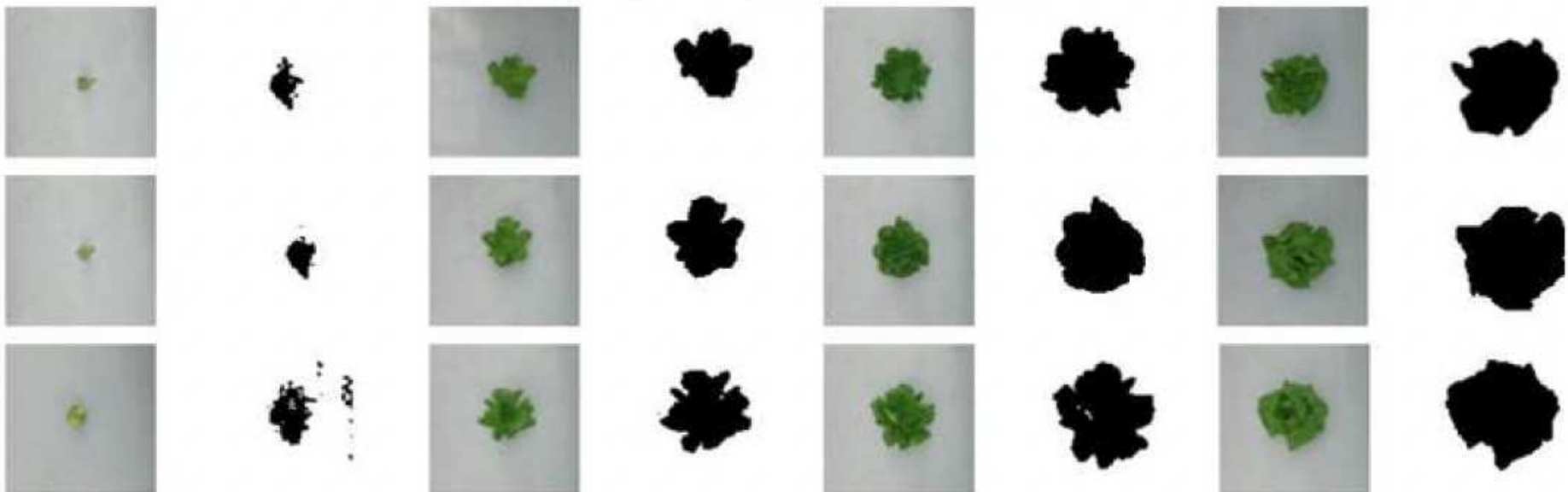

4
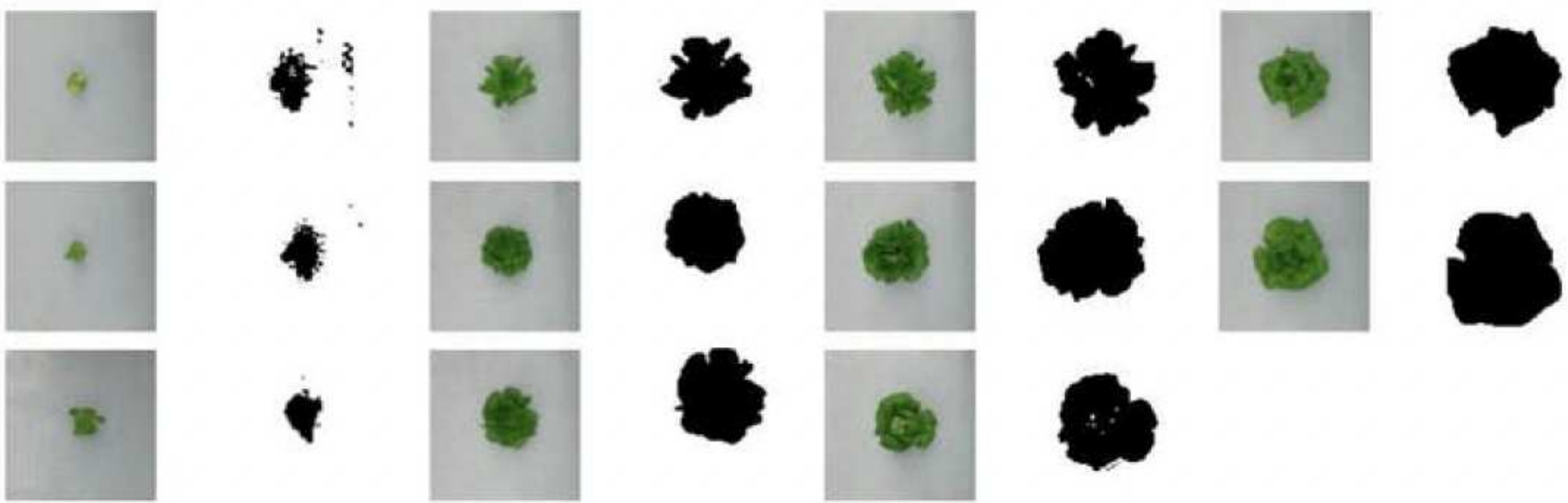

Figure 35

LAl of segmented images 


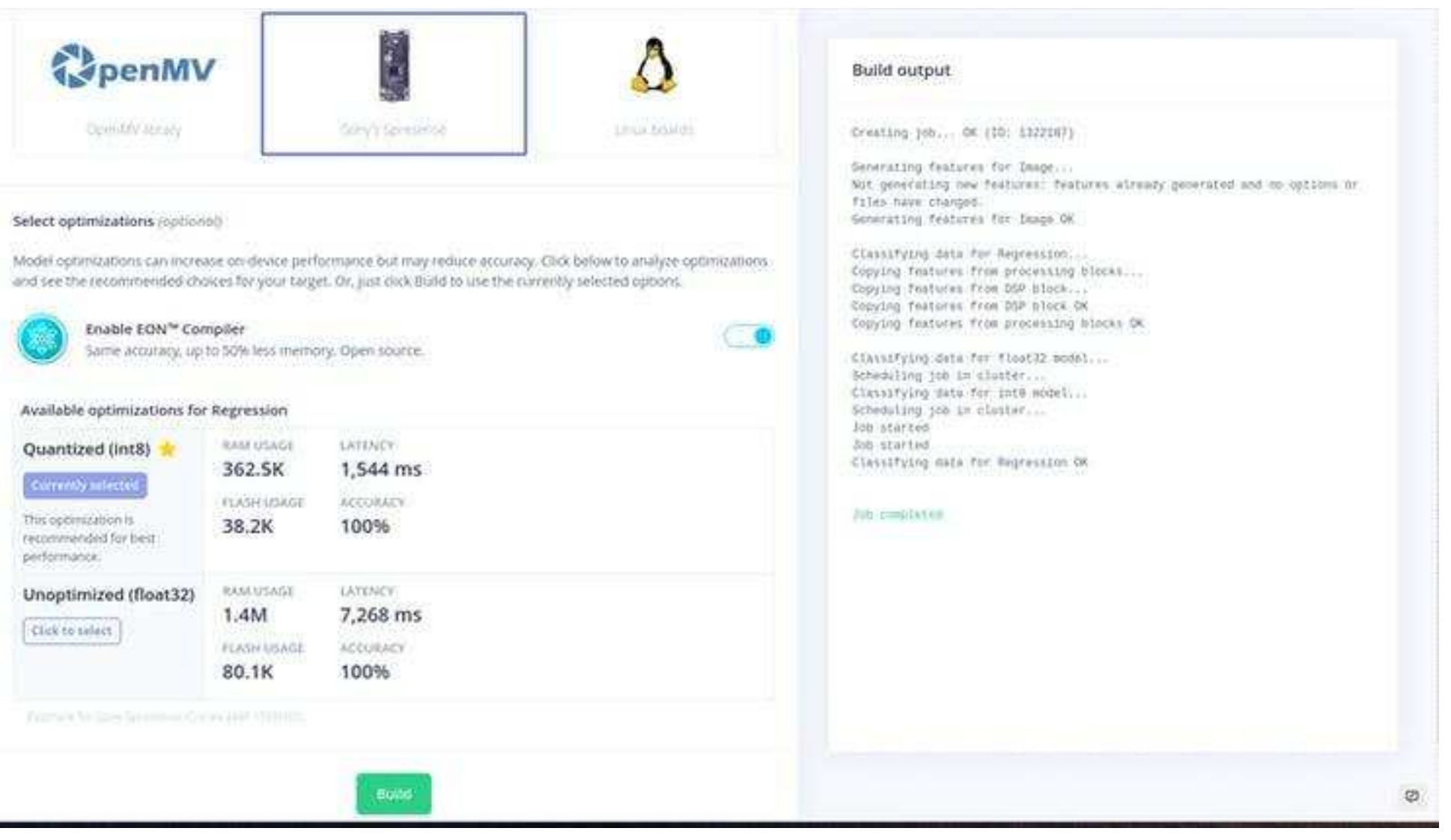

Figure 36

Compiled quantised binary for Regression model on Sony's Spresense 


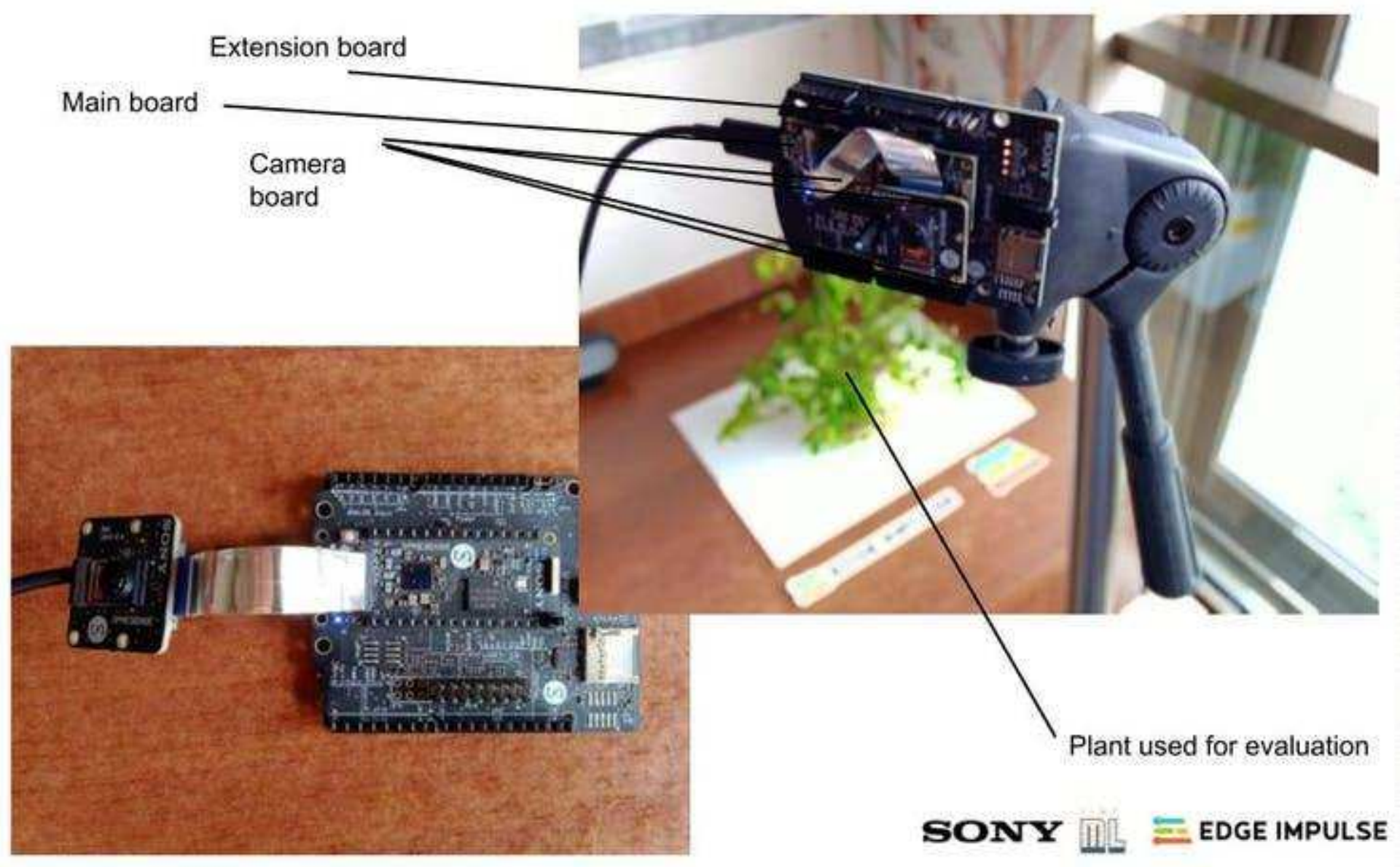

Figure 37

Demonstrating various components used in the hardware system for Test Data Acquisition 


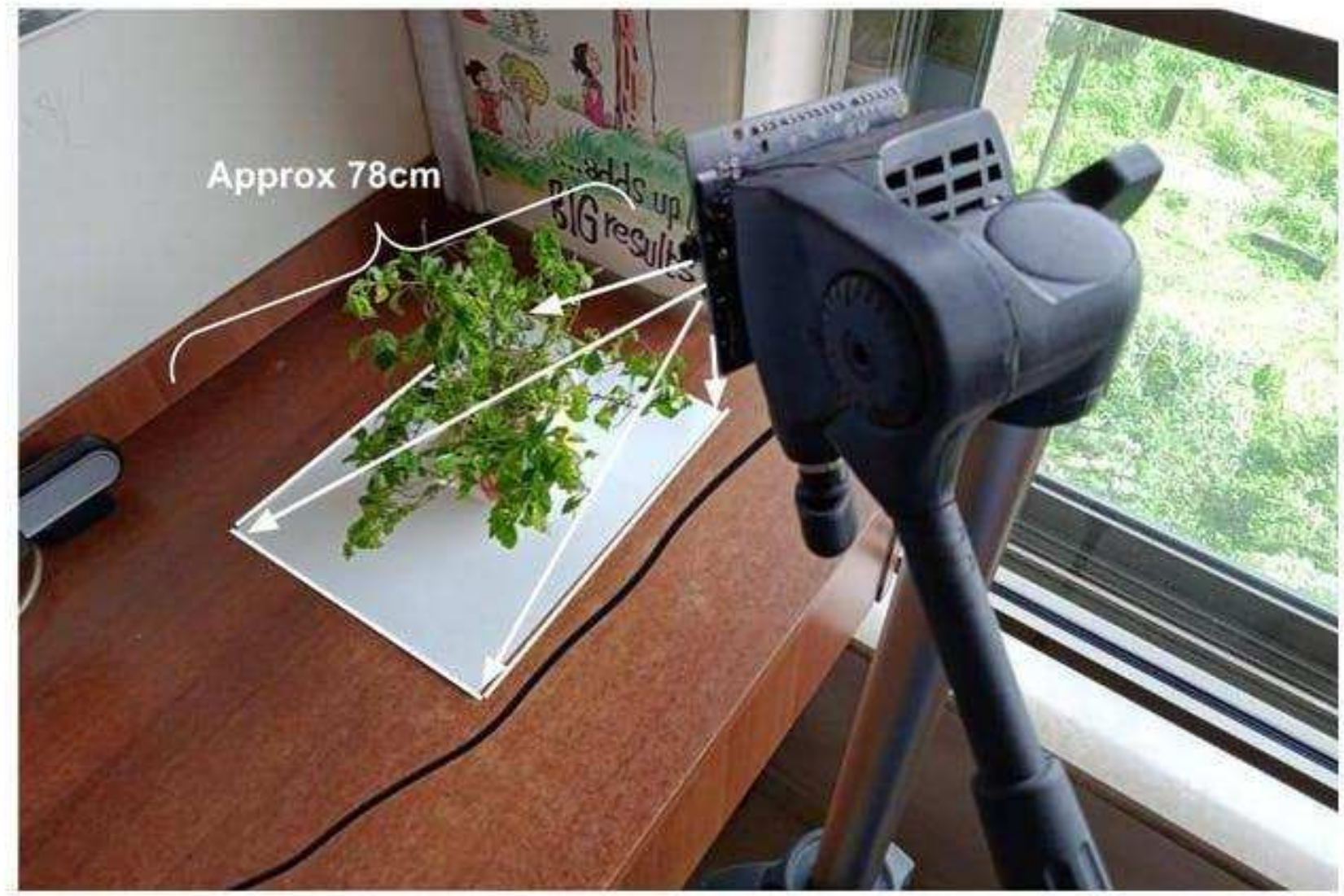

Figure 38

Demonstrates distance between Camera and Plant for the model to work 

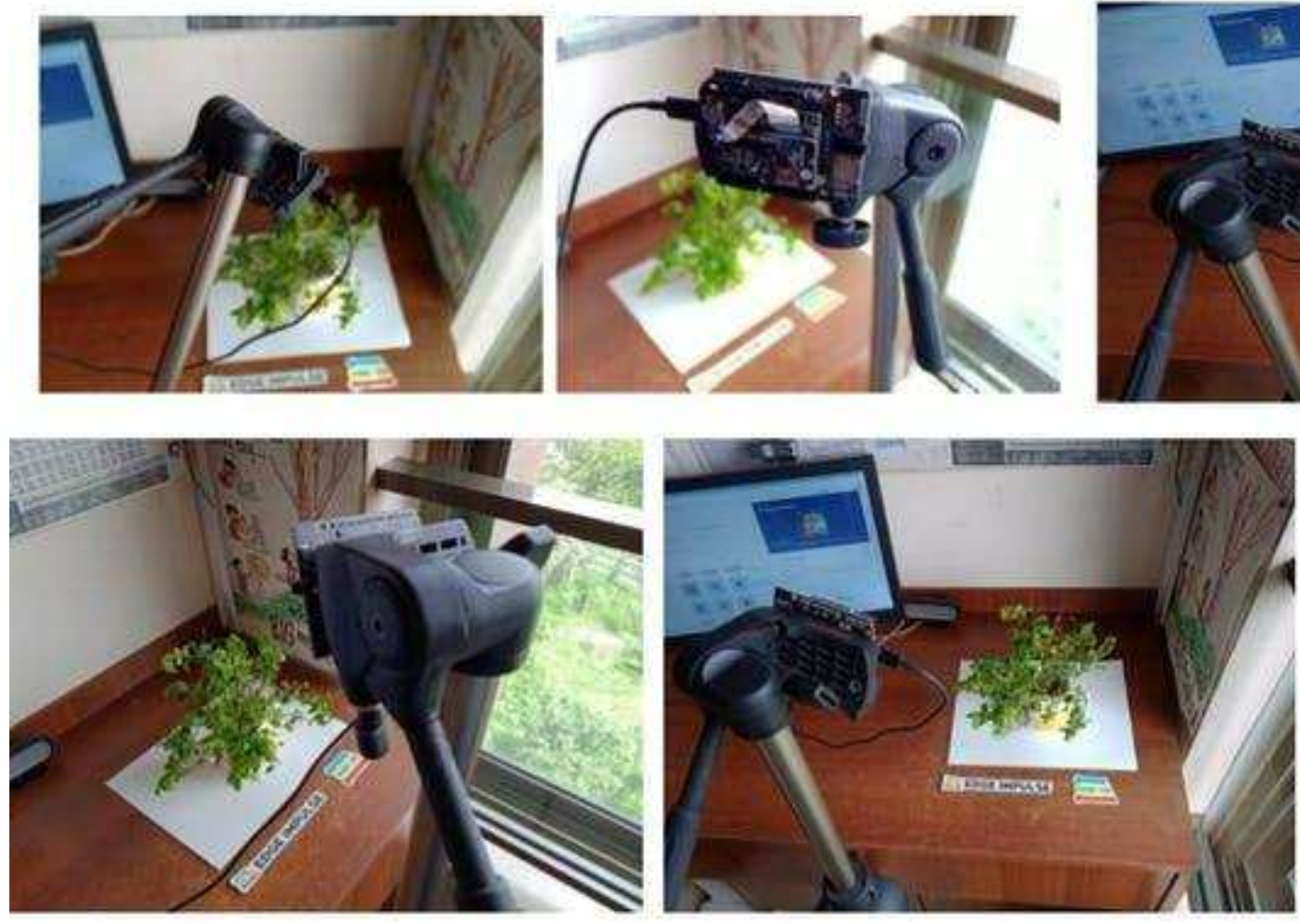

\section{Figure 39}

Various images of live classification and on-board processing algorithms

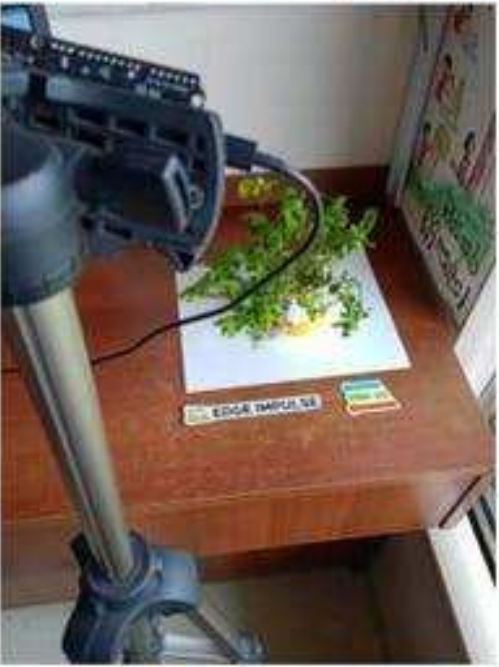




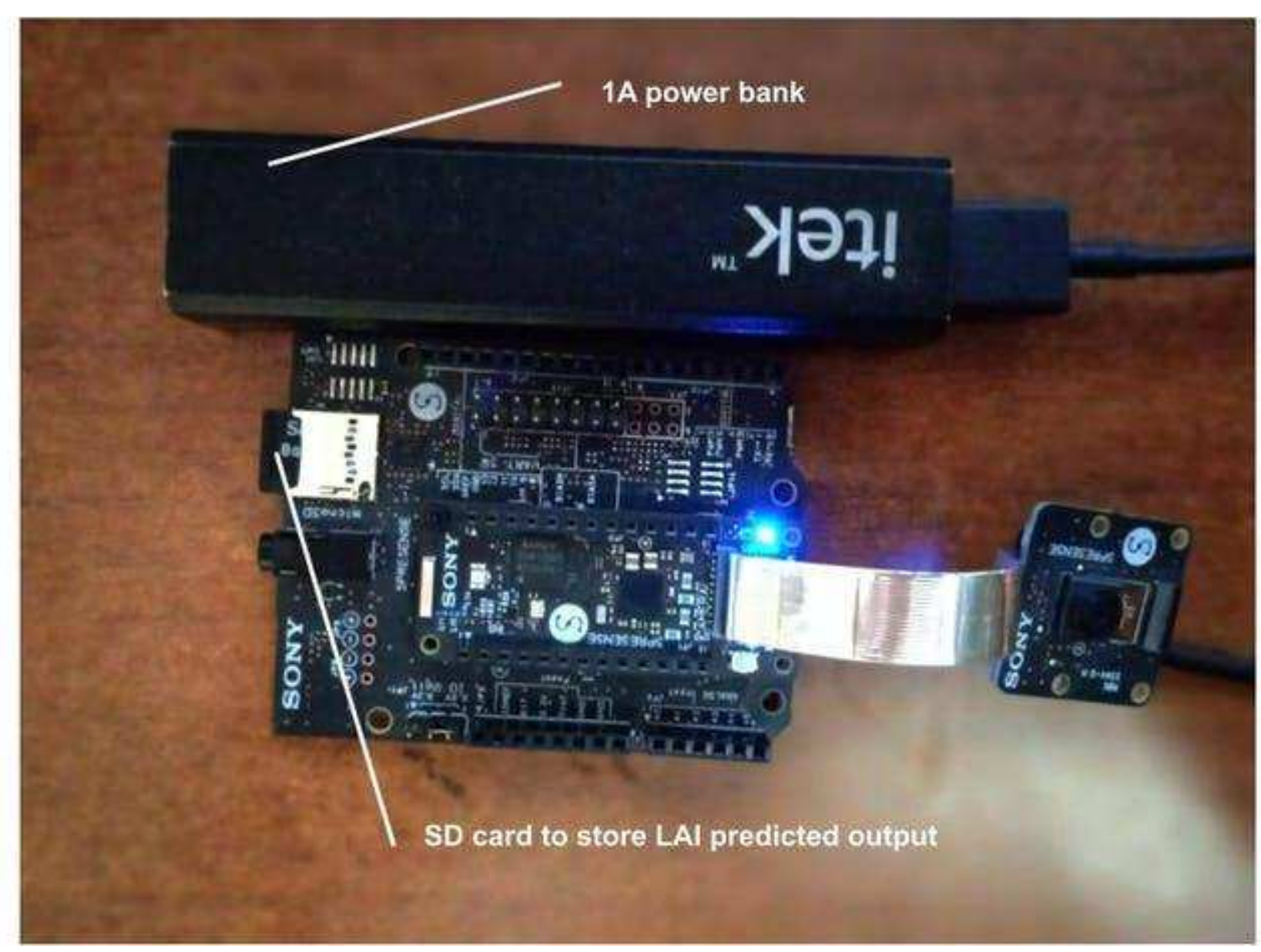

Figure 40

Battery Operated Telemetry system using SD card to store LAl predicted data 